\title{
A Versatile Route to Arylated Fluoroalkyl Bromide Building Blocks
}

\author{
Peter T. Kaplan and David A. Vicic*
}

Department of Chemistry, Lehigh University, 6 E Packer Ave., Bethlehem, PA 18015.

\section{1) Experimental Procedures:}

General Considerations: All manipulations were performed using standard Schlenk and high vacuum techniques or in a nitrogen filled glovebox. Solvents were purified by passing through activated alumina and/or copper in a solvent purification system supplied by Pure Process Technology. 1,4-

dibromooctafluorobutane and 1,6-dibromododecafluorohexane were purchased from SynQuest Labs, Inc. and used without further purification. Solution ${ }^{1} \mathrm{H}$ NMR spectra were recorded at ambient temperature on a Bruker DRX $500 \mathrm{MHz}$ spectrometer and referenced to residual proton solvent signals. ${ }^{19} \mathrm{~F}$ spectra were recorded on the Bruker NMR spectrometer operating at $470 \mathrm{MHz}$ and referenced to $\alpha, \alpha, \alpha$-trifluorotoluene as an internal standard $(\delta=-63.7)$. A Bruker D8 Quest diffractometer was used for X-ray crystal structure determinations. Mass spectral data were recorded on a HP 5890 Series II Plus GC/MS.

\section{Synthesis of 1-bromo-4-(trimethylsilyl)perfluorobutane (1):}

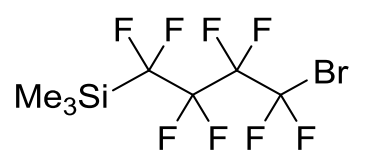

To a $100 \mathrm{~mL}$ round bottom flask $14.2864 \mathrm{~g}$ (39.7 mmol) of 1,4-dibromoperfluorobutane was added under $\mathrm{N}_{2}$ atmosphere followed by addition of $5.20 \mathrm{~mL}(41.0 \mathrm{mmol})$ of chlorotrimethylsilane and $34 \mathrm{~mL}$ of acetonitrile (MeCN). The solution was chilled at $-78{ }^{\circ} \mathrm{C}$ for 30 minutes, and then $11.2 \mathrm{~mL}(40.9 \mathrm{mmol}$ ) of tris(diethylamino)phosphine was slowly added. The mixture was stirred for $3.5 \mathrm{~h}$ at $-78^{\circ} \mathrm{C}$ and allowed to warm to room temperature. The mixture was evaporated under vacuum while heating the round bottom flask to $50{ }^{\circ} \mathrm{C}$ and collecting all volatile material in the cooled trap after about 1 $2 \mathrm{~h}$. The solution from the trap was collected and organic layer was extracted using pentane/water mixture in a separator funnel and dried over $\mathrm{Na}_{2} \mathrm{SO}_{4}$. Reactants/by-products and solvent were removed under vacuum $\left(30-34^{\circ} \mathrm{C}, 500\right.$ Torr for $15-$ $30 \mathrm{~min})$. Finally, the product was vacuum transferred over on the high vacuum line. The isolated yield of the colorless oil 1 was $(10.5 \mathrm{~g}, 75 \%) .{ }^{19} \mathrm{~F} \mathrm{NMR}\left(\mathrm{CDCl}_{3}, 470 \mathrm{MHz}\right): \delta-64.00(\mathrm{t}, J=13.7 \mathrm{~Hz}, 2 \mathrm{~F}),-118.13(\mathrm{t}, J=14.4 \mathrm{~Hz}, 2 \mathrm{~F}),-119.13(\mathrm{t}, J=13.6$ $\mathrm{Hz}, 2 \mathrm{~F}),-129.25$ (t, J=13.9 Hz, 2F). ${ }^{1} \mathrm{H}$ NMR (CDCl, $\left.300 \mathrm{MHz}\right): \delta 0.31(\mathrm{~s}, 9 \mathrm{H}) .{ }^{13} \mathrm{C} \mathrm{NMR}\left(\mathrm{CDCl}_{3}, 126 \mathrm{MHz}\right) \delta 125.48(\mathrm{tt}, J=$ 274.1, 44.7 Hz), 116.08 (tt, $J=314.4,38.2 \mathrm{~Hz}$ ), 109.98 (t quin, $J=267.4,34.0 \mathrm{~Hz}$ ), 113.33 (t quin, $J=260.1,31.2 \mathrm{~Hz}$ ). Anal. Calcd for $\mathrm{C}_{7} \mathrm{H}_{9} \mathrm{BrF}_{8} \mathrm{Si}$ : C, 23.81; $\mathrm{H}, 2.57$. Found: $\mathrm{C} 24.09 ; \mathrm{H} 2.64$. 


\section{Preparation of complex 2:}

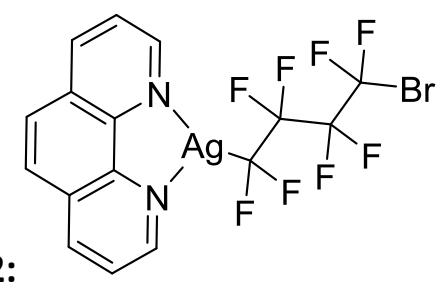

Addition of $0.3892 \mathrm{~g}(3.0678 \mathrm{mmol})$ of AgF was stirred in $5 \mathrm{~mL}$ of acetonitrile followed by $1.0520 \mathrm{~g}$ (2.9791 mmol) of 1 bromo-4-(trimethylsilyl)perfluorobutane. The mixture was stirred for $3.5 \mathrm{~h}$ and filtered through $0.2 \mu \mathrm{m}$ PTFE syringe filter giving a yellow solution. $0.5148 \mathrm{~g}(2.8567 \mathrm{mmol})$ 1,10-phenanthroline (phen) was dissolved into $1.00 \mathrm{~mL}$ of acetonitrile before being added to the stirring silver solution. After 10 minutes of stirring, a white solid precipitate is was visible. The solid was collected on a filter funnel and washed $3 x$ with $6 \mathrm{~mL}$ ether as the desired white crystalline product 2 in (1.04 g, $64 \%$ yield). Recrystallization of solid was possible using MeCN/ether to obtain colorless needles. ${ }^{19} \mathrm{~F} \mathrm{NMR}\left(\mathrm{THF}-d_{8}, 470\right.$ $\mathrm{MHz}$ ): $\delta-63.11(\mathrm{t}, J=11.7 \mathrm{~Hz}, 2 \mathrm{~F}),-106.77(\mathrm{~d}, J=53.8 \mathrm{~Hz}, 2 \mathrm{~F}),-116.98(\mathrm{t}, J=12.4 \mathrm{~Hz}, 2 \mathrm{~F}),-118.40(\mathrm{~s}, 2 \mathrm{~F}) .{ }^{1} \mathrm{H}$ NMR (THF- $d_{8}$, $300 \mathrm{MHz}$ ): $\delta 9.07(\mathrm{~d}, J=4.3 \mathrm{~Hz}, 2 \mathrm{H}), 8.57(\mathrm{~d}, J=8.2 \mathrm{~Hz}, 2 \mathrm{H}), 8.01(\mathrm{~s}, 2 \mathrm{H}), 7.90$ (dd, $J=8.1,4.6 \mathrm{~Hz}, 2 \mathrm{H})$. Anal. Calcd for $\mathrm{C}_{16} \mathrm{H}_{8} \mathrm{~F}_{8} \mathrm{~N}_{2} \mathrm{AgBr}$ : C, 33.83; $\mathrm{H}, 1.42$. Found: C 33.90; $\mathrm{H} 1.39$.

\section{Preparation of complex 6:}<smiles>FC(F)(Cl)C(F)(F)C(F)(F)C(F)(F)Br</smiles>

In a $20 \mathrm{~mL}$ vial $63.8 \mathrm{mg}(0.1123 \mathrm{mmol})$ of $\left(1,1,2,2,3,3,4,4\right.$-octafluoro-5-bromobutyl)(1,10-phenanthroline-k $\left.N^{1}, \mathrm{~K} N^{10}\right)$ silver reagent and $3 \mathrm{~mL}$ of $\mathrm{MeCN}$ were stirred for $1 \mathrm{~min} .31 .0 \mathrm{mg}(0.1628 \mathrm{mmol})$ Cul was added to the stirring mixture forming a red solution. The reaction was stirred for $20 \mathrm{~h}$ at $\mathrm{rt}$ followed by filtration of silver salt on a $0.2 \mu \mathrm{m}$ PTFE filter. $16 \mathrm{~mL}$ of ether was added to the dark red-orange solution and it was placed in the freezer for 7 days at $-37^{\circ} \mathrm{C}$. Orange needles formed in (39.4 mg, 67\%) yield and confirmed to be $\left.6 .{ }^{19} \mathrm{~F} \mathrm{NMR} \mathrm{(THF-} d_{8}, 470 \mathrm{MHz}\right): \delta-62.32(\mathrm{~s}, 2 \mathrm{~F}),-110.38(\mathrm{~s}, 2 \mathrm{~F}),-116.73$ $\left(\mathrm{t}, J_{\mathrm{FF}}=12.1 \mathrm{~Hz}, 2 \mathrm{~F}\right),-119.94\left(\mathrm{t}, J_{\mathrm{FF}}=13.0 \mathrm{~Hz} 2 \mathrm{~F}\right) .{ }^{1} \mathrm{H} \mathrm{NMR}\left(\mathrm{MeCN}-d_{3}, 300 \mathrm{MHz}\right): \delta 9.04(\mathrm{~s}, 2 \mathrm{H}), 8.69(\mathrm{~s}, 2 \mathrm{H}), 8.10(\mathrm{~s}, 2 \mathrm{H})$, 7.79 (s, 2H). Anal. Calcd for $\mathrm{C}_{16} \mathrm{H}_{8} \mathrm{~F}_{8} \mathrm{~N}_{2} \mathrm{Cu}$ : C, 36.70; H, 1.54. Found: C 36.59; $\mathrm{H} 1.47$. 


\section{Synthesis of 1-bromo-6-(trimethylsilyl)perfluorohexane (8):}

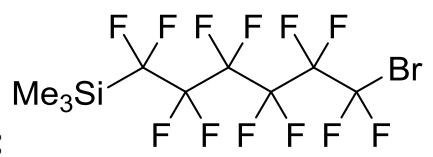

To a $20 \mathrm{~mL}$ vial $4.5992 \mathrm{~g}$ (10.001 mmol) of 1,6-dibromoperfluorohexane was added under $\mathrm{N}_{2}$ atmosphere followed by addition of $1.40 \mathrm{~mL}$ (11.031) of chlorotrimethylsilane and $10.0 \mathrm{~mL}$ of acetonitrile (MeCN). Chilled solution at $-78^{\circ} \mathrm{C}$ for 30 minutes, and then slowly add $2.80 \mathrm{~mL}\left(10.164 \mathrm{mmol}\right.$ ) of tris(diethylamino)phosphine and stir for $3.5 \mathrm{~h}$ at $-78{ }^{\circ} \mathrm{C}$ and allow it to warm to room temperature.. The mixture was evaporated under vacuum while heating the round bottom flask to 50 ${ }^{\circ} \mathrm{C}$ and collecting all volatile material in the cooled trap after about 1-2 $\mathrm{h}$. The solution from the trap was collected and organic layer was extracted using pentane/water mixture in a separator funnel and dried over $\mathrm{Na}_{2} \mathrm{SO}_{4}$. By-products and solvent were removed under vacuum ( $\mathrm{rt}, 500$ Torr for $5 \mathrm{~min}$ ). Vacuum distillation of solution at $50-60{ }^{\circ} \mathrm{C}$ (about 30 minutes of heating) produced colorless liquid 8 in $\left(1.40 \mathrm{~g}, 31 \%\right.$ yield). ${ }^{19} \mathrm{~F} \mathrm{NMR}\left(\mathrm{CDCl}_{3}, 470 \mathrm{MHz}\right): \delta-64.25(\mathrm{t}, J=14.4 \mathrm{~Hz}, 2 \mathrm{~F}),-118.25$ (s, 2F), -119.89 (s, 2F), -122.19 (s, 2F), -122.80 (s, 2F), -129.31 (t, J=14.4 Hz, 2F). ${ }^{1} \mathrm{H}$ NMR (CDCl, $\left.300 \mathrm{MHz}\right): \delta 0.30(\mathrm{~s}, 9 \mathrm{H})$. Anal. Calcd for $\mathrm{C}_{9} \mathrm{H}_{9} \mathrm{BrF}_{12} \mathrm{Si}$ : C, 23.86; H, 2.00. Found: $\mathrm{C} 22.86 ; \mathrm{H} 1.61$.

\section{Preparation of complex 9:}

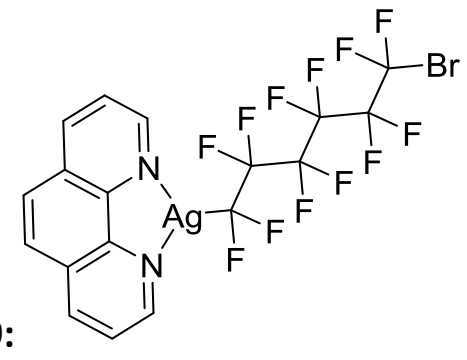

Addition of $194.8 \mathrm{mg}(1.425 \mathrm{mmol})$ of AgF was stirred with $4 \mathrm{~mL}$ of acetonitrile followed $645.9 \mathrm{mg}(1.425 \mathrm{mmol})$ of 1 bromo-6-(trimethylsilyl)perfluorohexane. The mixture was stirred for $3.5 \mathrm{~h}$ and filtered through $0.2 \mu \mathrm{m}$ PTFE syringe filter giving a yellow solution. $0.2555 \mathrm{~g}(1.4178 \mathrm{mmol})$ 1,10-phenanthroline (phen) was dissolved into $1.00 \mathrm{~mL}$ of acetonitrile before being added to the stirring silver solution. After 10 minutes of stirring, a white solid precipitate is was visible. The solid was collected on a filter funnel and washed $3 x$ with $4 \mathrm{~mL}$ ether as the desired off-white product 9 in $(437.9 \mathrm{mg}, 46 \%$ yield ${ }^{19} \mathrm{~F} \mathrm{NMR}\left(\mathrm{THF}-\mathrm{d}_{8}, 470 \mathrm{MHz}\right): \delta-65.38(\mathrm{t}, J=14.3 \mathrm{~Hz} 4 \mathrm{~F}),-108.86(\mathrm{~d}, J=52.4 \mathrm{~Hz} 2 \mathrm{~F}),-118.10(\mathrm{~s}, 2 \mathrm{~F}),-118.90(\mathrm{~s}, 2 \mathrm{~F}),-$ $121.62(\mathrm{~s}, 2 \mathrm{~F}),-121.84(\mathrm{~s}, 2 \mathrm{~F}) .{ }^{1} \mathrm{H}$ NMR (THF- $\left.d_{8}, 300 \mathrm{MHz}\right): \delta 9.02(\mathrm{~d}, J=3.4 \mathrm{~Hz}, 2 \mathrm{H}$ ), 8.51 (dd, $J=8.1,1.6 \mathrm{~Hz}, 2 \mathrm{H}), 7.97(\mathrm{~s}$, $2 \mathrm{H}$ ), 7.84 (dd, $J=8.1,4.5 \mathrm{~Hz}, 2 \mathrm{H}$ ). Anal. Calcd for $\mathrm{C}_{18} \mathrm{H}_{8} \mathrm{AgBrF}_{12} \mathrm{~N}_{2}: \mathrm{C}, 37.32 ; \mathrm{H}, 1.57$. Found: $\mathrm{C} 36.15 ; \mathrm{H} 2.66$.

\section{General Synthesis of $\mathrm{ArC}_{4} \mathrm{~F}_{8} \mathrm{Br}$ from Scheme 2:}

To a $20 \mathrm{~mL}$ vial, $60.0 \mathrm{mg}(0.106 \mathrm{mmol})$ of PhenAg( $\left(\mathrm{CF}_{2}\right)_{4} \mathrm{Br}$ was mixed in $1.0 \mathrm{~mL}$ of MeCN. Sequential addition of $0.100 \mathrm{mmol}$ of Ar-I and $20.0 \mathrm{mg}(0.105 \mathrm{mmol})$ of Cul were added to the vial. The solution changed from a white mixture to a reddish solution as solid precipitated to the bottom of the vial. After 1 day of stirring at room temperature, the solution was filtered through a $0.2 \mu \mathrm{m}$ PTFE filter and washed with an additional $0.5 \mathrm{~mL}$ of MeCN into a re-sealable Air tight NMR tube. Yields were calculated against $0.012 \mathrm{~mL}$ of $\alpha, \alpha, \alpha$-trifluorotoluene $(0.098 \mathrm{mmol})$ internal standard. Reactions not complete were heated at $50{ }^{\circ} \mathrm{C}$ overnight to give full conversion. 
$0.50 \mathrm{mmol}(0.10 \mathrm{mmol}$ excess of silver reagent and copper salt) reactions ran in $6.0 \mathrm{~mL}$ of MeCN with $5.0 \mathrm{~mL}$ of MeCN to transfer. Filtration was accomplished using fine frit glass filter funnels and re-sealable air tight ampules.

A gram scale reaction $(5.0 \mathrm{mmol}, 0.1 \mathrm{mmol}$ excess of silver reagent and copper salt) reaction ran in $33.0 \mathrm{~mL}$ of $\mathrm{MeCN}$ in a $100 \mathrm{~mL}$ RBF with $5.0 \mathrm{~mL}$ of MeCN to transfer. Filtration was accomplished using fine frit glass filter funnels. $58 \%$ of product was collected.

Isolation was accomplished by removing solvent under reduced pressure followed by column chromatography using hexanes, hexanes/DCM $50 \%$, or DCM.

\section{General Synthesis of $\mathrm{ArC}_{6} \mathrm{~F}_{12} \mathrm{Br}$ from Scheme 2:}

To a $20 \mathrm{~mL}$ vial, $60.0 \mathrm{mg}$ (0.106 mmol) of PhenAg( $\left(\mathrm{CF}_{2}\right)_{4} \mathrm{Br}$ was mixed in $1.0 \mathrm{~mL}$ of MeCN. Sequential addition of $0.100 \mathrm{mmol}$ of Ar-I and $20.0 \mathrm{mg}(0.105 \mathrm{mmol})$ of Cul were added to the vial. The solution changed from a white mixture to a reddish solution as solid precipitated to the bottom of the vial. After 1 day of stirring at room temperature, the solution was filtered through a $0.2 \mu \mathrm{m}$ PTFE filter and washed with an additional $0.5 \mathrm{~mL}$ of MeCN into a re-sealable Air tight NMR tube. Yields were calculated against $0.012 \mathrm{~mL}$ of $\alpha, \alpha, \alpha$-trifluorotoluene $(0.098 \mathrm{mmol})$ internal standard. Reactions not complete were heated at $50{ }^{\circ} \mathrm{C}$ overnight to give full conversion Isolation was accomplished by removing solvent under reduced pressure followed by column chromatography using hexanes, hexanes/DCM $50 \%$, or DCM.

\section{2-(4-bromo-1,1,2,2,3,3,4,4-octafluorobutyl)pyridine}

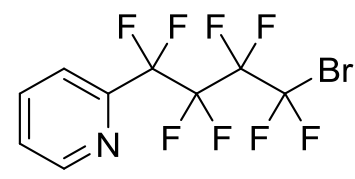

Synthesised following general procedure scheme 2 from 2 -iodopyridine $(0.50 \mathrm{mmol}$ scale). Purification by flash silica column chromatography (eluent: $50 \%$ dichloromethane in hexanes) to yield $93 \%$ by internal standard, $\alpha, \alpha, \alpha-$ trifluorotoluene $(84.1 \mathrm{mg}, 47 \%$ yield isolated yield) as a light yellow oil. Analytical data for 2-(4-bromo-1,1,2,2,3,3,4,4octafluorobutyl)pyridine (7a) ${ }^{19} \mathrm{~F} \mathrm{NMR}\left(\mathrm{CDCl}_{3}, 470 \mathrm{MHz}\right): \delta-64.08(\mathrm{t}, J=13.7 \mathrm{~Hz}, 2 \mathrm{~F}),-115.12(\mathrm{t}, J=13.1 \mathrm{~Hz}, 2 \mathrm{~F}),-117.73$ $(\mathrm{t}, J=12.8 \mathrm{~Hz}, 2 \mathrm{~F}),-122.09(\mathrm{t}, J=12.9 \mathrm{~Hz}, 2 \mathrm{~F}) .{ }^{1} \mathrm{H} \mathrm{NMR}\left(\mathrm{CDCl}_{3}, 300 \mathrm{MHz}\right): \delta 8.78(\mathrm{~d}, J=4.7 \mathrm{~Hz}, 1 \mathrm{H}), 7.89(\mathrm{td}, J=7.8,1.7 \mathrm{~Hz}$, $1 \mathrm{H}), 7.70(\mathrm{~d}, J=7.9 \mathrm{~Hz}, 1 \mathrm{H}), 7.75(\mathrm{dd}, J=7.7,5.2 \mathrm{~Hz}, 1 \mathrm{H})$. Exact mass (El+) calcd for $\mathrm{C}_{9} \mathrm{H}_{4} \mathrm{NF}_{8} \mathrm{Br} 356.9399$, found 356.9427.

\section{3-(4-bromo-1,1,2,2,3,3,4,4-octafluorobutyl)pyridine}

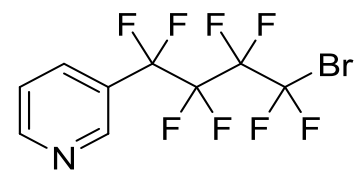

Synthesised following general procedure scheme 2 from 3-iodopyridine $(0.10 \mathrm{mmol}$ scale) to yield $>99 \%$ by internal standard, $\alpha, \alpha, \alpha$-trifluorotoluene as a light yellow oil. Analytical data for 3-(4-bromo-1,1,2,2,3,3,4,4octafluorobutyl)pyridine (7b) ${ }^{19} \mathrm{~F} \mathrm{NMR}\left(\mathrm{MeCN}^{-} \mathrm{d}_{3}, 470 \mathrm{MHz}\right.$ ): $\delta \quad-65.88$ (s, 2F), -112.38 (s, 2F), -117.82 (s, 2F), -122.17 (s, 2F). ${ }^{1} \mathrm{H}$ NMR $\left(\mathrm{MeCN}-d_{3}, 300 \mathrm{MHz}\right): \delta 8.70-8.35(\mathrm{~m}, 2 \mathrm{H}), 8.02(\mathrm{~d}, J=8.1 \mathrm{~Hz}, 1 \mathrm{H}), 7.57(\mathrm{dd}, \mathrm{J}=6.0,1.6 \mathrm{~Hz}, 1 \mathrm{H})$. Exact mass (EI+) calcd for $\mathrm{C}_{9} \mathrm{H}_{4} \mathrm{NF}_{8} \mathrm{Br} 356.9399$, found 356.9400 . 


\section{2-(4-bromo-1,1,2,2,3,3,4,4-octafluorobutyl)thiophene}

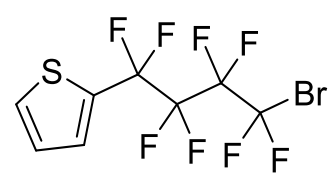

Synthesised following general procedure scheme 2 from 2 -iodothiophene $(0.10 \mathrm{mmol}$ scale $)$ to yield $>99 \%$ by internal standard, $\alpha, \alpha, \alpha$-trifluorotoluene as a colorless oil. Analytical data for 2-(4-bromo-1,1,2,2,3,3,4,4octafluorobutyl)thiophene (7c) ${ }^{19} \mathrm{~F} \mathrm{NMR}\left(\mathrm{MeCN}-d_{3}, 470 \mathrm{MHz}\right): \delta \quad-65.82(\mathrm{t}, J=14.3 \mathrm{~Hz}, 2 \mathrm{~F}),-101.57(\mathrm{t}, J=14.0 \mathrm{~Hz}, 2 \mathrm{~F})$, $117.86(\mathrm{~s}, 2 \mathrm{~F}),-121.90(\mathrm{~s}, 2 \mathrm{~F}) .{ }^{1} \mathrm{H}$ NMR $\left(\mathrm{MeCN}_{-} d_{3}, 300 \mathrm{MHz}\right.$ ): $\delta 7.80(\mathrm{dd}, J=5.0,1.3 \mathrm{~Hz}, 1 \mathrm{H}), 7.57(\mathrm{~d}, J=3.7 \mathrm{~Hz}, 1 \mathrm{H}), 7.26-$ $7.20(\mathrm{~m}, 1 \mathrm{H})$. Exact mass $(\mathrm{El}+)$ calcd for $\mathrm{C}_{8} \mathrm{H}_{3} \mathrm{SF}_{8} \mathrm{Br} 361.9011$, found 361.9008 .

\section{3-(4-bromo-1,1,2,2,3,3,4,4-octafluorobutyl)thiophene}

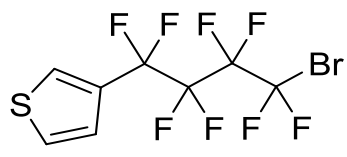

Synthesised following general procedure scheme 2 from 3-iodothiophene $(0.10 \mathrm{mmol}$ scale) to yield $97 \%$ by internal standard, $\alpha, \alpha, \alpha$-trifluorotoluene as a colorless oil. Analytical data for 3-(4-bromo-1,1,2,2,3,3,4,4octafluorobutyl)thiophene (7d) ${ }^{19} \mathrm{~F} \mathrm{NMR}\left(\mathrm{MeCN}-d_{3}, 470 \mathrm{MHz}\right): \delta \quad-65.76(\mathrm{t}, J=13.7 \mathrm{~Hz}, 2 \mathrm{~F}),-106.81(\mathrm{t}, J=13.3 \mathrm{~Hz}, 2 \mathrm{~F}),-$ $117.99(\mathrm{t}, J=13.8 \mathrm{~Hz}, 2 \mathrm{~F}),-122.47(\mathrm{~s}, 2 \mathrm{~F}) .{ }^{1} \mathrm{H}$ NMR $\left(\mathrm{MeCN}-d_{3}, 300 \mathrm{MHz}\right): \delta 7.55$ (dd, $\left.J=3.0,1.1 \mathrm{~Hz}, 1 \mathrm{H}\right), 7.35(\mathrm{dd}, J=5.0$, $3.0 \mathrm{~Hz}, 1 \mathrm{H}), 7.14(\mathrm{dd}, J=5.0,1.1 \mathrm{~Hz}, 1 \mathrm{H})$. Exact mass (El+) calcd for $\mathrm{C}_{8} \mathrm{H}_{3} \mathrm{SF}_{8} \mathrm{Br}$ 361.9011, found 361.9007 .

\section{7-chloro-4-(4-bromo-1,1,2,2,3,3,4,4-octafluorobutyl)quinoline}

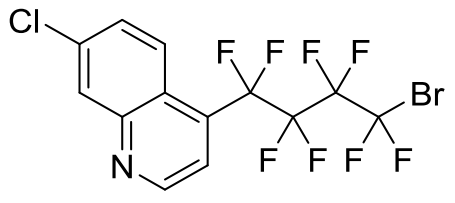

Synthesised following general procedure scheme 2 from 7-chloro-4-iodoquinoline $(0.10 \mathrm{mmol}$ scale). Purification by flash silica column chromatography (eluent: dichloromethane) to yield $93 \%$ by internal standard, $\alpha, \alpha, \alpha$-trifluorotoluene (32.3 $\mathrm{mg}$, 73\% yield isolated yield) as a white solid. Analytical data for 7-chloro-4-(4-bromo-1,1,2,2,3,3,4,4octafluorobutyl)quinoline (7e) ${ }^{19} \mathrm{~F} \mathrm{NMR}\left(\mathrm{CDCl}_{3}, 470 \mathrm{MHz}\right): \delta-64.24(\mathrm{t}, J=14.9 \mathrm{~Hz}, 2 \mathrm{~F}),-107.86(\mathrm{t}, J=14.3 \mathrm{~Hz}, 2 \mathrm{~F}),-117.61$ $(\mathrm{t}, J=14.2 \mathrm{~Hz}, 2 \mathrm{~F}),-112.17(\mathrm{t}, J=14.2 \mathrm{~Hz}, 2 \mathrm{~F}) .{ }^{1} \mathrm{H} \mathrm{NMR}\left(\mathrm{CDCl}_{3}, 300 \mathrm{MHz}\right): \delta 9.08(\mathrm{~s}, 1 \mathrm{H}), 8.23(\mathrm{~s}, 1 \mathrm{H}), 8.13(\mathrm{~s}, 1 \mathrm{H}), 7.66(\mathrm{~s}$, $2 \mathrm{H})$. Exact mass (El+) calcd for $\mathrm{C}_{13} \mathrm{H}_{5} \mathrm{NF}_{8} \mathrm{ClBr} 440.9166$, found 440.9166

\section{1-(4-bromo-1,1,2,2,3,3,4,4-octafluorobutyl)benzene}

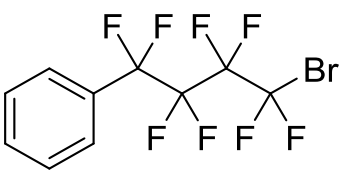

Synthesised following general procedure scheme 2 from iodobenzene $(0.10 \mathrm{mmol}$ scale) to yield $88 \%$ by internal standard, $\alpha, \alpha, \alpha$-trifluorotoluene as a colorless oil. Analytical data for 1-(4-bromo-1,1,2,2,3,3,4,4-octafluorobutyl)benzene (7f) ${ }^{19} \mathrm{~F}$ NMR ((MeCN- $\left.d_{3}, 470 \mathrm{MHz}\right): \delta-65.73(\mathrm{~s}, 2 \mathrm{~F}),-111.54(\mathrm{~s}, 2 \mathrm{~F}),-117.84(\mathrm{~s}, 2 \mathrm{~F}),-122.14(\mathrm{~s}, 2 \mathrm{~F}) .{ }^{1} \mathrm{H}$ NMR $\left(\mathrm{MeCN}-d_{3}, 300 \mathrm{MHz}\right)$ : $\delta 7.66(d, J=8.1 \mathrm{~Hz}, 2 \mathrm{H}), 7.59(\mathrm{~d}, J=8.1 \mathrm{~Hz}, 2 \mathrm{H})$. Exact mass $(\mathrm{El}+)$ calcd for $\mathrm{C}_{10} \mathrm{H}_{5} \mathrm{~F}_{8} \mathrm{Br} 355.9447$, found 355.9465. 


\section{4-(4-bromo-1,1,2,2,3,3,4,4-octafluorobutyl)biphenyl}

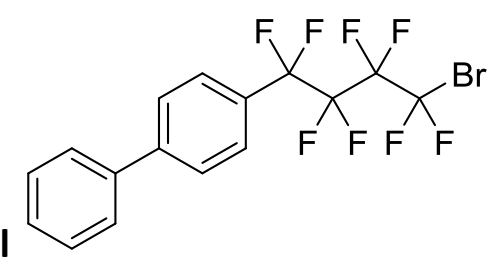

Synthesised following general procedure scheme 2 from 4 -iodobiphenyl ( $0.50 \mathrm{mmol}$ scale). Purification by flash silica column chromatography (eluent: hexanes) to yield $90 \%$ by internal standard, $\alpha, \alpha, \alpha$-trifluorotoluene $(153.8 \mathrm{mg}, 71 \%$ yield isolated yield) as a white solid. Analytical data for 4-(4-bromo-1,1,2,2,3,3,4,4-octafluorobutyl)biphenyl (7g) ${ }^{19} \mathrm{~F} \mathrm{NMR}$ $\left(\mathrm{CDCl}_{3}, 470 \mathrm{MHz}\right): \delta-64.03(\mathrm{t}, J=14.2 \mathrm{~Hz}, 2 \mathrm{~F}),-111.59(\mathrm{t}, J=14.6 \mathrm{~Hz}, 2 \mathrm{~F}),-117.59(\mathrm{t}, J=15.0 \mathrm{~Hz}, 2 \mathrm{~F}),-121.98(\mathrm{t}, J=12.9 \mathrm{~Hz}$, 2F). ${ }^{1} \mathrm{H} \mathrm{NMR}\left(\mathrm{CDCl}_{3}, 300 \mathrm{MHz}\right): \delta 7.71(\mathrm{~d}, J=8.6 \mathrm{~Hz}, 2 \mathrm{H}), 7.67(\mathrm{~d}, J=7.9 \mathrm{~Hz}, 2 \mathrm{H}), 7.63(\mathrm{~d}, J=8.6 \mathrm{~Hz}, 2 \mathrm{H}), 7.48(\mathrm{t}, J=7.8 \mathrm{~Hz}$, $2 \mathrm{H}), 7.42(\mathrm{tt}, J=8.4,1.0 \mathrm{~Hz}, 1 \mathrm{H})$. Exact mass $(\mathrm{El}+)$ calcd for $\mathrm{C}_{16} \mathrm{H}_{9} \mathrm{~F}_{8} \mathrm{Br} 431.9760$, found 431.9767 .

\section{2-(4-bromo-1,1,2,2,3,3,4,4-octafluorobutyl)naphthalene}<smiles>FC(F)(Br)C(F)(F)C(F)(F)C(F)(F)c1ccc2ccccc2c1</smiles>

Synthesised following general procedure scheme 2 from 2 -iodonaphthalene $(0.10 \mathrm{mmol}$ scale $)$ to yield $>99 \%$ by internal standard, $\alpha, \alpha, \alpha$-trifluorotoluene as a white solid. Analytical data for 2-(4-bromo-1,1,2,2,3,3,4,4octafluorobutyl)naphthalene (7h) ${ }^{19} \mathrm{~F} \mathrm{NMR}\left(\mathrm{CDCl}_{3}, 470 \mathrm{MHz}\right): \delta-64.03(\mathrm{t}, J=13.3 \mathrm{~Hz}, 2 \mathrm{~F}),-111.18(\mathrm{t}, J=13.3 \mathrm{~Hz}, 2 \mathrm{~F})$, $117.55(\mathrm{t}, J=16.0 \mathrm{~Hz}, 2 \mathrm{~F}),-121.80(\mathrm{t}, J=13.3 \mathrm{~Hz}, 2 \mathrm{~F}) .{ }^{1} \mathrm{H} \mathrm{NMR}\left(\mathrm{CDCl}_{3}, 300 \mathrm{MHz}\right): \delta 8.13(\mathrm{~s}, 1 \mathrm{H}), 7.97-7.91(\mathrm{~m}, 3 \mathrm{H}), 7.64-$ $7.58(\mathrm{~m}, 3 \mathrm{H})$. Exact mass (El+) calcd for $\mathrm{C}_{14} \mathrm{H}_{7} \mathrm{~F}_{8} \mathrm{Br} 405.9603$, found 431.9727 .

\section{1-(4-bromo-1,1,2,2,3,3,4,4-octafluorobutyl)naphthalene}

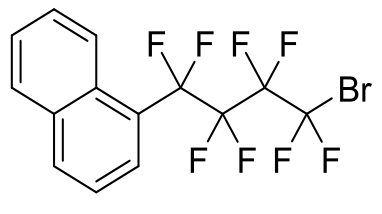

Synthesised following general procedure scheme 2 from 1-iodonaphthalene ( $0.10 \mathrm{mmol}$ scale) to yield $98 \%$ by internal standard, $\alpha, \alpha, \alpha$-trifluorotoluene as a white solid. Analytical data for 2-(4-bromo-1,1,2,2,3,3,4,4octafluorobutyl)naphthalene (7i) ${ }^{19} \mathrm{~F} \mathrm{NMR}\left(\mathrm{CDCl}_{3}, 470 \mathrm{MHz}\right): \delta \quad-63.90(\mathrm{t}, J=13.8 \mathrm{~Hz}, 2 \mathrm{~F}),-105.46(\mathrm{t}, J=13.8 \mathrm{~Hz}, 2 \mathrm{~F})$, $117.67(\mathrm{t}, J=16.0 \mathrm{~Hz}, 2 \mathrm{~F}),-120.40(\mathrm{t}, J=13.8 \mathrm{~Hz}, 2 \mathrm{~F}) .{ }^{1} \mathrm{H}$ NMR $\left(\mathrm{CDCl}_{3}, 300 \mathrm{MHz}\right): \delta 8.24(\mathrm{~d}, J=8.7 \mathrm{~Hz}, 1 \mathrm{H}), 8.06(\mathrm{~d}, J=8.2$ $\mathrm{Hz}, 1 \mathrm{H}), 7.92(\mathrm{~d}, J=8.3 \mathrm{~Hz}, 1 \mathrm{H}), 7.83(\mathrm{~d}, J=7.3 \mathrm{~Hz}, 1 \mathrm{H}), 7.61(\mathrm{t}, J=7.7 \mathrm{~Hz}, 1 \mathrm{H}), 7.56(\mathrm{t}, J=7.9 \mathrm{~Hz}, 2 \mathrm{H})$. Exact mass (El+) calcd for $\mathrm{C}_{14} \mathrm{H}_{7} \mathrm{~F}_{8} \mathrm{Br} 405.9603$, found 405.9581 . 
4-(4-bromo-1,1,2,2,3,3,4,4-octafluorobutyl)toluene<smiles>Cc1ccc(C(F)(F)C(F)(F)C(F)(F)C(F)(F)Br)cc1</smiles>

Synthesised following general procedure scheme 2 from 4 -iodotoluene $(0.10 \mathrm{mmol}$ scale) to yield $89 \%$ by internal standard, $\alpha, \alpha, \alpha$-trifluorotoluene as a colorless oil. Analytical data for 4-(4-bromo-1,1,2,2,3,3,4,4-octafluorobutyl)toluene (7j) ${ }^{19} \mathrm{~F} \mathrm{NMR}\left(\mathrm{MeCN}-d_{3}, 470 \mathrm{MHz}\right): \delta-65.69(\mathrm{t}, J=13.7 \mathrm{~Hz}, 2 \mathrm{~F}),-111.12(\mathrm{t}, J=13.8 \mathrm{~Hz}, 2 \mathrm{~F}),-117.82(\mathrm{~s}, 2 \mathrm{~F}),-122.16(\mathrm{~s}, 2 \mathrm{~F})$. ${ }^{1} \mathrm{H}$ NMR (MeCN- $\left.d_{3}, 300 \mathrm{MHz}\right): \delta 7.58(\mathrm{~d}, J=8.2 \mathrm{~Hz}, 2 \mathrm{H}), 6.97(\mathrm{~d}, J=7.9 \mathrm{~Hz}, 2 \mathrm{H}), 2.27(\mathrm{~s}, 3 \mathrm{H})$. Exact mass (El+) calcd for $\mathrm{C}_{11} \mathrm{H}_{7} \mathrm{~F}_{8} \mathrm{Br}$ 369.9603, found 369.9606 .

\section{2-(4-bromo-1,1,2,2,3,3,4,4-octafluorobutyl)toluene}<smiles>Cc1ccccc1C(F)(F)C(F)(F)C(F)(F)C(F)(F)Br</smiles>

Synthesised following general procedure scheme 2 from 2 -iodotoluene $(0.10 \mathrm{mmol}$ scale) to yield $83 \%$ by internal standard, $\alpha, \alpha, \alpha$-trifluorotoluene as a colorless oil. Analytical data for 2-(4-bromo-1,1,2,2,3,3,4,4-octafluorobutyl)toluene (7k) $\left.{ }^{19} \mathrm{~F} \mathrm{NMR} \mathrm{(MeCN-} d_{3}, 470 \mathrm{MHz}\right): \delta-65.57$ (t, $\left.J=13.9 \mathrm{~Hz}, 2 \mathrm{~F}\right),-106.87(\mathrm{t}, J=15.2 \mathrm{~Hz}, 2 \mathrm{~F}),-118.04(\mathrm{t}, J=16.6 \mathrm{~Hz}, 2 \mathrm{~F}),-$ $121.00(\mathrm{~s}, 2 \mathrm{~F}) .{ }^{1} \mathrm{H}$ NMR (MeCN-d $\left.3,300 \mathrm{MHz}\right): \delta 7.53(\mathrm{dd}, J=22.1,7.8 \mathrm{~Hz}, 2 \mathrm{H}), 7.38(\mathrm{~d}, J=3.9 \mathrm{~Hz}, 2 \mathrm{H}), 2.47$ (s, 3H). Exact mass (El+) calcd for $\mathrm{C}_{11} \mathrm{H}_{7} \mathrm{~F}_{8} \mathrm{Br} 369.9603$, found 369.9579 .

\section{1-(4-bromo-1,1,2,2,3,3,4,4-octafluorobutyl)-3,5-dimethylbenzene}

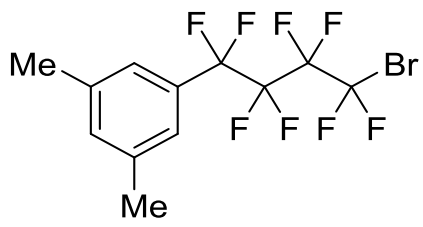

Synthesised following general procedure scheme 2 from 1-iodo-3,5-dimethylbenzene $(0.10 \mathrm{mmol}$ scale) to yield $91 \%$ by internal standard, $\alpha, \alpha, \alpha$-trifluorotoluene as a colorless oil. Analytical data for 1-(4-bromo-1,1,2,2,3,3,4,4-octafluorobutyl)3,5-dimethylbenzene (ㄱ) ${ }^{19} \mathrm{~F} \mathrm{NMR} \mathrm{(MeCN-} d_{3}, 470 \mathrm{MHz}$ ): $\delta-65.66$ (t, $\left.J=14.1 \mathrm{~Hz}, 2 \mathrm{~F}\right),-111.18$ (t, $\left.J=14.3 \mathrm{~Hz}, 2 \mathrm{~F}\right),-117.86$ (t, $J=14.1 \mathrm{~Hz}, 2 \mathrm{~F}),-121.99(\mathrm{t}, J=11.4 \mathrm{~Hz}, 2 \mathrm{~F}) .{ }^{1} \mathrm{H} \mathrm{NMR}\left(\mathrm{MeCN}-d_{3}, 300 \mathrm{MHz}\right): \delta 7.30(\mathrm{~s}, 1 \mathrm{H}), 7.26(\mathrm{~s}, 2 \mathrm{H}), 2.36(\mathrm{~s}, 6 \mathrm{H})$. Exact mass (El+) calcd for $\mathrm{C}_{12} \mathrm{H}_{9} \mathrm{~F}_{8} \mathrm{Br} 383.9760$, found 383.9786.

\section{1-(4-bromo-1,1,2,2,3,3,4,4-octafluorobutyl)-4-tertbutylbenzene}

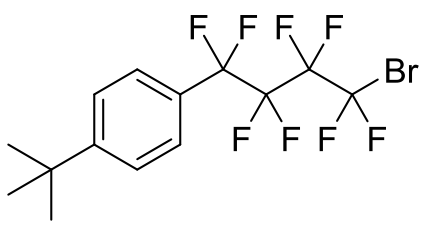

Synthesised following general procedure scheme 2 from 1-iodo-4-tertbutylbenzene $(0.10 \mathrm{mmol}$ scale) to yield $82 \%$ by internal standard, $\alpha, \alpha, \alpha$-trifluorotoluene as a colorless oil. Analytical data for 1-(4-bromo-1,1,2,2,3,3,4,4-octafluorobutyl)4-tertbutylbenzene $(7 \mathrm{~m}){ }^{19} \mathrm{~F} N M R\left(\mathrm{MeCN}-d_{3}, 470 \mathrm{MHz}\right): \delta$-65.68 (s, 2F), -111.12 (s, 2F), -117.82 (s, 2F), -122.08 (s, 2F). ${ }^{1} \mathrm{H}$ $\operatorname{NMR}\left(\mathrm{MeCN}-d_{3}, 300 \mathrm{MHz}\right): \delta 7.63(\mathrm{~d}, J=8.6 \mathrm{~Hz}, 2 \mathrm{H}), 7.21(\mathrm{~d}, J=8.6 \mathrm{~Hz}, 2 \mathrm{H}), 1.27(\mathrm{~s}, 9 \mathrm{H})$. Exact mass (El+) calcd for $\mathrm{C}_{14} \mathrm{H}_{13} \mathrm{~F}_{8} \mathrm{Br}$ 412.0073, found 412.0063 . 
1-(4-bromo-1,1,2,2,3,3,4,4-octafluorobutyl)anisole

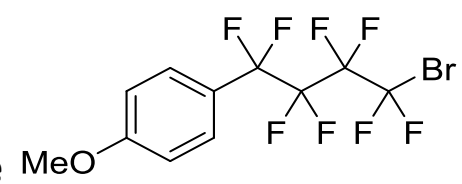

Synthesised following general procedure scheme 2 from 4-iodoanisole $(0.50 \mathrm{mmol}$ scale). Purification by flash silica column chromatography (eluent: $50 \%$ dichloromethane in hexanes) to yield $83 \%$ by internal standard, $\alpha, \alpha, \alpha-$ trifluorotoluene $(54.2 \mathrm{mg}, 28 \%$ yield isolated yield) as a colorless oil. Analytical data for 1-(4-bromo-1,1,2,2,3,3,4,4octafluorobutyl)anisole $(7 \mathrm{n}){ }^{19} \mathrm{~F} \mathrm{NMR}\left(\mathrm{CDCl}_{3}, 470 \mathrm{MHz}\right): \delta-64.01(\mathrm{t}, J=13.8 \mathrm{~Hz}, 2 \mathrm{~F}),-110.74(\mathrm{t}, J=13.8 \mathrm{~Hz}, 2 \mathrm{~F}),-117.61(\mathrm{t}$, $J=13.7 \mathrm{~Hz}, 2 \mathrm{~F}),-122.16(\mathrm{t}, J=13.7 \mathrm{~Hz}, 2 \mathrm{~F}) .{ }^{1} \mathrm{H}$ NMR $\left(\mathrm{MeCN}-d_{3}, 300 \mathrm{MHz}\right): \delta 7.51(\mathrm{~d}, J=8.8 \mathrm{~Hz}, 2 \mathrm{H}), 6.99(\mathrm{~d}, J=8.9 \mathrm{~Hz}, 2 \mathrm{H})$, $3.86(\mathrm{~s}, 3 \mathrm{H})$. Exact mass (El+) calcd for $\mathrm{C}_{11} \mathrm{H}_{7} \mathrm{OF}_{8} \mathrm{Br} 385.9553$, found 385.9557.

\section{4-(4-bromo-1,1,2,2,3,3,4,4-octafluorobutyl)nitrobenzene $\mathrm{O}_{2} \mathrm{~N}$}

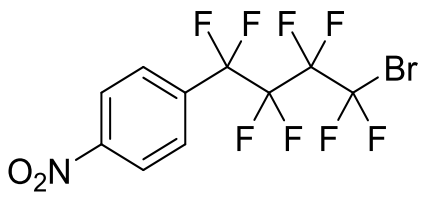

Synthesised following general procedure scheme 2 from 4 -iodonitrobenzene $(0.50 \mathrm{mmol}$ scale). Purification by flash silica column chromatography (eluent: $50 \%$ dichloromethane in hexanes) to yield $>99 \%$ by internal standard, $\alpha, \alpha, \alpha-$ trifluorotoluene $(176.9 \mathrm{mg}, 88 \%$ yield isolated yield) as a white solid. Analytical data for 4-(4-bromo-1,1,2,2,3,3,4,4octafluorobutyl)nitrobenzene (7o) ${ }^{19} \mathrm{~F} \mathrm{NMR}\left(\mathrm{CDCl}_{3}, 470 \mathrm{MHz}\right): \delta \quad-64.35(\mathrm{t}, J=13.9 \mathrm{~Hz}, 2 \mathrm{~F}),-112.17(\mathrm{t}, \mathrm{J}=14.3 \mathrm{~Hz}, 2 \mathrm{~F}),-$ $117.50(\mathrm{t}, J=16.0 \mathrm{~Hz}, 2 \mathrm{~F}),-121.83(\mathrm{t}, J=14.1 \mathrm{~Hz}, 2 \mathrm{~F}) .{ }^{1} \mathrm{H} \mathrm{NMR}\left(\mathrm{CDCl}_{3}, 300 \mathrm{MHz}\right): \delta 8.38(\mathrm{~d}, J 8.5 \mathrm{~Hz}, 2 \mathrm{H}), 7.82(\mathrm{~d}, J=8.6 \mathrm{~Hz}$, $2 \mathrm{H})$. Exact mass (El+) calcd for $\mathrm{C}_{10} \mathrm{H}_{4} \mathrm{NO}_{2} \mathrm{~F}_{8} \mathrm{Br} 402.9328$, found 402.9317.

\section{4-(4-bromo-1,1,2,2,3,3,4,4-octafluorobutyl)benzonitrile}

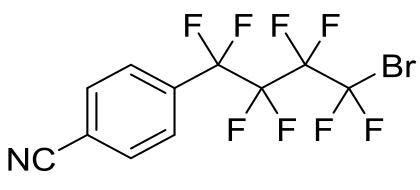

Synthesised following general procedure scheme 2 from 4-iodobenzonitrile $(0.50 \mathrm{mmol}$ scale). Purification by flash silica column chromatography (eluent: $50 \%$ dichloromethane in hexanes) to yield $>99 \%$ by internal standard, $\alpha, \alpha, \alpha-$ trifluorotoluene $(152.8 \mathrm{mg}, 80 \%$ yield isolated yield) as a white solid. Analytical data for 4-(4-bromo-1,1,2,2,3,3,4,4octafluorobutyl)benzonitrile (7p) ${ }^{19} \mathrm{~F} \mathrm{NMR}\left(\mathrm{CDCl}_{3}, 470 \mathrm{MHz}\right):-64.33(\mathrm{t}, J=14.3 \mathrm{~Hz}, 2 \mathrm{~F}),-112.64(\mathrm{t}, J=14.3 \mathrm{~Hz}, 2 \mathrm{~F}),-117.54$ (t, $J=15.5 \mathrm{~Hz}, 2 \mathrm{~F}),-121.92(\mathrm{t}, J=14.0 \mathrm{~Hz}, 2 \mathrm{~F}) .{ }^{1} \mathrm{H}$ NMR $\left(\mathrm{CDCl}_{3}, 300 \mathrm{MHz}\right): \delta 7.83(\mathrm{~s}, J=8.1 \mathrm{~Hz}, 2 \mathrm{H}), 7.73(\mathrm{~d}, J=8.4 \mathrm{~Hz}, 2 \mathrm{H})$. Exact mass (El+) calcd for $\mathrm{C}_{11} \mathrm{H}_{4} \mathrm{NF}_{8} \mathrm{Br} 380.9399$, found 380.9427 .

\section{methyl 4-(4-bromo-1,1,2,2,3,3,4,4-octafluorobutyl)benzoate}

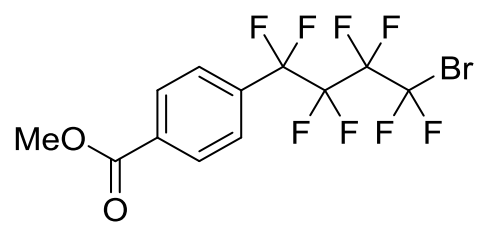

Synthesised following general procedure scheme 2 from methyl 4-iodobenzoate $(0.50 \mathrm{mmol}$ scale). Purification by flash silica column chromatography (eluent: $50 \%$ dichloromethane in hexanes) to yield $96 \%$ by internal standard, $\alpha, \alpha, \alpha-$ trifluorotoluene (157.7 mg, 76\% yield isolated yield) as a white solid. Analytical data for methyl 4-(4-bromo-1,1,2,2,3,3,4,4octafluorobutyl)benzoate (7q) $\left.{ }^{19} \mathrm{~F} \mathrm{NMR} \mathrm{(CDCl} 3,470 \mathrm{MHz}\right): \delta-64.18(\mathrm{t}, J=14.4 \mathrm{~Hz}, 2 \mathrm{~F}),-112.22(\mathrm{t}, J=14.3 \mathrm{~Hz}, 2 \mathrm{~F}),-117.56$ $(\mathrm{t}, J=15.1 \mathrm{~Hz}, 2 \mathrm{~F}),-122.02(\mathrm{t}, J=13.2 \mathrm{~Hz}, 2 \mathrm{~F}) .{ }^{1} \mathrm{H}$ NMR $\left(\mathrm{CDCl}_{3}, 300 \mathrm{MHz}\right): \delta 8.17(\mathrm{~d}, J=8.7 \mathrm{~Hz}, 2 \mathrm{H}), 7.68(\mathrm{~d}, J=8.3 \mathrm{~Hz}, 2 \mathrm{H})$, $3.96(\mathrm{~s}, 3 \mathrm{H})$. Exact mass (El+) calcd for $\mathrm{C}_{12} \mathrm{H}_{7} \mathrm{O}_{2} \mathrm{~F}_{8} \mathrm{Br} 413.9502$, found 413.9530 . 
ethyl 4-(4-bromo-1,1,2,2,3,3,4,4-octafluorobutyl)benzoate

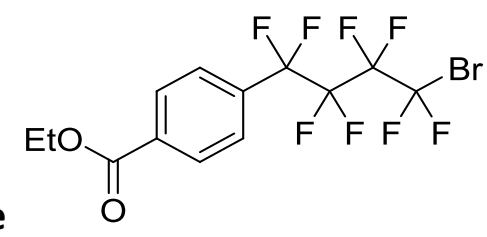

Synthesised following general procedure scheme 2 from ethyl 4 -iodobenzoate $(0.50 \mathrm{mmol}$ scale $)$. Purification by flash silica column chromatography (eluent: $50 \%$ dichloromethane in hexanes) to yield $>99 \%$ by internal standard, $\alpha, \alpha, \alpha-$ trifluorotoluene ( $148.0 \mathrm{mg}, 69 \%$ yield isolated yield) as a white solid. Analytical data for ethyl 4-(4-bromo-1,1,2,2,3,3,4,4octafluorobutyl)benzoate (7r) ${ }^{19} \mathrm{~F} \mathrm{NMR}\left(\mathrm{CDCl}_{3}, 470 \mathrm{MHz}\right):-64.18(\mathrm{t}, J=14.2 \mathrm{~Hz}, 2 \mathrm{~F}),-112.20(\mathrm{t}, J=14.1 \mathrm{~Hz}, 2 \mathrm{~F}),-117.55(\mathrm{t}$, $J=14.0 \mathrm{~Hz}, 2 \mathrm{~F}),-122.04(\mathrm{t}, J=13.7 \mathrm{~Hz}, 2 \mathrm{~F}) .{ }^{1} \mathrm{H} \mathrm{NMR}\left(\mathrm{CDCl}_{3}, 300 \mathrm{MHz}\right): \delta 8.17(\mathrm{~d}, J=8.1 \mathrm{~Hz}, 2 \mathrm{H}), 7.68(\mathrm{~d}, J=8.2 \mathrm{~Hz}, 2 \mathrm{H})$, $4.42(q, J=7.1 \mathrm{~Hz}, 2 \mathrm{H}), 1.42(\mathrm{~d}, J=7.1 \mathrm{~Hz}, 3 \mathrm{H})$. Exact mass $(\mathrm{El}+)$ calcd for $\mathrm{C}_{13} \mathrm{H}_{9} \mathrm{O}_{2} \mathrm{~F}_{8} \mathrm{Br}$ 427.9658, found 427.9655 .

\section{1-(4-bromo-1,1,2,2,3,3,4,4-octafluorobutyl)-4-(4-morpholinyl)benzene}<smiles>FC(F)(Br)C(F)(F)C(F)(F)C(F)(F)c1ccc(N2CCOCC2)cc1</smiles>

Synthesised following general procedure scheme 2 from 4-(4-iodophenyl)morpholine $(0.50 \mathrm{mmol}$ scale). Purification by flash silica column chromatography (eluent: dichloromethane) to yield $97 \%$ by internal standard, $\alpha, \alpha, \alpha$-trifluorotoluene (145.9 mg, 66\% yield isolated yield) as a white solid. Analytical data for 1-(4-bromo-1,1,2,2,3,3,4,4-octafluorobutyl)-4-(4morpholinyl)benzene (7s) ${ }^{19} \mathrm{~F} \mathrm{NMR}\left(\mathrm{CDCl}_{3}, 470 \mathrm{MHz}\right): \delta-63.96(\mathrm{t}, J=14.0 \mathrm{~Hz}, 2 \mathrm{~F}),-110.78(\mathrm{t}, J=13.9 \mathrm{~Hz}, 2 \mathrm{~F}),-117.61(\mathrm{t}, J$ $=13.8 \mathrm{~Hz}, 2 \mathrm{~F}),-122.18(\mathrm{t}, J=11.7 \mathrm{~Hz}, 2 \mathrm{~F}) .{ }^{1} \mathrm{H} \mathrm{NMR}\left(\mathrm{CDCl}_{3}, 300 \mathrm{MHz}\right): \delta 7.45(\mathrm{~d}, J=8.8 \mathrm{~Hz}, 2 \mathrm{H}), 6.94(\mathrm{~d}, J=9.0 \mathrm{~Hz}, 2 \mathrm{H}), 3.87$ $(\mathrm{t}, J=3.0 \mathrm{~Hz}, 4 \mathrm{H}), 3.26(\mathrm{t}, J=3.1 \mathrm{~Hz}, 4 \mathrm{H})$. Exact mass $(\mathrm{El}+)$ calcd for $\mathrm{C}_{14} \mathrm{H}_{12} \mathrm{NOF}_{8} \mathrm{Br} 440.9975$, found 440.9985 .

\section{4-(4-bromo-1,1,2,2,3,3,4,4-octafluorobutyl)-1-fluorobenzene}

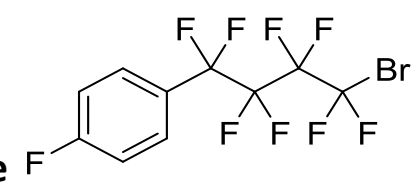

Synthesised following general procedure scheme 2 from 1-fluoro-4-iodobenzene $(0.10 \mathrm{mmol}$ scale) to yield $78 \%$ by internal standard, $\alpha, \alpha, \alpha$-trifluorotoluene as a colorless oil. Analytical data for 4-(4-bromo-1,1,2,2,3,3,4,4-octafluorobutyl)1-fluorobenzene (7t) ${ }^{19} \mathrm{~F} \mathrm{NMR} \mathrm{(MeCN-} d_{3}, 470 \mathrm{MHz}$ ): $\delta$-65.76 (s, 2F), -109.28 (s, 1F), -110.78 (s, 2F), -117.83 (s, 2F), -112.10 (s, 2F). ${ }^{1} \mathrm{H} \mathrm{NMR}\left(\mathrm{MeCN}-d_{3}, 300 \mathrm{MHz}\right): \delta 87.70(\mathrm{t}, J=8.6 \mathrm{~Hz}, 2 \mathrm{H}), 7.32(\mathrm{t}, J=8.8 \mathrm{~Hz}, 2 \mathrm{H})$. Exact mass $(\mathrm{El}+)$ calcd for $\mathrm{C}_{10} \mathrm{H}_{4} \mathrm{~F}_{9} \mathrm{Br}$ 373.9353, found 373.9349 .

\section{4-(4-bromo-1,1,2,2,3,3,4,4-octafluorobutyl)-1-chlorobenzene}

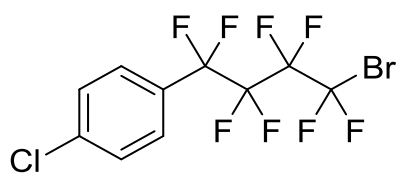

Synthesised following general procedure scheme 2 from 1-chloro-4-iodobenzene $(0.10 \mathrm{mmol}$ scale) to yield $80 \%$ by internal standard, $\alpha, \alpha, \alpha$-trifluorotoluene as a colorless oil. Analytical data for 4-(4-bromo-1,1,2,2,3,3,4,4-octafluorobutyl)1-chlorobenzene $(7 \mathrm{u}){ }^{19} \mathrm{~F} \mathrm{NMR}\left(\mathrm{MeCN}-d_{3}, 470 \mathrm{MHz}\right): \delta-65.80(\mathrm{t}, J=12.9 \mathrm{~Hz}, 2 \mathrm{~F}),-111.49(\mathrm{t}, J=13.3 \mathrm{~Hz}, 2 \mathrm{~F}),-117.82(\mathrm{~s}, 2 \mathrm{~F})$, $-122.13(\mathrm{~s}, 2 \mathrm{~F}) .{ }^{1} \mathrm{H}$ NMR (MeCN-d $\left.d_{3}, 300 \mathrm{MHz}\right): \delta 7.62(\mathrm{q}, J=8.6 \mathrm{~Hz}, 4 \mathrm{H})$. Exact mass (El+) calcd for $\mathrm{C}_{10} \mathrm{H}_{4} \mathrm{~F}_{8} \mathrm{ClBr} 389.9057$, found 389.9076 . 


\section{4-(4-bromo-1,1,2,2,3,3,4,4-octafluorobutyl)-1-bromobenzene $\mathrm{Br}$}

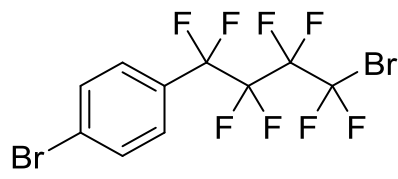

Synthesised following general procedure scheme 2 from 1-bromo-4-iodobenzene $(0.10 \mathrm{mmol}$ scale $)$ to yield $97 \%$ by internal standard, $\alpha, \alpha, \alpha$-trifluorotoluene as a colorless oil. Analytical data for 4-(4-bromo-1,1,2,2,3,3,4,4-octafluorobutyl)1-bromobenzene (7v) ${ }^{19} \mathrm{~F} \mathrm{NMR}\left(\mathrm{MeCN}-d_{3}, 470 \mathrm{MHz}\right): \delta-65.81(\mathrm{t}, J=12.5 \mathrm{~Hz}, 2 \mathrm{~F}),-111.67(\mathrm{t}, J=12.9 \mathrm{~Hz}, 2 \mathrm{~F}),-117.82(\mathrm{~s}, 2 \mathrm{~F})$, $-122.14(\mathrm{~s}, 2 \mathrm{~F}) .{ }^{1} \mathrm{H}$ NMR (MeCN-d $d_{3}, 300 \mathrm{MHz}$ ): $\delta 7.76(\mathrm{~d}, J=8.5 \mathrm{~Hz}, 2 \mathrm{H}), 7.56(\mathrm{~d}, J=8.4 \mathrm{~Hz}, 2 \mathrm{H}$ ). Exact mass (El+) calcd for $\mathrm{C}_{10} \mathrm{H}_{4} \mathrm{~F}_{8} \mathrm{Br}_{2}$ 433.8552, found 433.8578 .

\section{4-(4-bromo-1,1,2,2,3,3,4,4,5,5,6,6-dodecafluorohexyl)nitrobenzene}<smiles>O=[N+]([O-])c1ccc(C(F)(F)C(F)(F)C(F)(F)C(F)(F)C(F)(F)C(F)(F)Br)cc1</smiles>

Synthesised following general procedure scheme 2 from 4-iodonitrobenzene $(0.10 \mathrm{mmol}$ scale). Purification by flash silica column chromatography (eluent: $50 \%$ dichloromethane in hexanes) to yield $82 \%$ by internal standard $\alpha, \alpha, \alpha-$ trifluorotoluene $(30.1 \mathrm{mg}, 60 \%$ yield isolated yield) as a white solid. Analytical data for 4-(4-bromo-1,1,2,2,3,3,4,4,5,5,6,6dodecafluorohexyl)nitrobenzene (10a) ${ }^{19} \mathrm{~F} \mathrm{NMR}\left(\mathrm{CDCl}_{3}, 470 \mathrm{MHz}\right): \delta \quad-64.45(\mathrm{t}, J=13.2 \mathrm{~Hz}, 2 \mathrm{~F}),-112.12(\mathrm{t}, J=13.1 \mathrm{~Hz}$, 2F), -118.25 (s, 2F), -122.09 (s, 4F), $-122.54(\mathrm{~s}, 2 \mathrm{~F}) .{ }^{1} \mathrm{H}$ NMR $\left(\mathrm{CDCl}_{3}, 300 \mathrm{MHz}\right): \delta 8.38(\mathrm{~d}, J=8.5 \mathrm{~Hz}, 2 \mathrm{H}), 7.82(\mathrm{~d}, J=8.6 \mathrm{~Hz}$, $2 \mathrm{H})$. Exact mass (El+) calcd for $\mathrm{C}_{12} \mathrm{H}_{4} \mathrm{NO}_{2} \mathrm{~F}_{12} \mathrm{Br} 500.9234$, found 500.9220 .

\section{4-(4-bromo-1,1,2,2,3,3,4,4,5,5,6,6-dodecafluorohexyl)benzonitrile}<smiles>N#Cc1ccc(C(F)(F)C(F)(F)C(F)(F)C(F)(F)C(F)(F)C(F)(F)Br)cc1</smiles>

Synthesised following general procedure scheme 2 from 4-iodobenzonitrile $(0.10 \mathrm{mmol}$ scale). Purification by flash silica column chromatography (eluent: $50 \%$ dichloromethane in hexanes) to yield $84 \%$ by internal standard, $\alpha, \alpha, \alpha-$ trifluorotoluene ( $26.5 \mathrm{mg}, 55 \%$ yield isolated yield) as a white solid. Analytical data for 4-(4-bromo-1,1,2,2,3,3,4,4,5,5,6,6dodecafluorohexyl)benzonitirle (10b) ${ }^{19} \mathrm{~F} \mathrm{NMR}\left(\mathrm{CDCl}_{3}, 470 \mathrm{MHz}\right): \delta-64.45(\mathrm{t}, J=13.3 \mathrm{~Hz}, 2 \mathrm{~F}),-112.58(\mathrm{t}, J=13.1 \mathrm{~Hz}, 2 \mathrm{~F})$, $118.24(\mathrm{~s}, 2 \mathrm{~F}),-122.11(\mathrm{~s}, 4 \mathrm{~F}),-122.61(\mathrm{~s}, 2 \mathrm{~F}) .{ }^{1} \mathrm{H}$ NMR $\left(\mathrm{CDCl}_{3}, 300 \mathrm{MHz}\right): 7.83(\mathrm{~d}, J=8.3 \mathrm{~Hz}, 2 \mathrm{H}), 7.74(\mathrm{~d}, J=8.3 \mathrm{~Hz}, 2 \mathrm{H})$. Exact mass (El+) calcd for $\mathrm{C}_{13} \mathrm{H}_{4} \mathrm{NF}_{12} \mathrm{Br} 480.9335$, found 480.9328 . 
methyl 4-(4-bromo-1,1,2,2,3,3,4,4,5,5,6,6-dodecafluorohexyl)benzoate<smiles>COC(=O)c1ccc(C(F)(F)C(F)(F)C(F)(F)C(F)(F)C(F)(F)C(F)(F)Br)cc1</smiles>

Synthesised following general procedure scheme 2 from methyl 4-iodobenzoate $(0.10 \mathrm{mmol}$ scale). Purification by flash silica column chromatography (eluent: $50 \%$ dichloromethane in hexanes) to yield $75 \%$ by internal standard, $\alpha, \alpha, \alpha-$ trifluorotoluene $(23.7 \mathrm{mg}, 46 \%$ yield isolated yield) as a white solid. Analytical data for methyl 4-(4-bromo1,1,2,2,3,3,4,4,5,5,6,6-dodecafluorohexyl)benzoate (10c) ${ }^{19} \mathrm{~F} \mathrm{NMR} \mathrm{(} \mathrm{CDCl}_{3}, 470 \mathrm{MHz}$ ): $\delta$-64.39 (t, J = 13.7 Hz, $\left.2 \mathrm{~F}\right),-112.16$ $(\mathrm{t}, J=13.4 \mathrm{~Hz}, 2 \mathrm{~F}),-118.25(\mathrm{~s}, 2 \mathrm{~F}),-122.15(\mathrm{~s}, 4 \mathrm{~F}),-122.75(\mathrm{~s}, 2 \mathrm{~F}) .{ }^{1} \mathrm{H}$ NMR $\left(\mathrm{CDCl}_{3}, 300 \mathrm{MHz}\right): \delta 8.17(\mathrm{~d}, J=8.6 \mathrm{~Hz}, 2 \mathrm{H}), 7.68$ $(\mathrm{d}, J=8.4 \mathrm{~Hz}, 2 \mathrm{H}), 3.96(\mathrm{~s}, 3 \mathrm{H})$. Exact mass (El+) calcd for $\mathrm{C}_{14} \mathrm{H}_{7} \mathrm{O}_{2} \mathrm{~F}_{12} \mathrm{Br} 513.9438$, found 513.9443 .

\section{4-(4-bromo-1,1,2,2,3,3,4,4,5,5,6,6-dodecafluorohexyl)biphenyl}<smiles>FC(F)(Br)C(F)(F)C(F)(F)C(F)(F)C(F)(F)C(F)(F)c1ccc(-c2ccccc2)cc1</smiles>

Synthesised following general procedure scheme 2 from 4-iodobiphenyl $(0.10 \mathrm{mmol}$ scale). Purification by flash silica column chromatography (eluent: hexanes) to yield $93 \%$ by internal standard, $\alpha, \alpha, \alpha$-trifluorotoluene ( $29.9 \mathrm{mg}, 56 \%$ yield isolated yield) as a white solid. Analytical data for 4-(4-bromo-1,1,2,2,3,3,4,4,5,5,6,6-dodecafluorohexyl)biphenyl (10d) ${ }^{19} \mathrm{~F}$ $\mathrm{NMR}\left(\mathrm{CDCl}_{3}, 470 \mathrm{MHz}\right): \delta-64.32(\mathrm{t}, J=13.7 \mathrm{~Hz}, 2 \mathrm{~F}),-111.53(\mathrm{t}, J=13.3 \mathrm{~Hz}, 2 \mathrm{~F}),-118.23(\mathrm{~s}, 2 \mathrm{~F}),-122.08(\mathrm{~s}, 2 \mathrm{~F}),-122.22(\mathrm{~s}$, 2F), $-122.73(\mathrm{~s}, 2 \mathrm{~F}) .{ }^{1} \mathrm{H} \mathrm{NMR}\left(\mathrm{CDCl}_{3}, 300 \mathrm{MHz}\right): \delta 7.72(\mathrm{~d}, J=8.4 \mathrm{~Hz}, 2 \mathrm{H}), 7.66(\mathrm{~d}, J=8.4 \mathrm{~Hz}, 2 \mathrm{H}), 7.62(\mathrm{~d}, J=8.4 \mathrm{~Hz}, 2 \mathrm{H}), 7.48$ $(\mathrm{t}, J=8.0 \mathrm{~Hz}, 2 \mathrm{H}), 7.42(\mathrm{tt}, J=8.4,1.0 \mathrm{~Hz}, 1 \mathrm{H})$. Exact mass (El+) calcd for $\mathrm{C}_{18} \mathrm{H}_{9} \mathrm{~F}_{12} \mathrm{Br}$ 531.9696, found 531.9697. 
2) Spectral data for all new compounds:

Figure S1: ${ }^{19} \mathrm{~F} N M R$ spectrum of

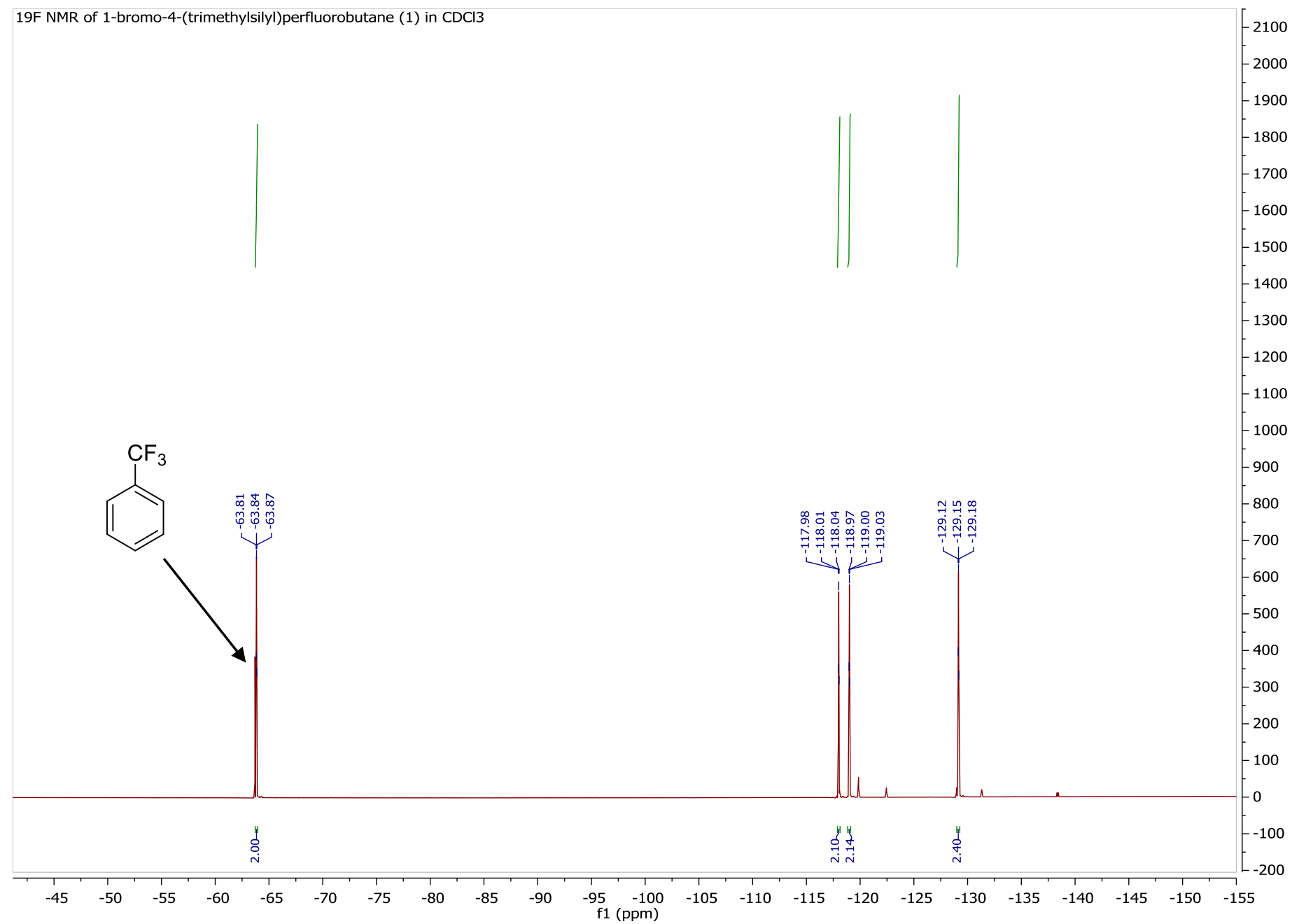



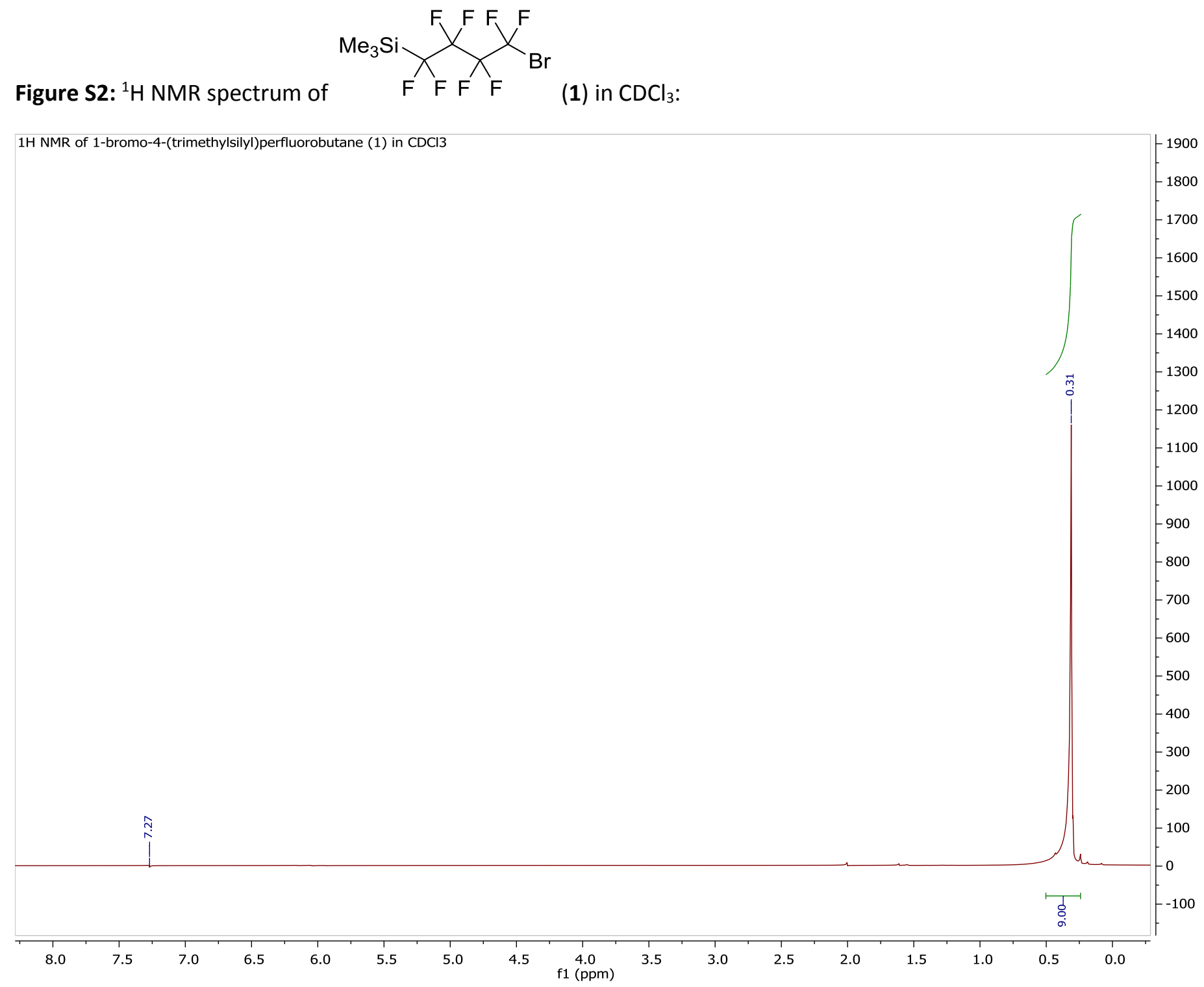
Figure S3: ${ }^{13} \mathrm{C}$ NMR spectrum of

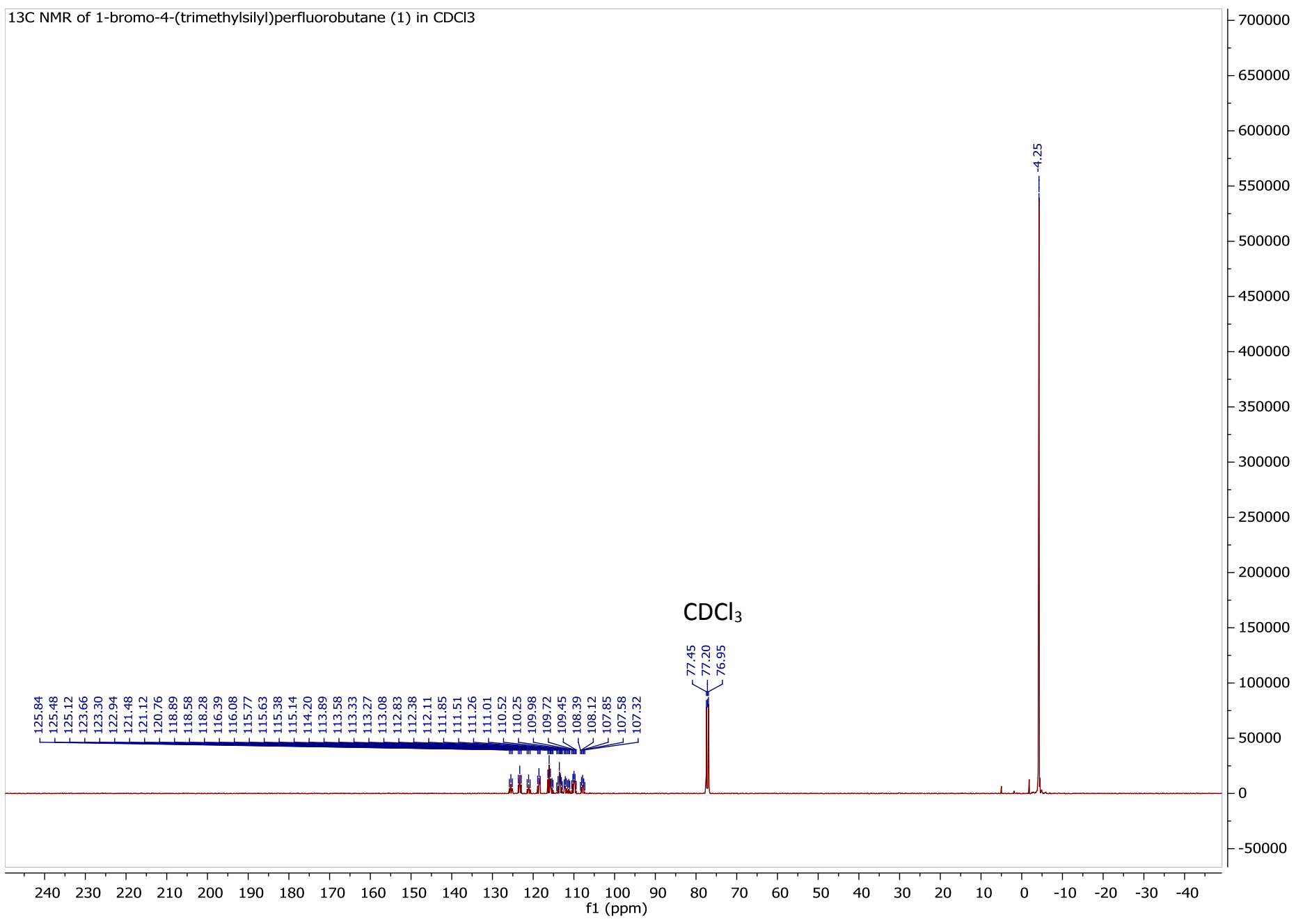


Figure S4: ${ }^{19} \mathrm{~F}$ NMR spectrum of

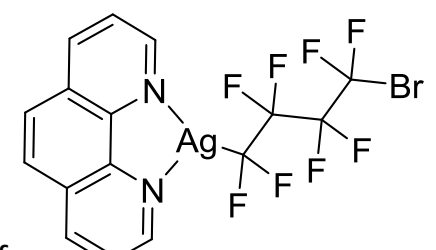

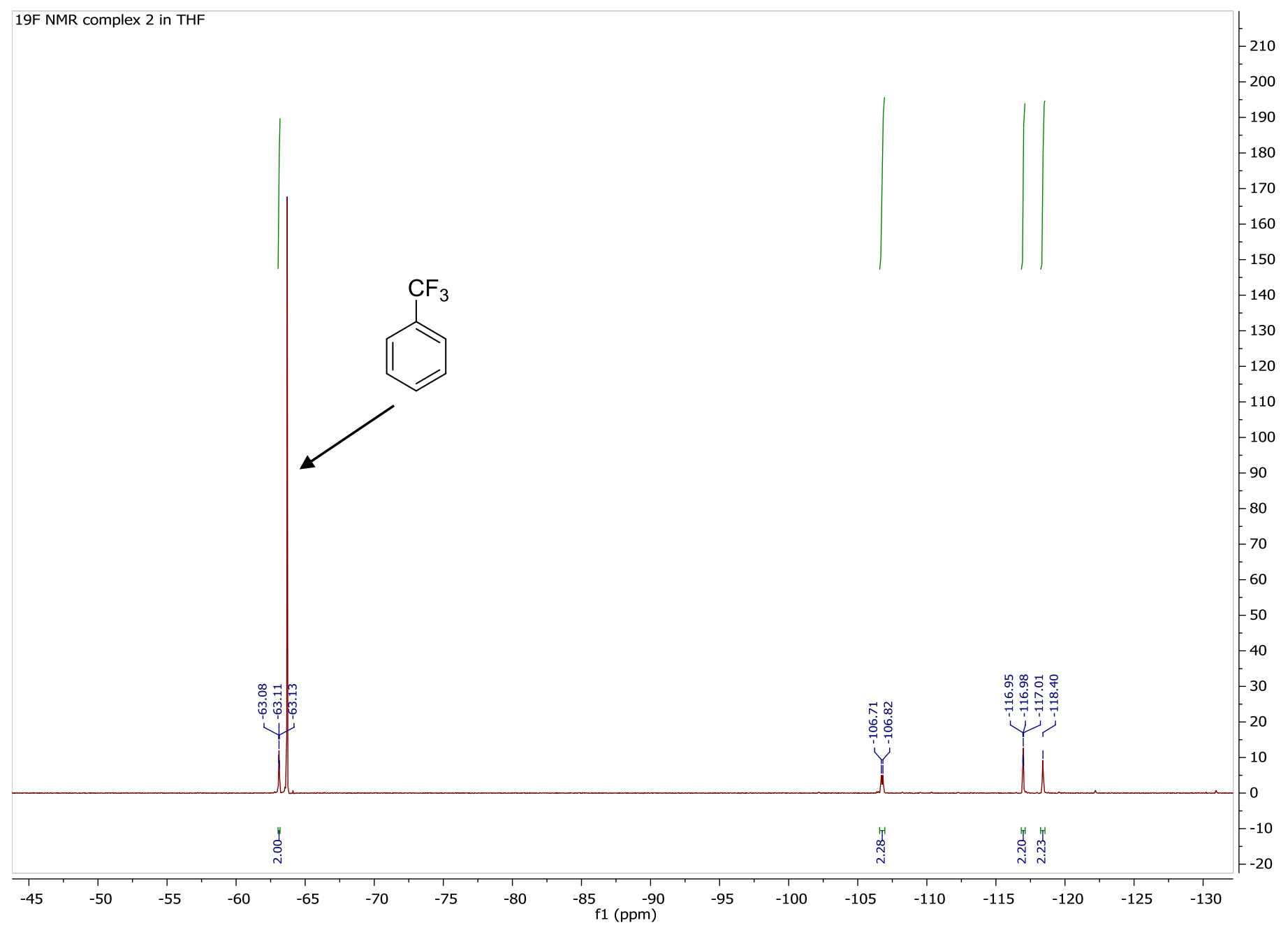


Figure S5: ${ }^{1} \mathrm{H}$ NMR spectrum of

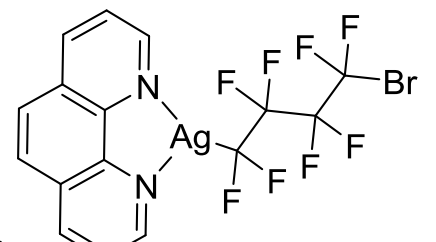

(2) in THF-d $\mathrm{d}_{8}$ :

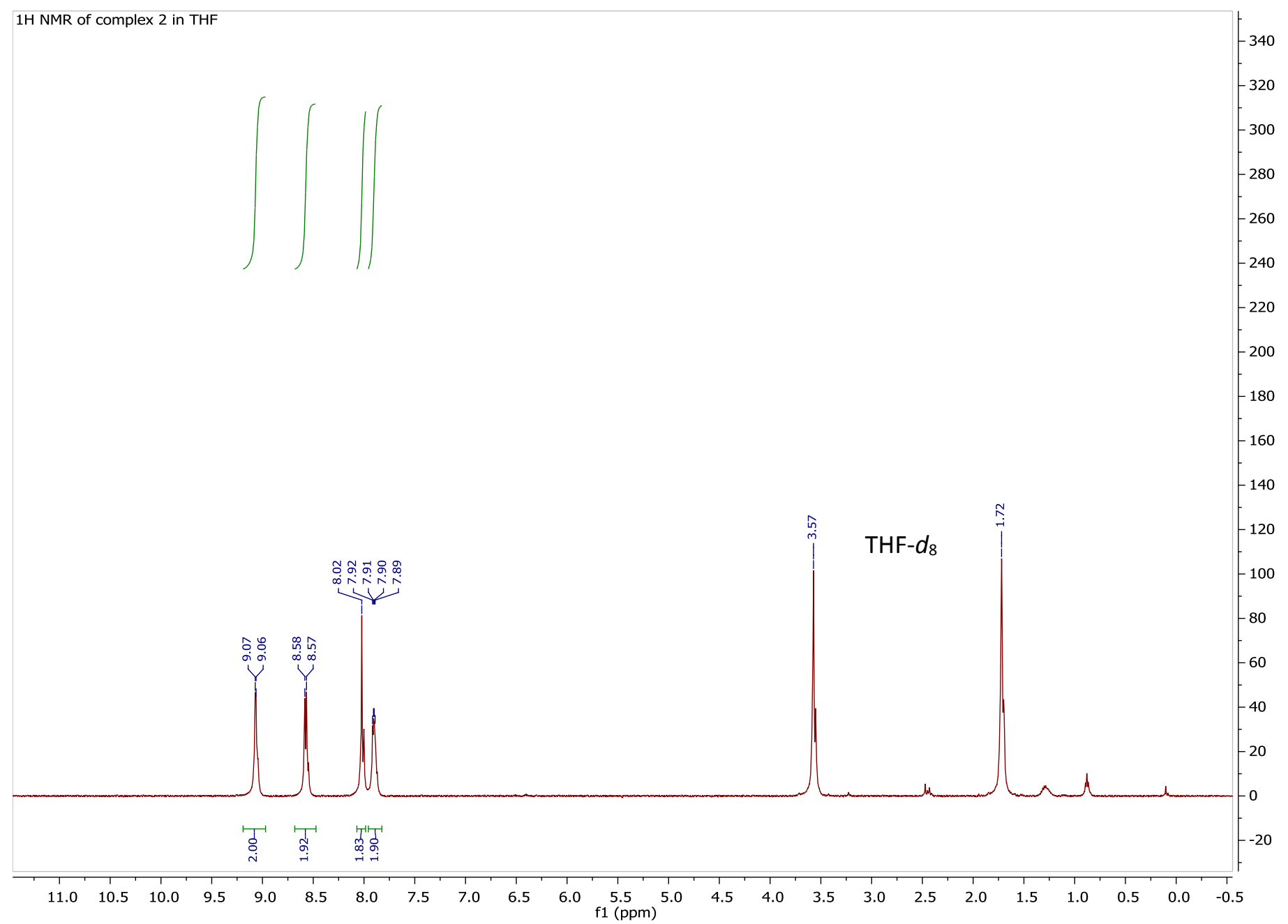


Figure S6: ${ }^{19} \mathrm{~F}$ NMR spectrum of

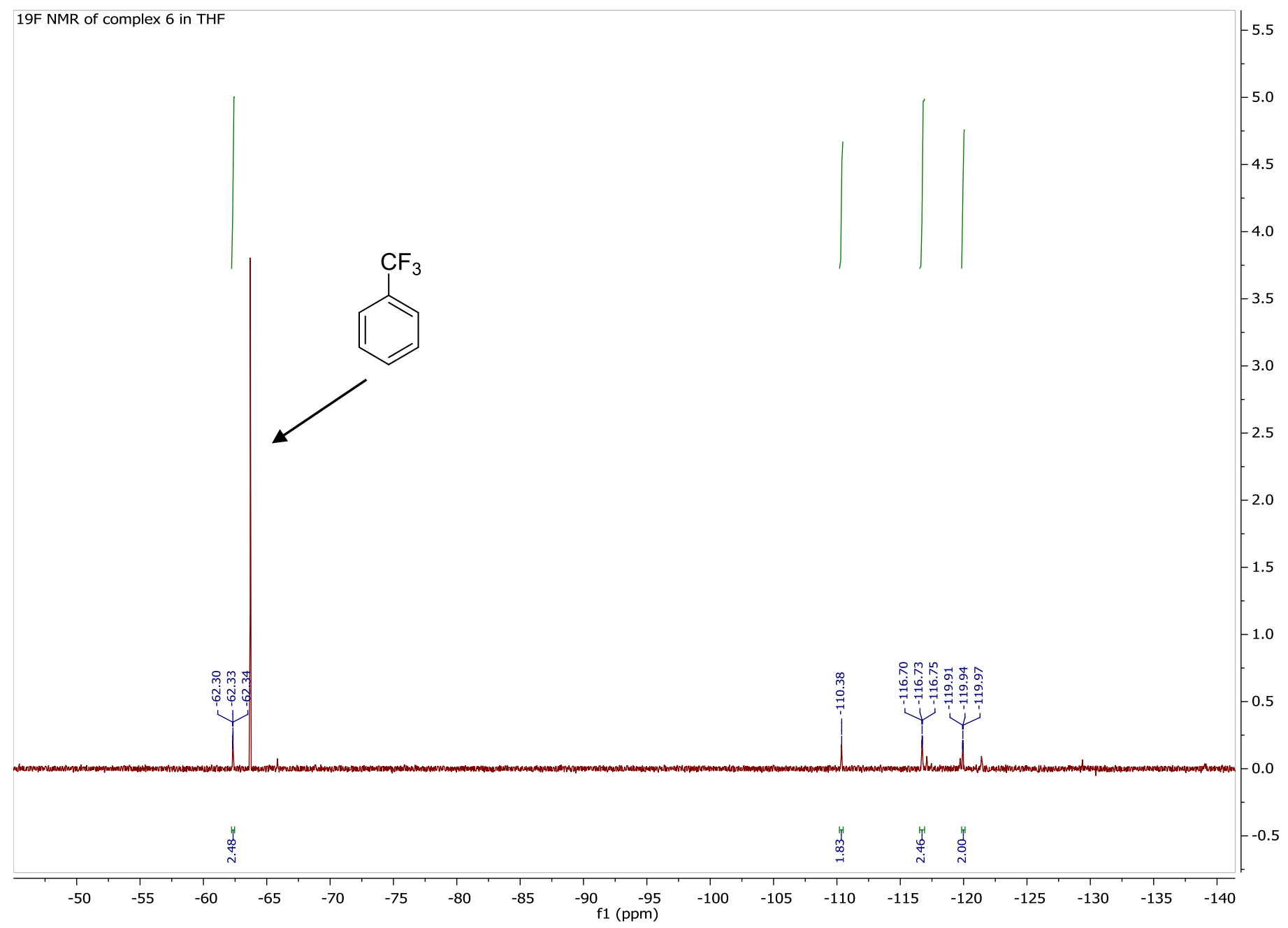

(6) in: THF- $d_{8}$<smiles></smiles> 
Figure S7: ${ }^{1} \mathrm{H}$ NMR spectrum of

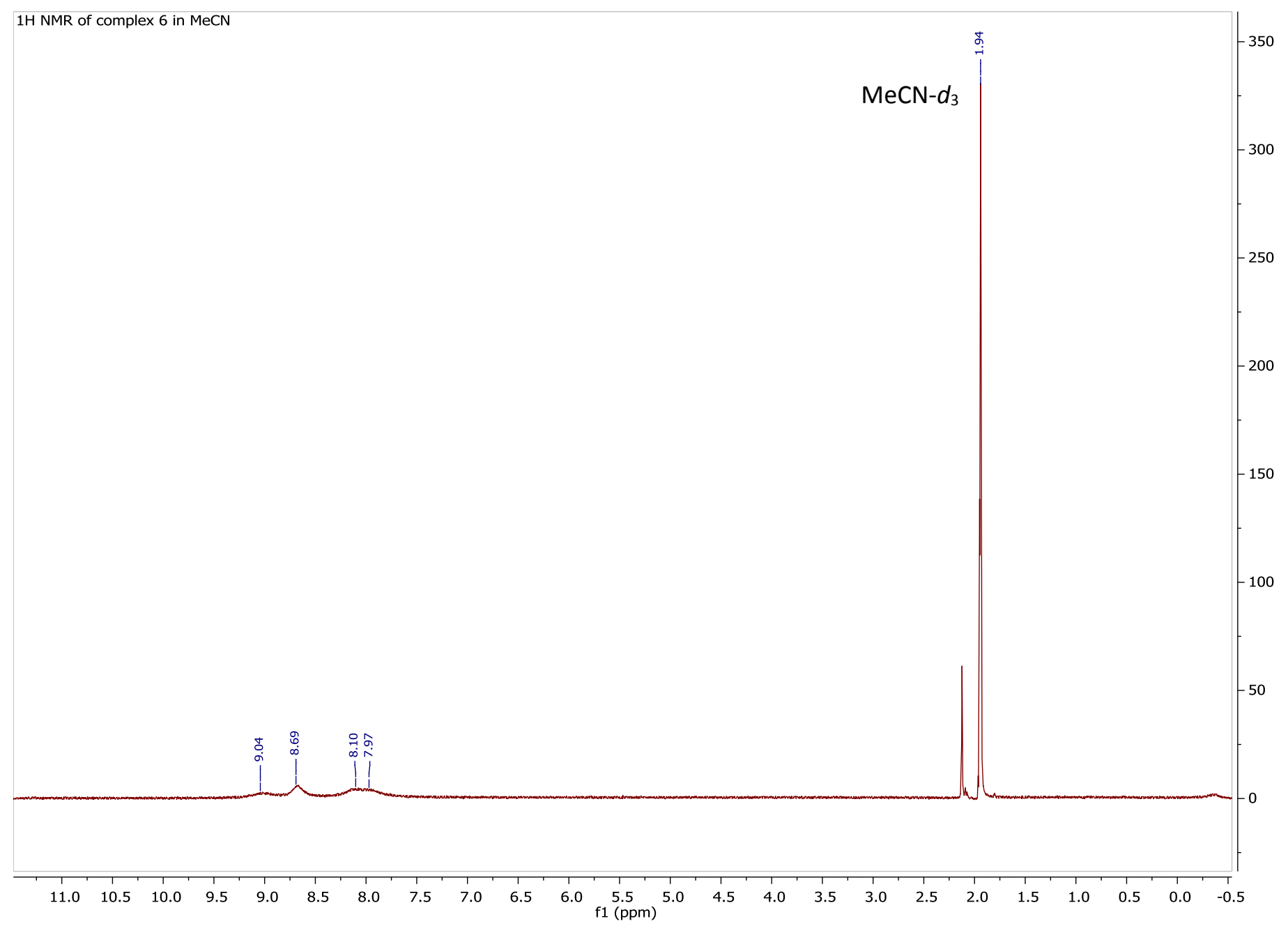

(6) in: $\mathrm{MeCN}-d_{3}$ 
Figure S8: ${ }^{19} \mathrm{~F} \mathrm{NMR}$ spectrum of

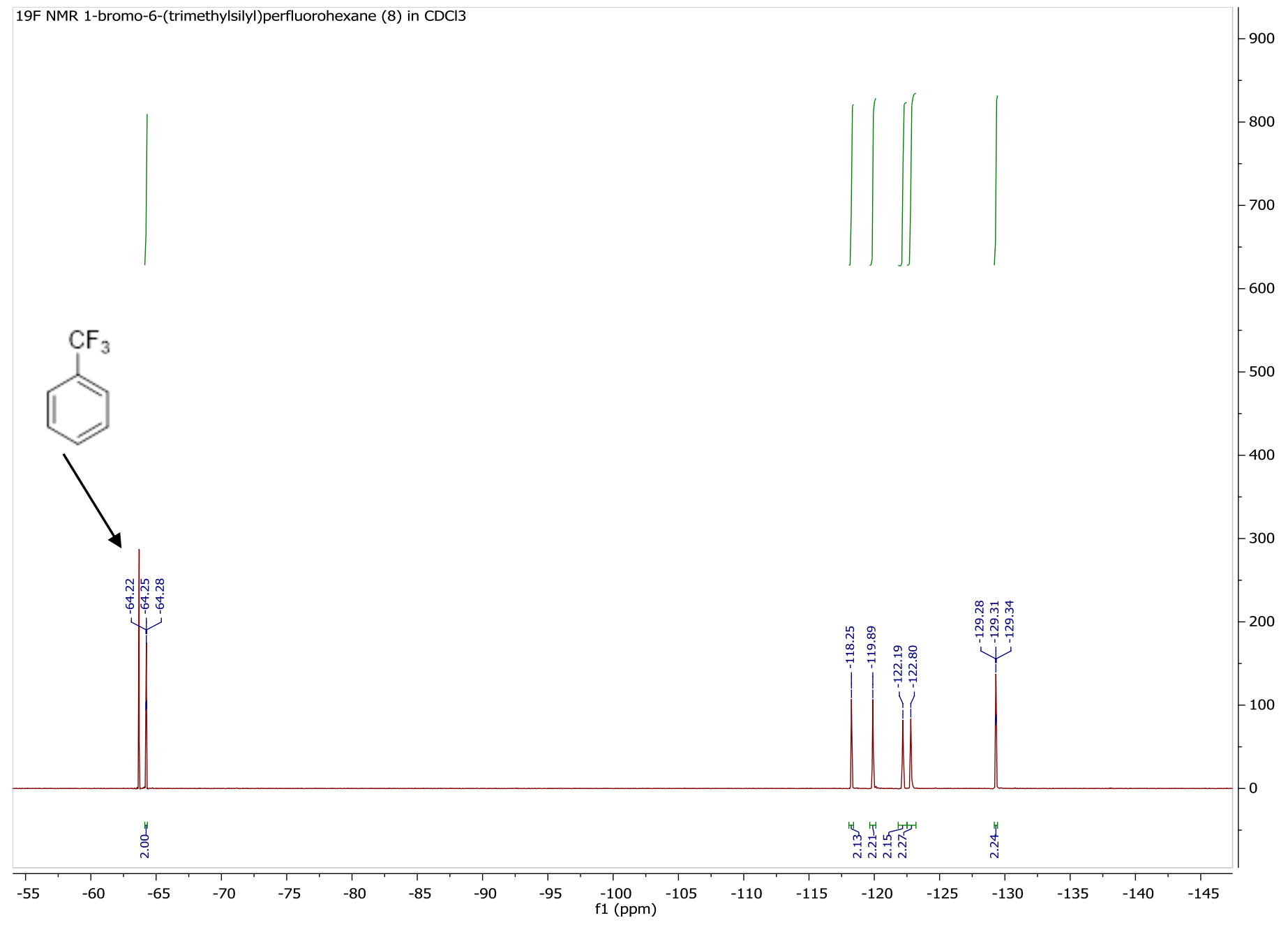


Figure 59: ${ }^{1} \mathrm{H}$ NMR spectrum of

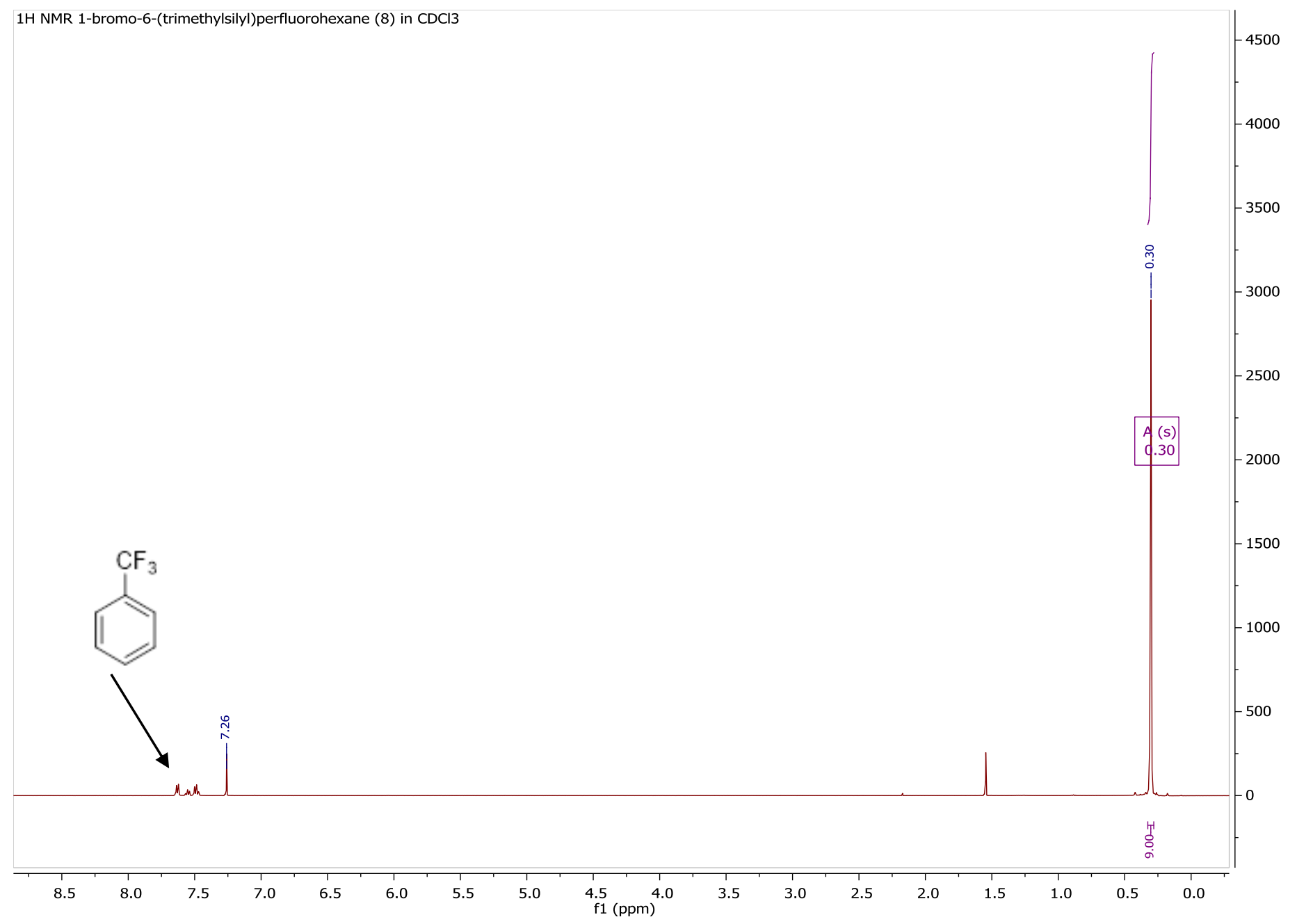


Figure S10: ${ }^{19} \mathrm{~F}$ NMR spectrum of

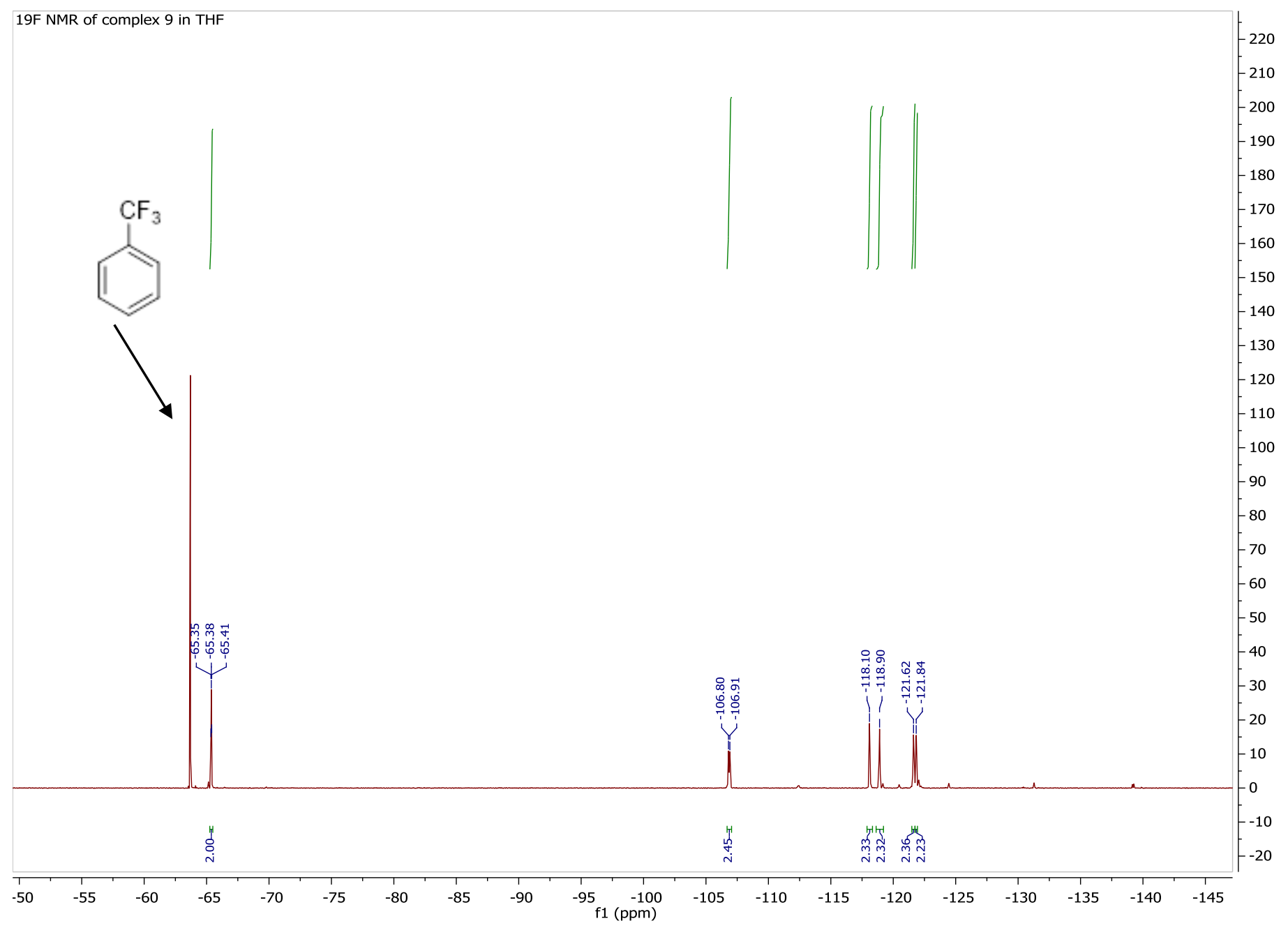


Figure S11: ${ }^{1} \mathrm{H}$ NMR spectrum of

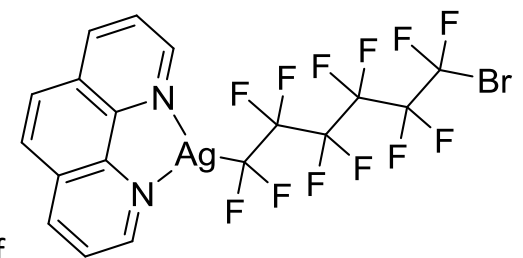

(9) in THF-D : $_{8}$

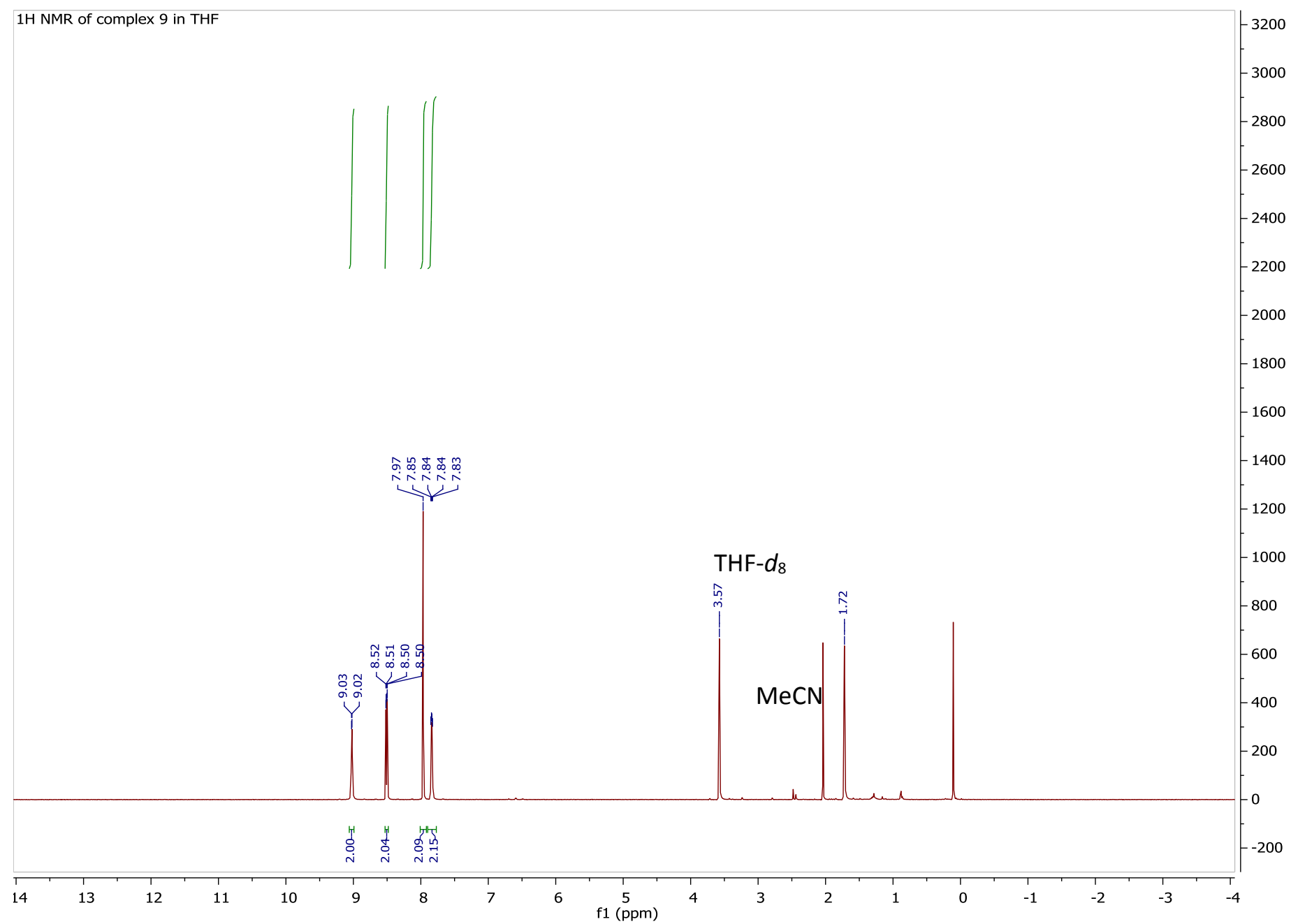


Figure S12: ${ }^{19} \mathrm{~F}$ NMR spectrum of

$19 F$ NMR of 2-(4-bromo-1,1,2,2,3,3,4,4-octafluorobutyl)pyridine in $\mathrm{CDCl} 3$

(7a) in $\mathrm{CDCl}_{3}$ :

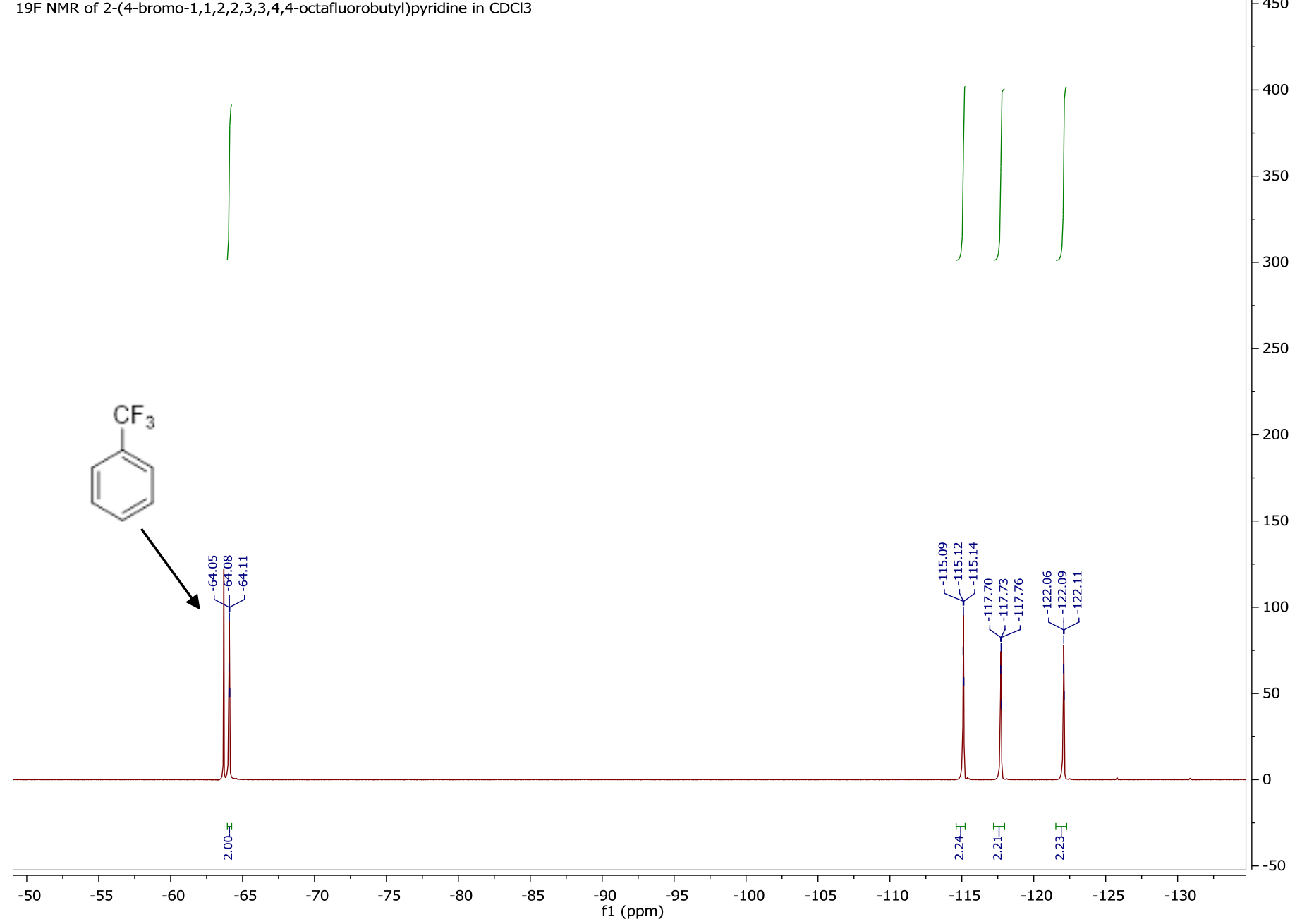


Figure 513: ${ }^{1} \mathrm{H}$ NMR spectrum of

$1 \mathrm{H}$ NMR of 2-(4-bromo-1,1,2,2,3,3,4,4-octafluorobutyl)pyridine in $\mathrm{CDCl} 3$

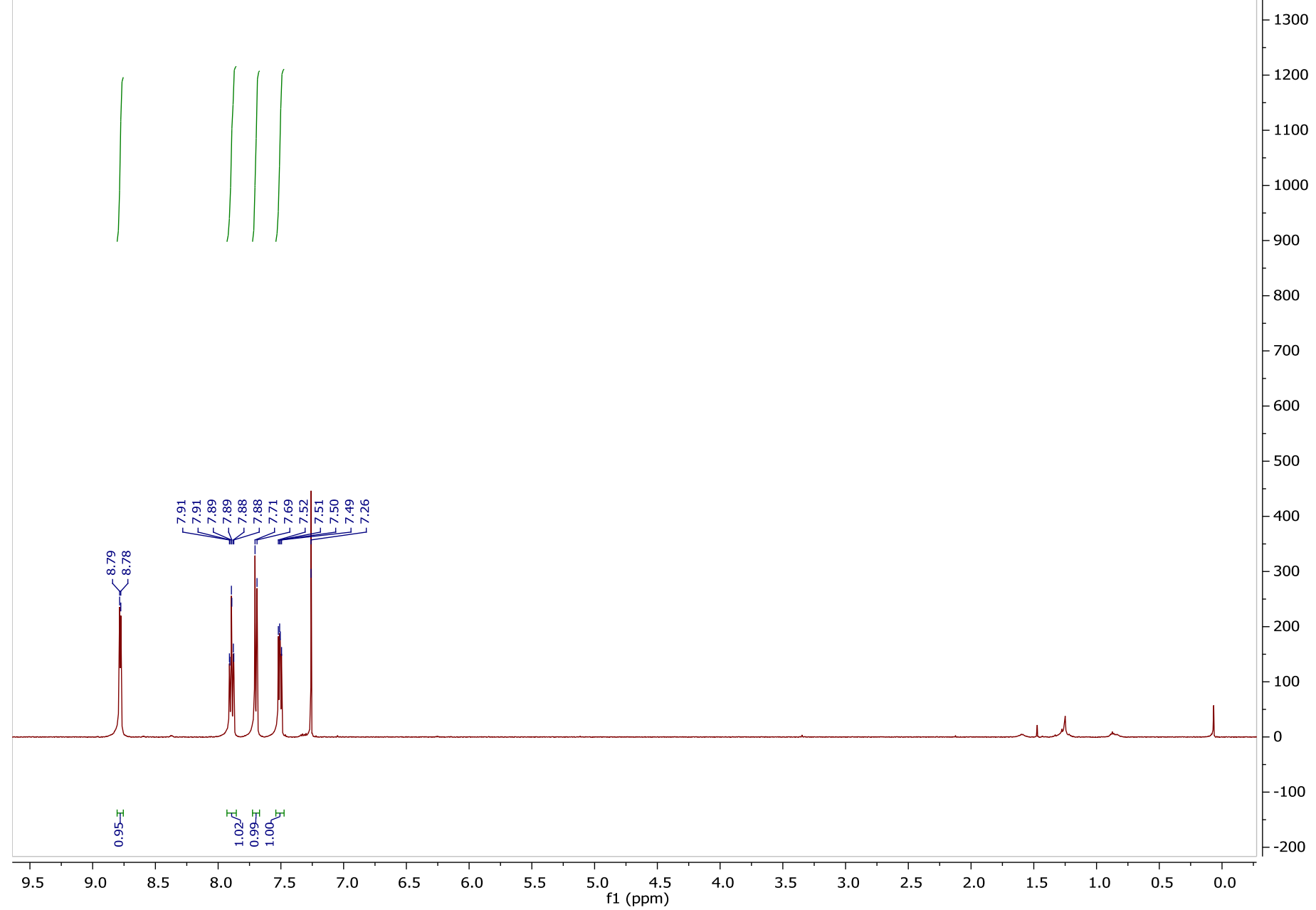


Figure S14: ${ }^{19} \mathrm{~F}$ NMR spectrum of<smiles>FC(F)(Br)C(F)(F)C(F)(F)C(F)(F)c1cccnc1</smiles>

19F NMR of 3-(4-bromo-1,1,2,2,3,3,4,4-octafluorobutyl)pyridine in MeCN

(7b) in $\mathrm{MeCN}-d_{3}$ :

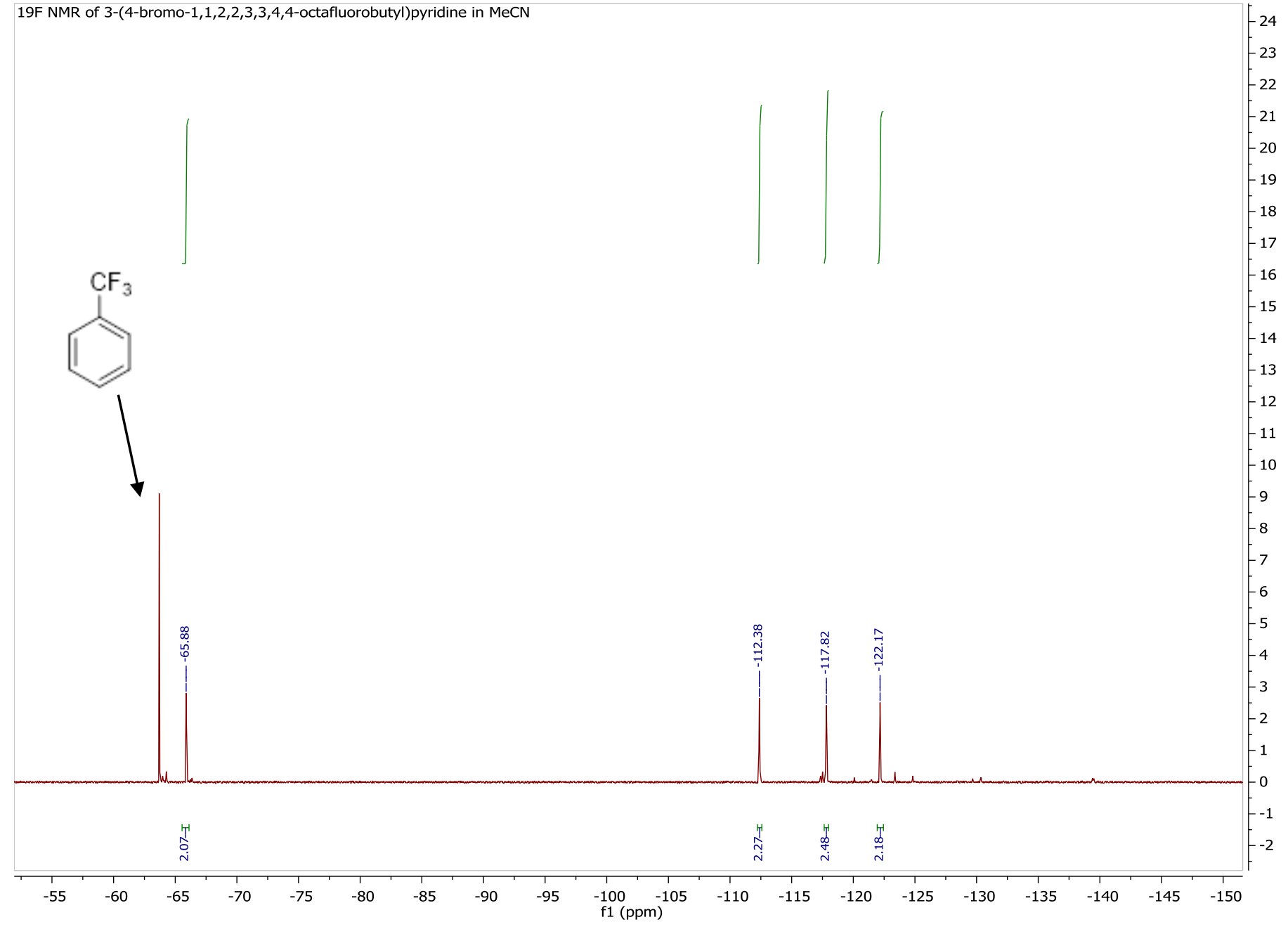




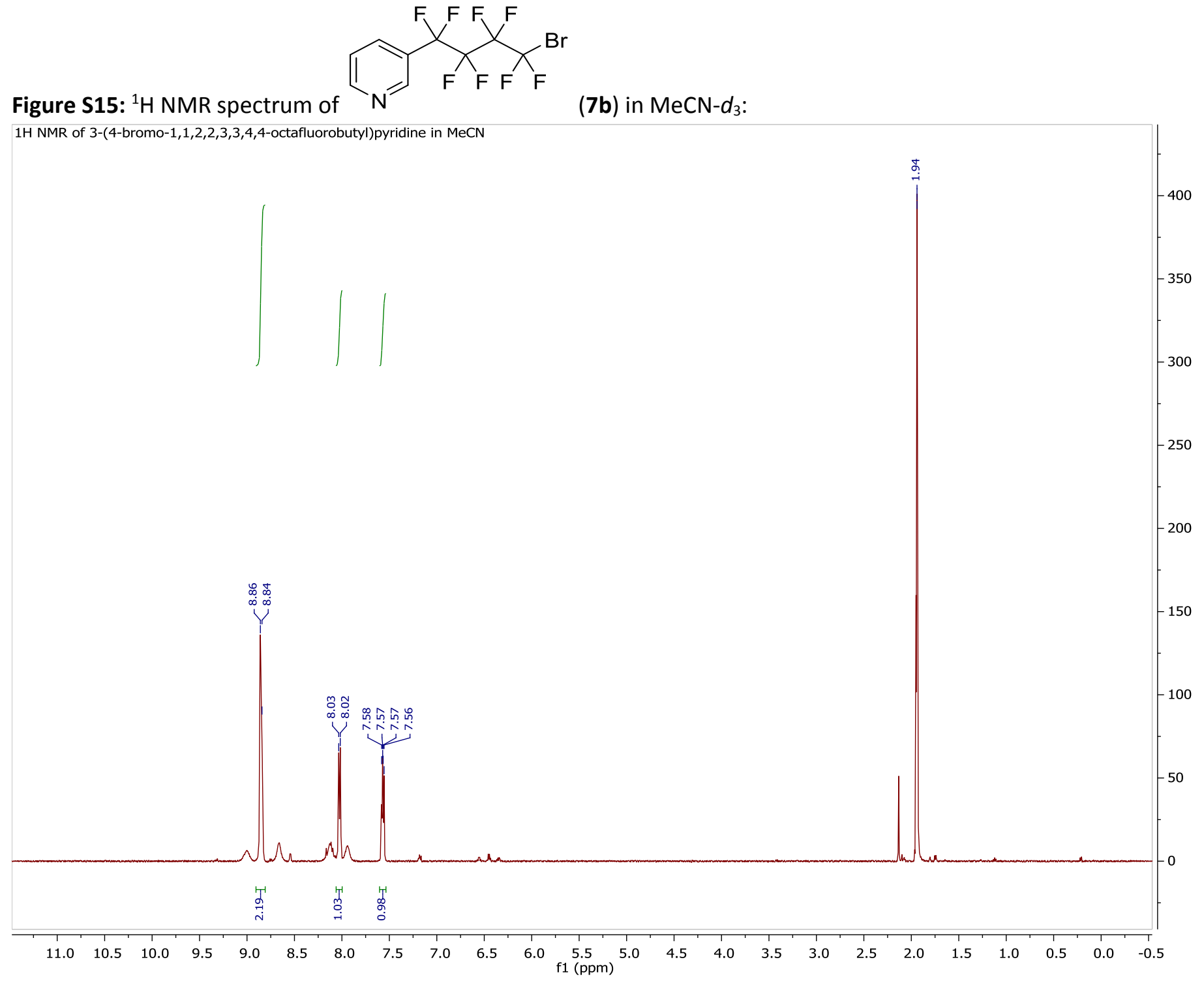




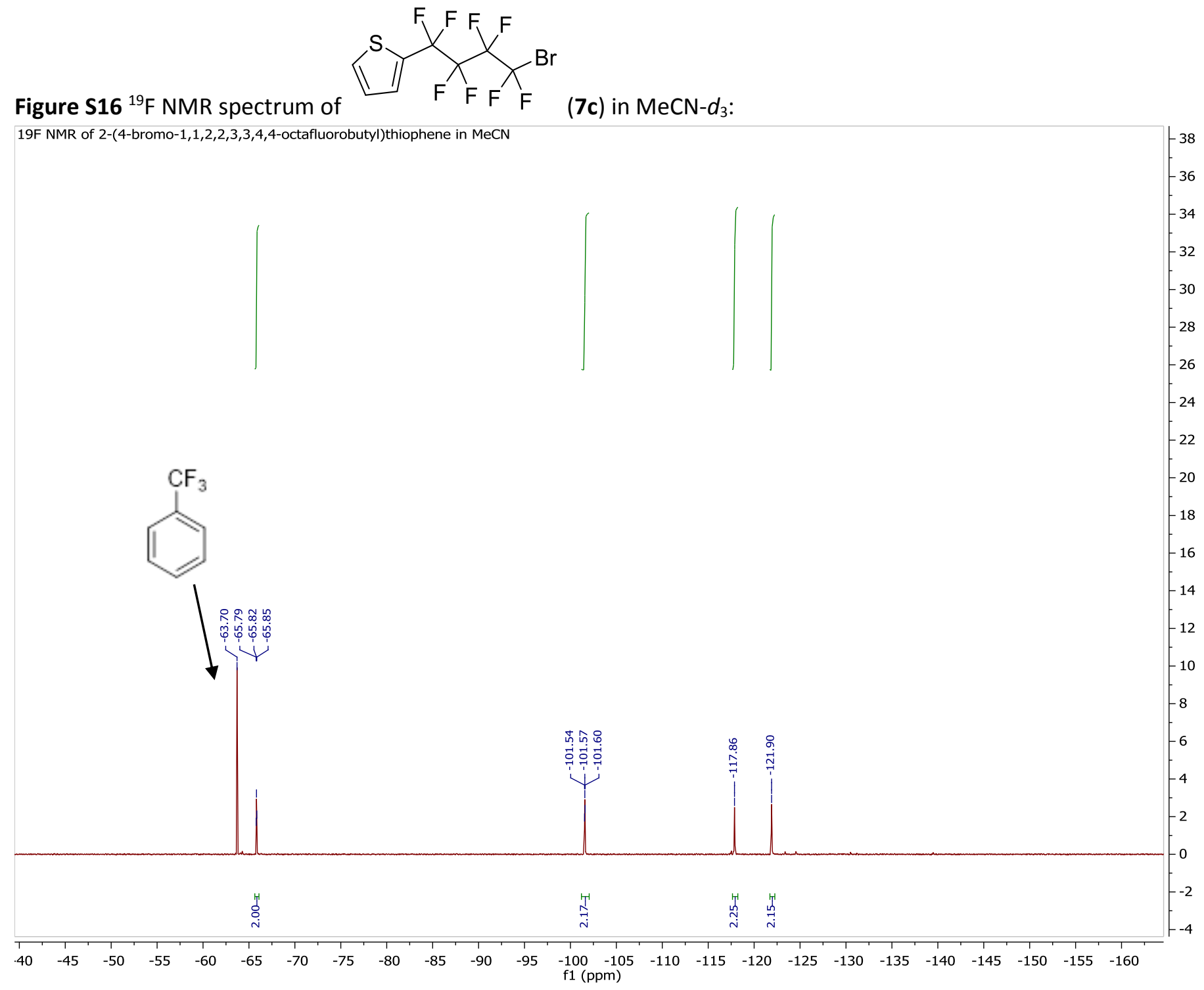




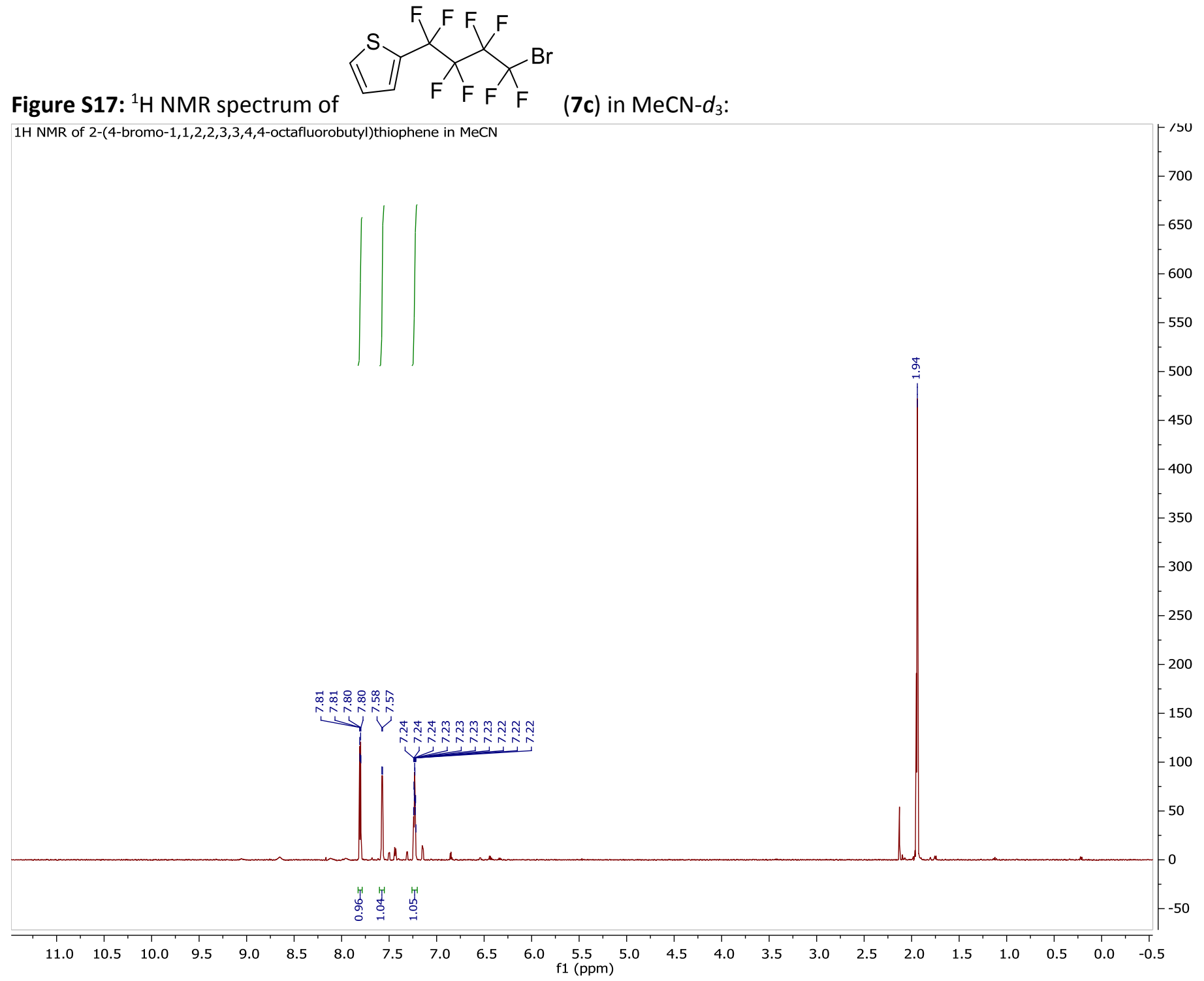




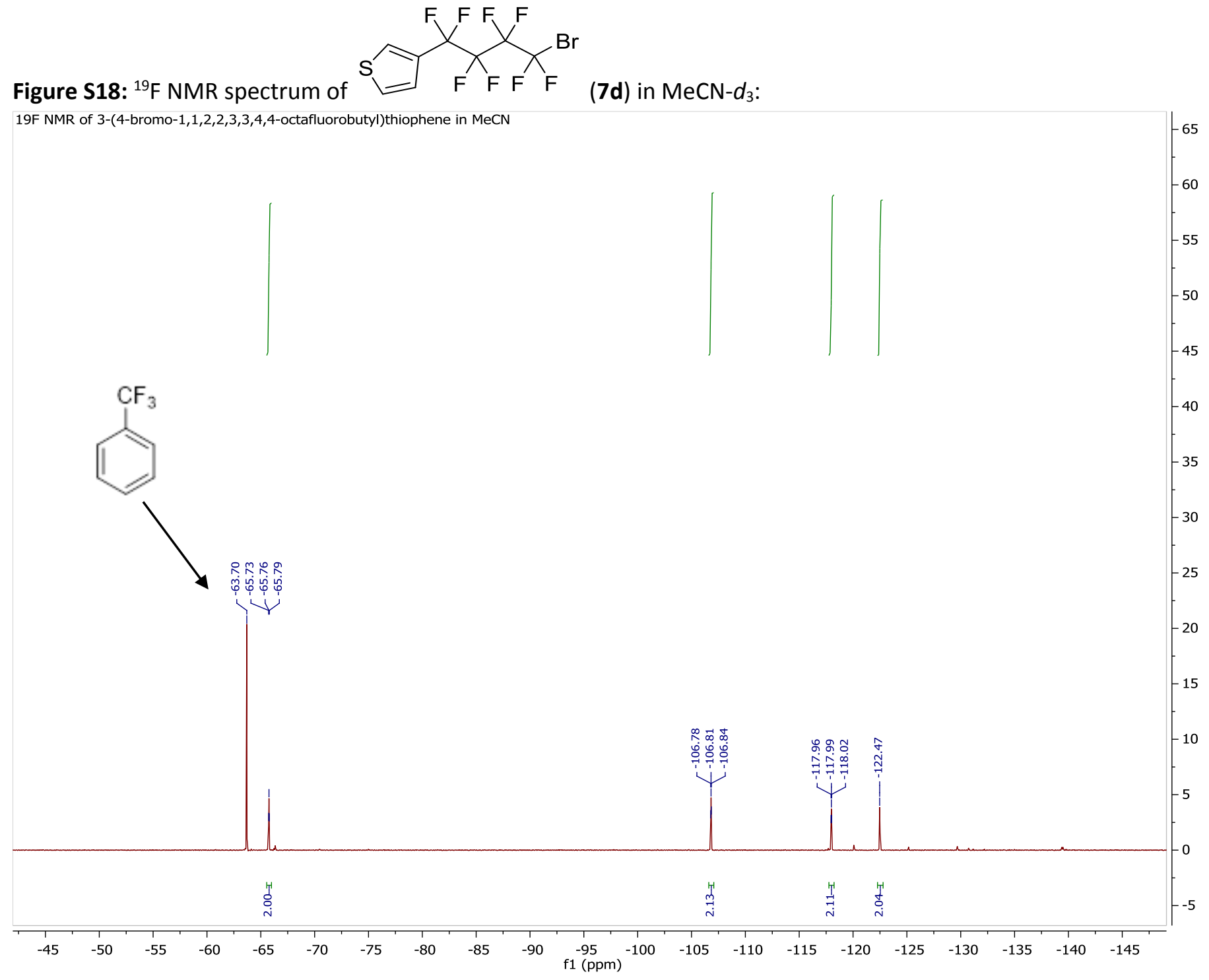


Figure S19: ${ }^{1} \mathrm{H}$ NMR spectrum of

1H NMR of 3-(4-bromo-1,1,2,2,3,3,4,4-octafluorobutyl)thiophene in MeCN

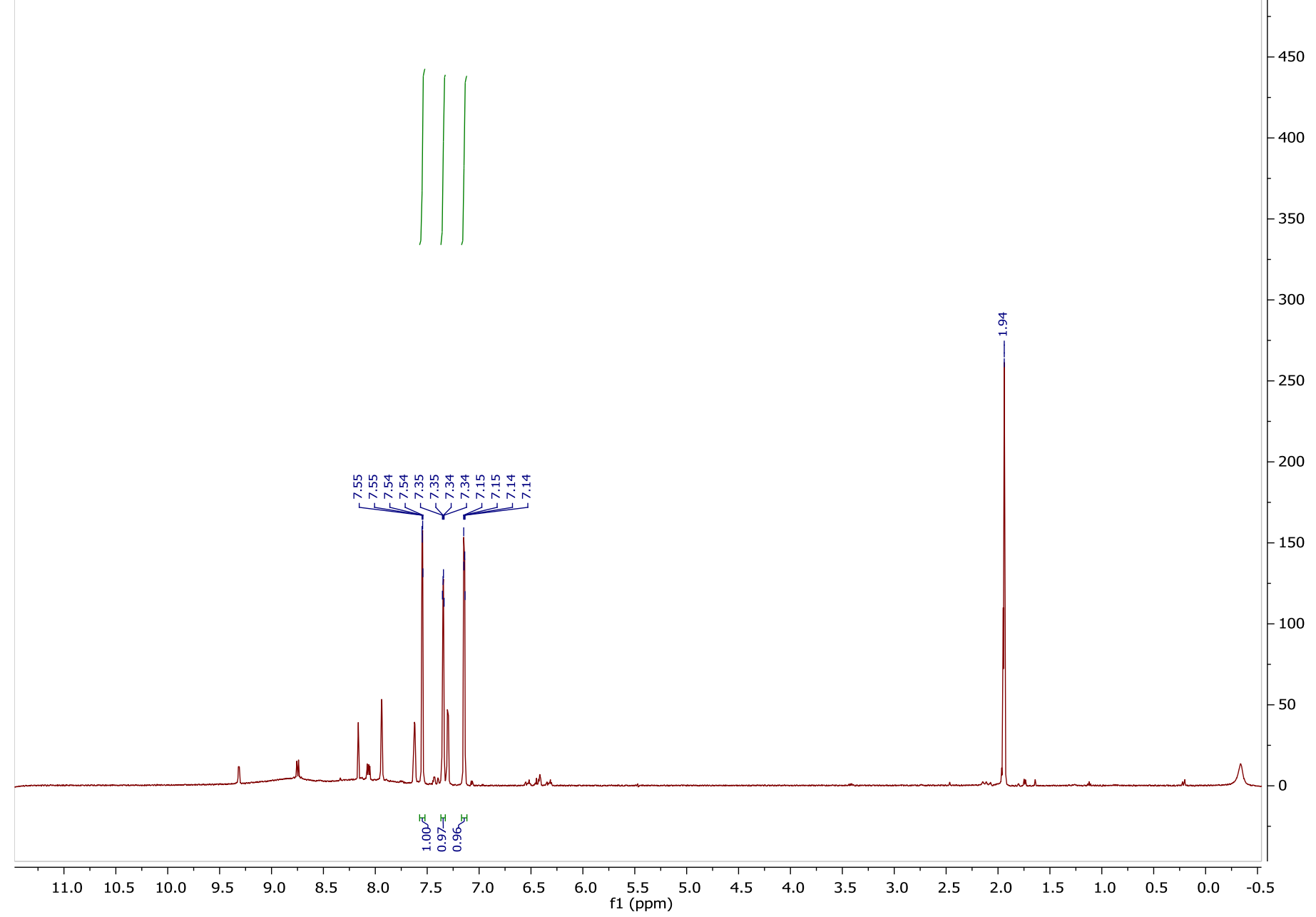


Figure S20: ${ }^{19} \mathrm{~F}$ NMR spectrum of<smiles>FC(F)(Br)C(F)(F)C(F)(F)C(F)(F)c1ccnc2cc(Cl)ccc12</smiles>

$19 F$ NMR of 7-chloro-4-(4-bromo-1,1,2,2,3,3,4,4-octafluorobutyl)quinoline in $\mathrm{CDCl} 3$

(7e) in $\mathrm{CDCl}_{3}$

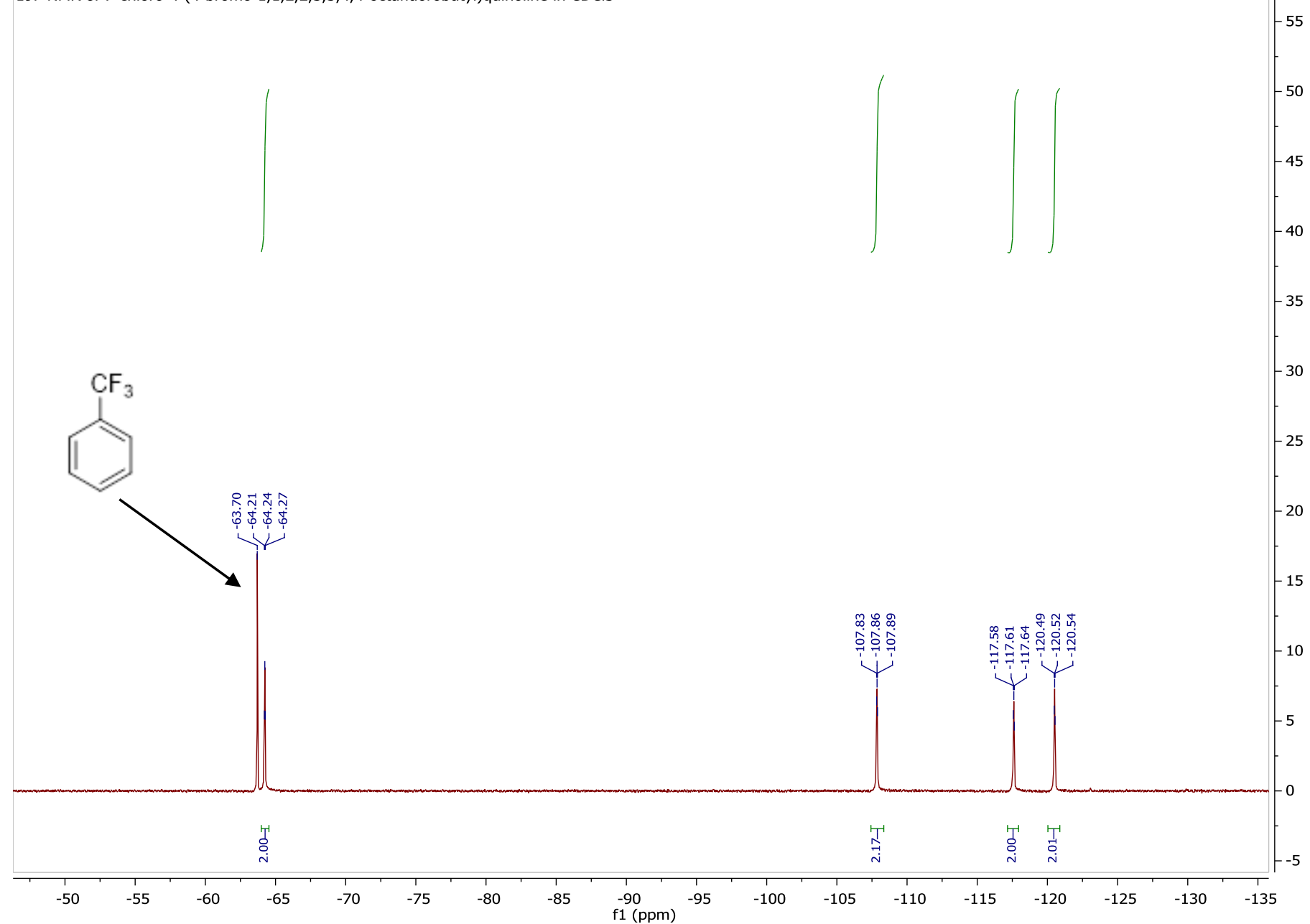


Figure S21: ${ }^{1} \mathrm{H}$ NMR spectrum of<smiles>FC(F)(Br)C(F)(F)C(F)(F)C(F)(F)c1ccnc2cc(Cl)ccc12</smiles>

(7e) in $\mathrm{CDCl}_{3}$ :

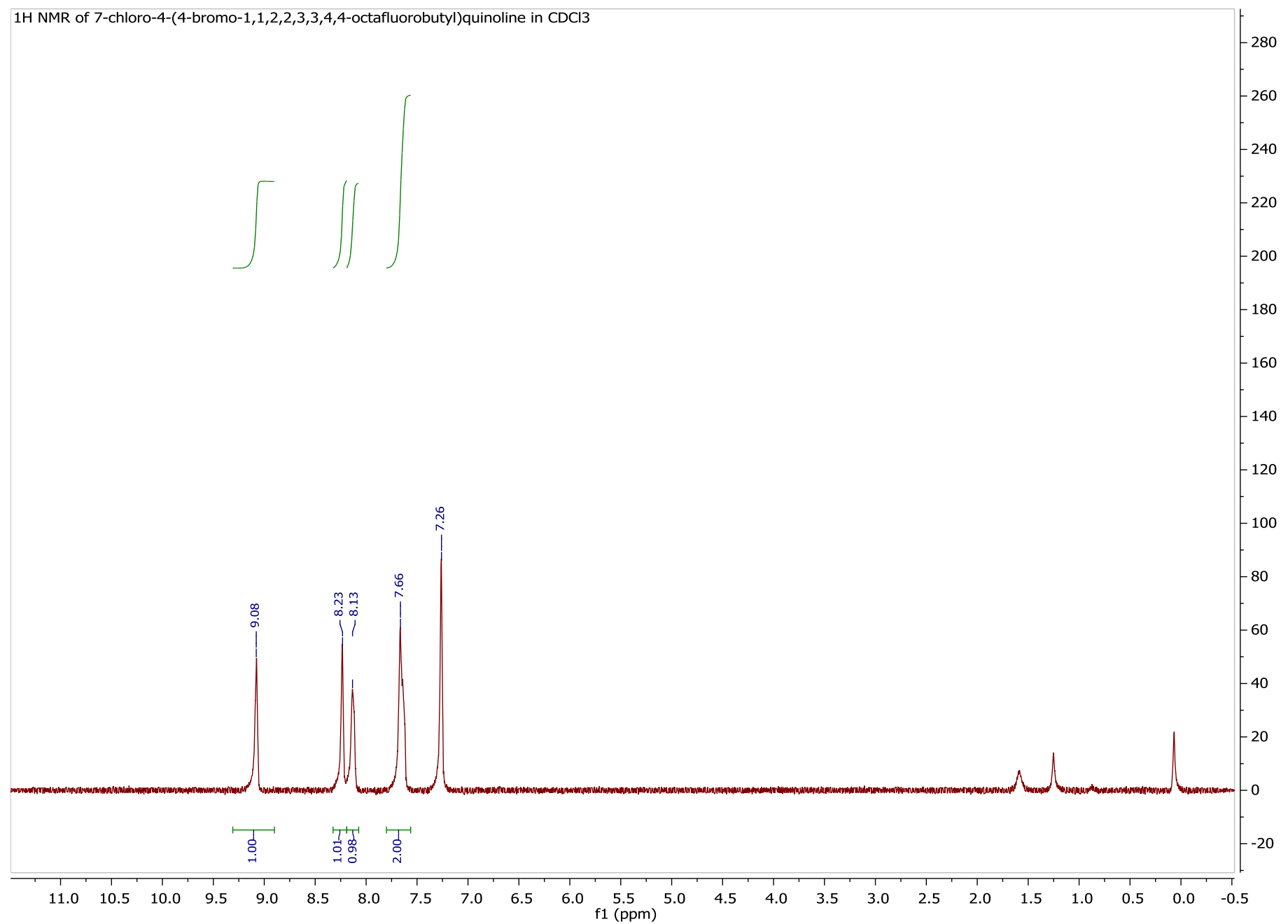


Figure S22: ${ }^{19} \mathrm{~F}$ NMR spectrum of<smiles>FC(F)(Br)C(F)(F)C(F)(F)C(F)(F)c1ccccc1</smiles>

(7f) in $\mathrm{MeCN}-d_{3}$ :

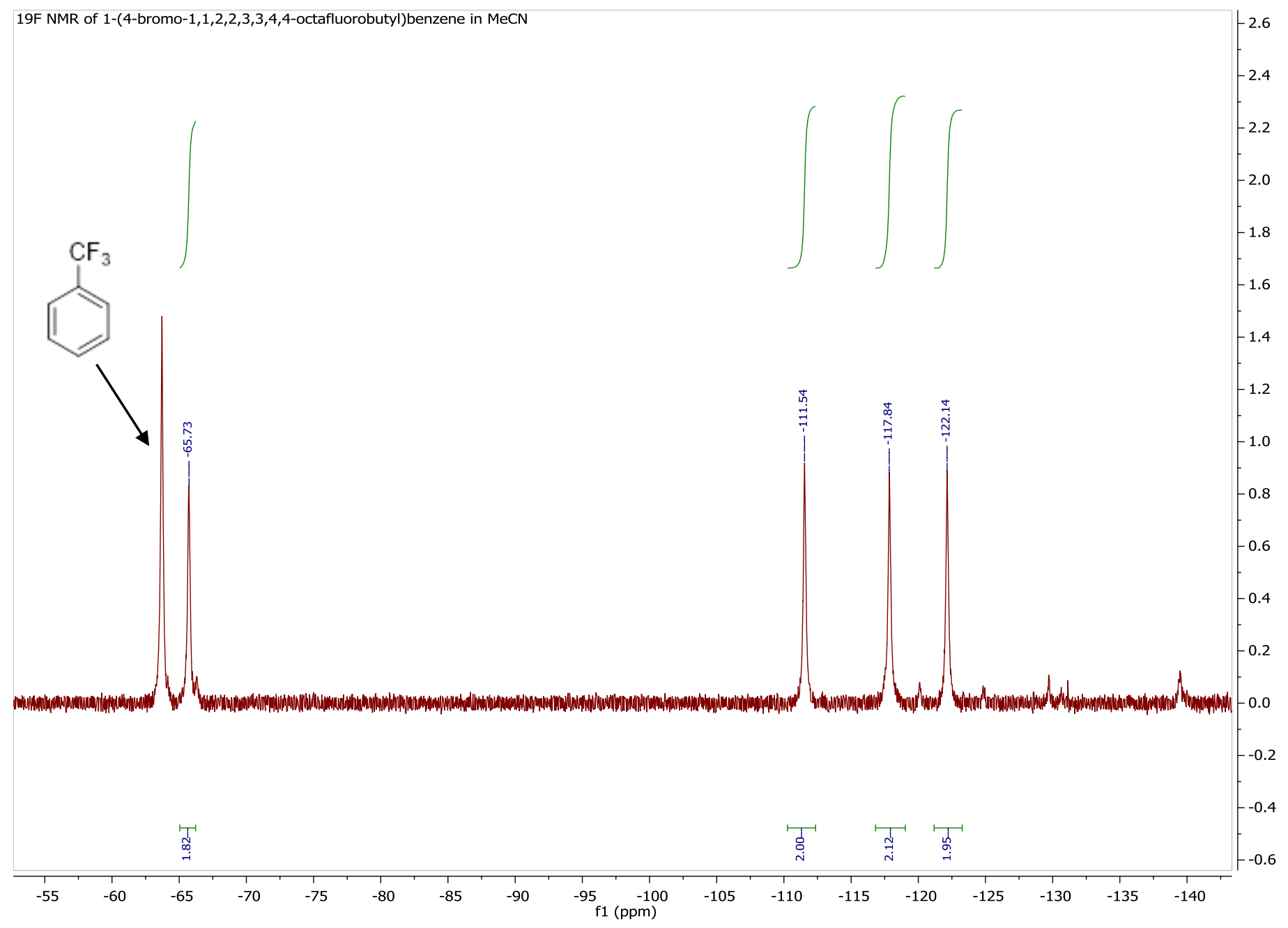


Figure S23: ${ }^{1} \mathrm{H}$ NMR spectrum of

$1 \mathrm{H}$ NMR of 1-(4-bromo-1,1,2,2,3,3,4,4-octafluorobutyl)benzene in MeCN

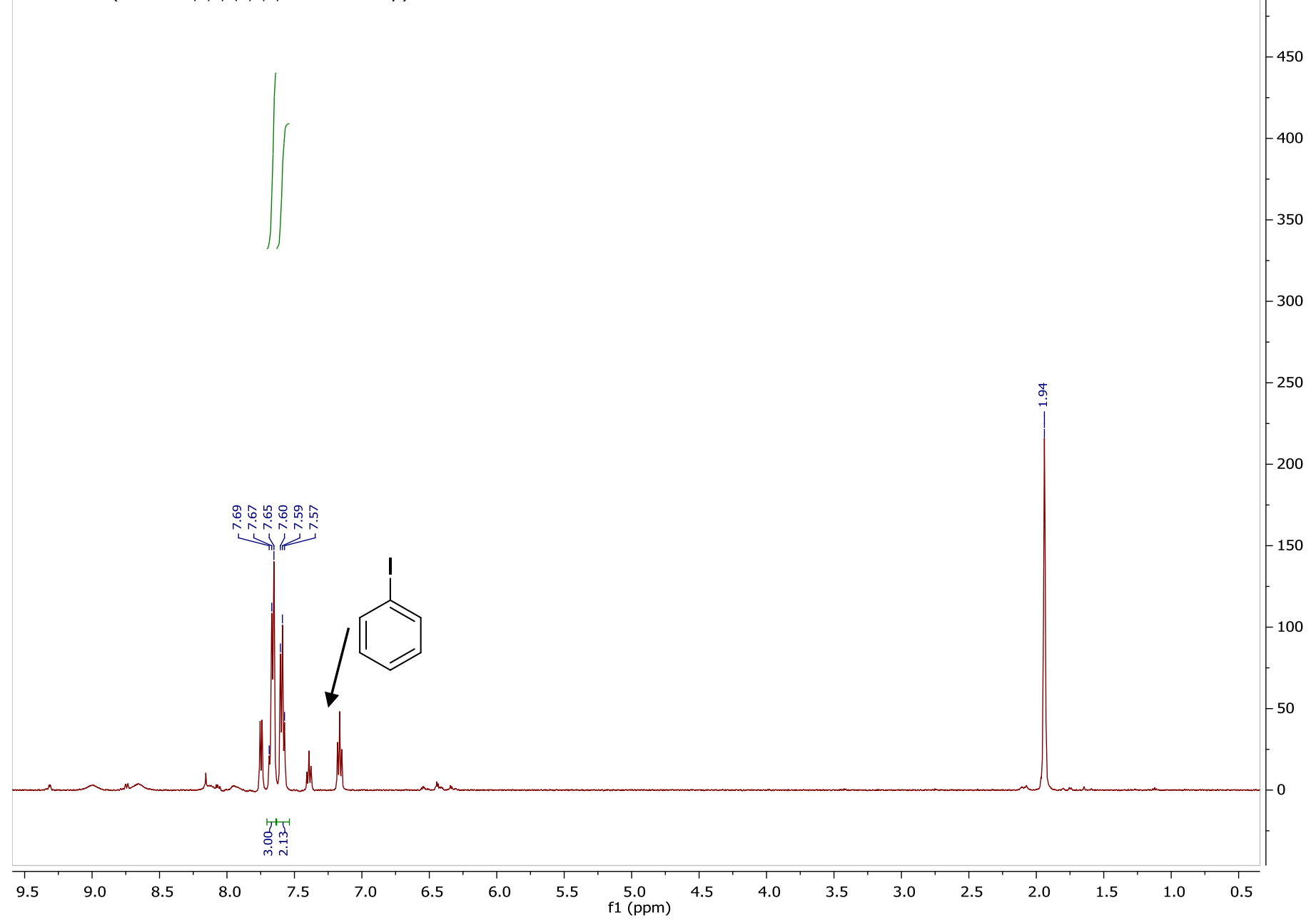




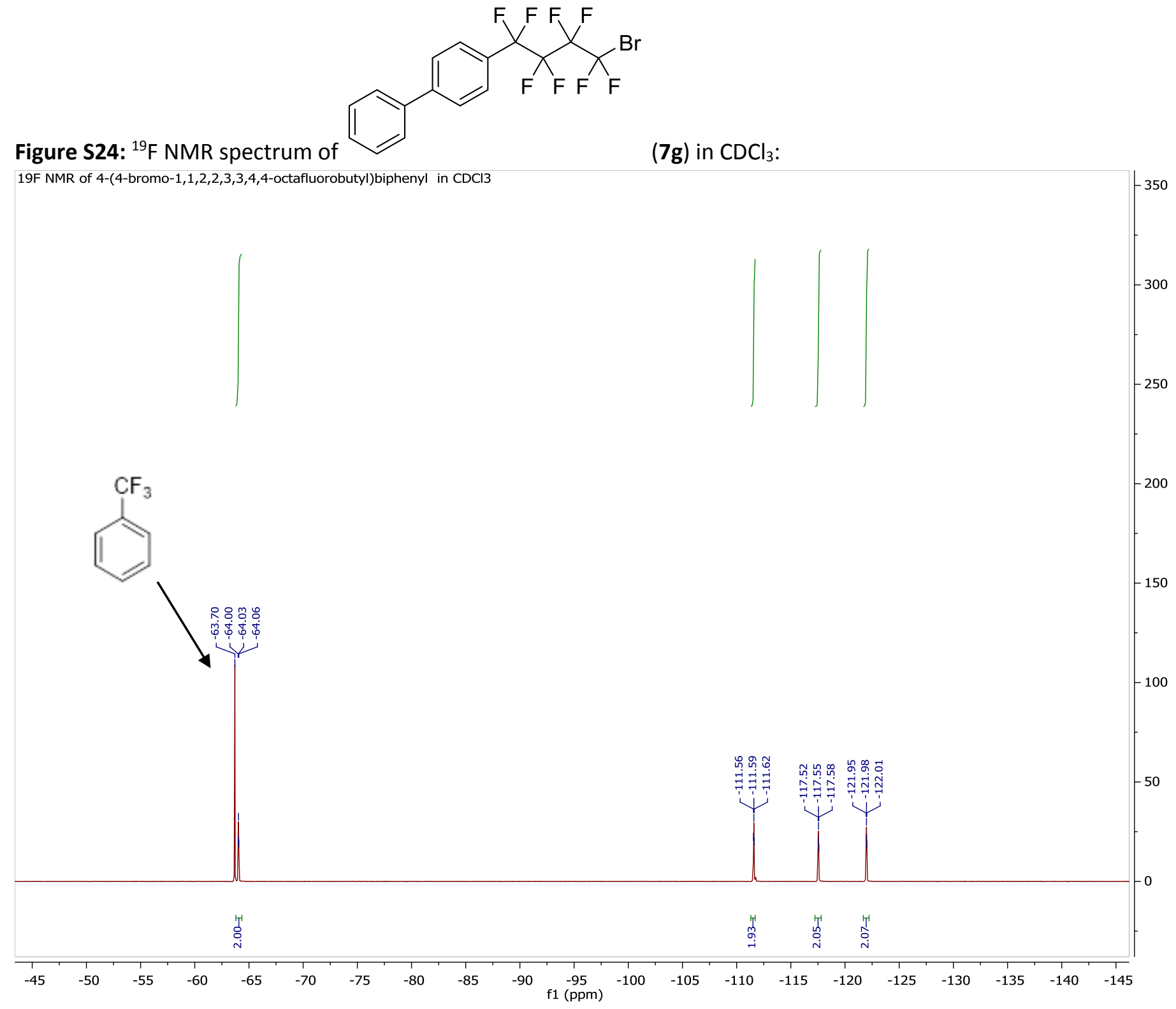


Figure S25: ${ }^{1} \mathrm{H}$ NMR spectrum of<smiles>FC(F)(Br)C(F)(F)C(F)(F)C(F)(F)c1ccc(-c2ccc(I)cc2)cc1</smiles>

$1 \mathrm{H}$ NMR of 4-(4-bromo-1,1,2,2,3,3,4,4-octafluorobutyl)biphenyl in CDCI3

$(7 \mathrm{~g})$ in $\mathrm{CDCl}_{3}$ :

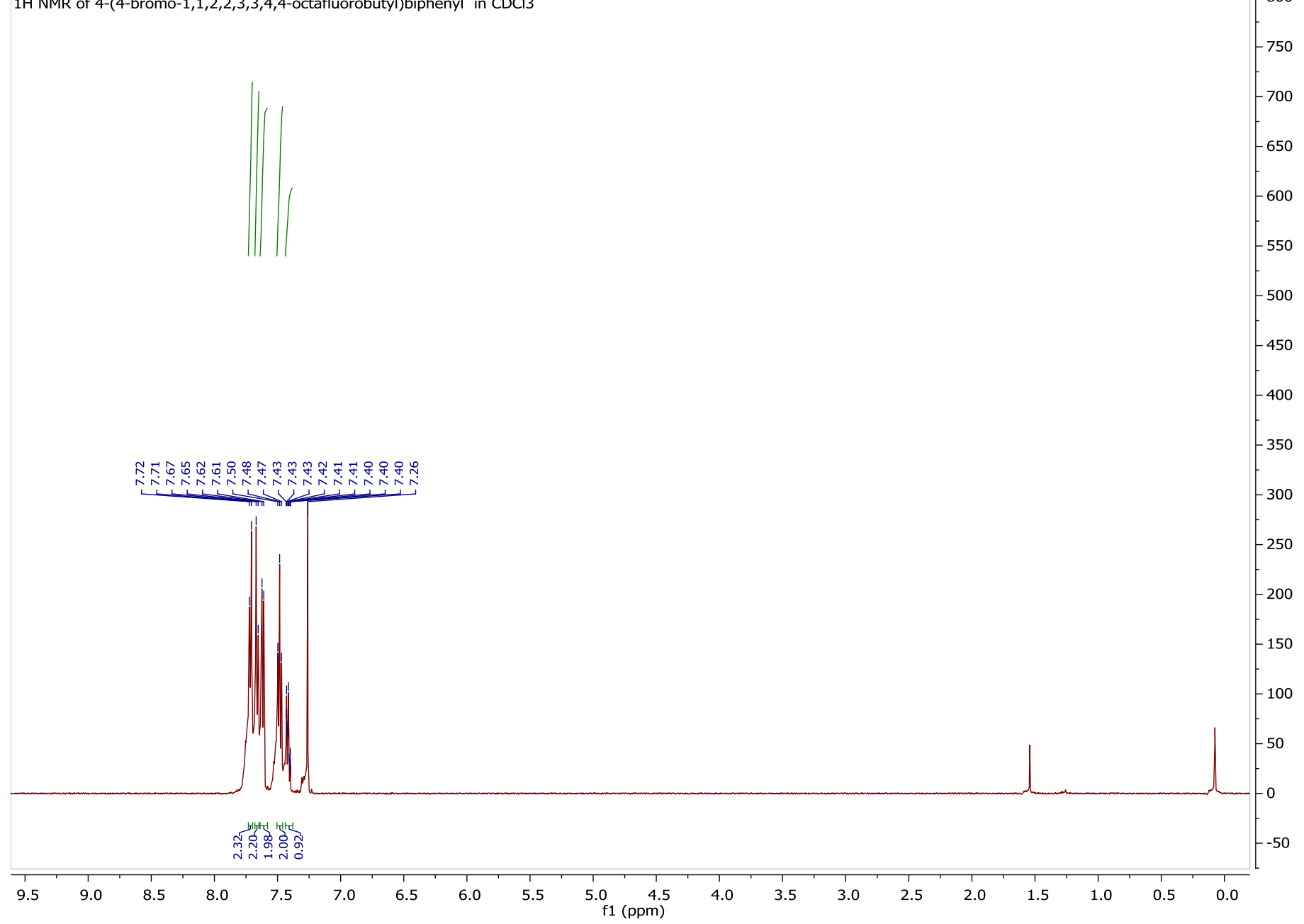




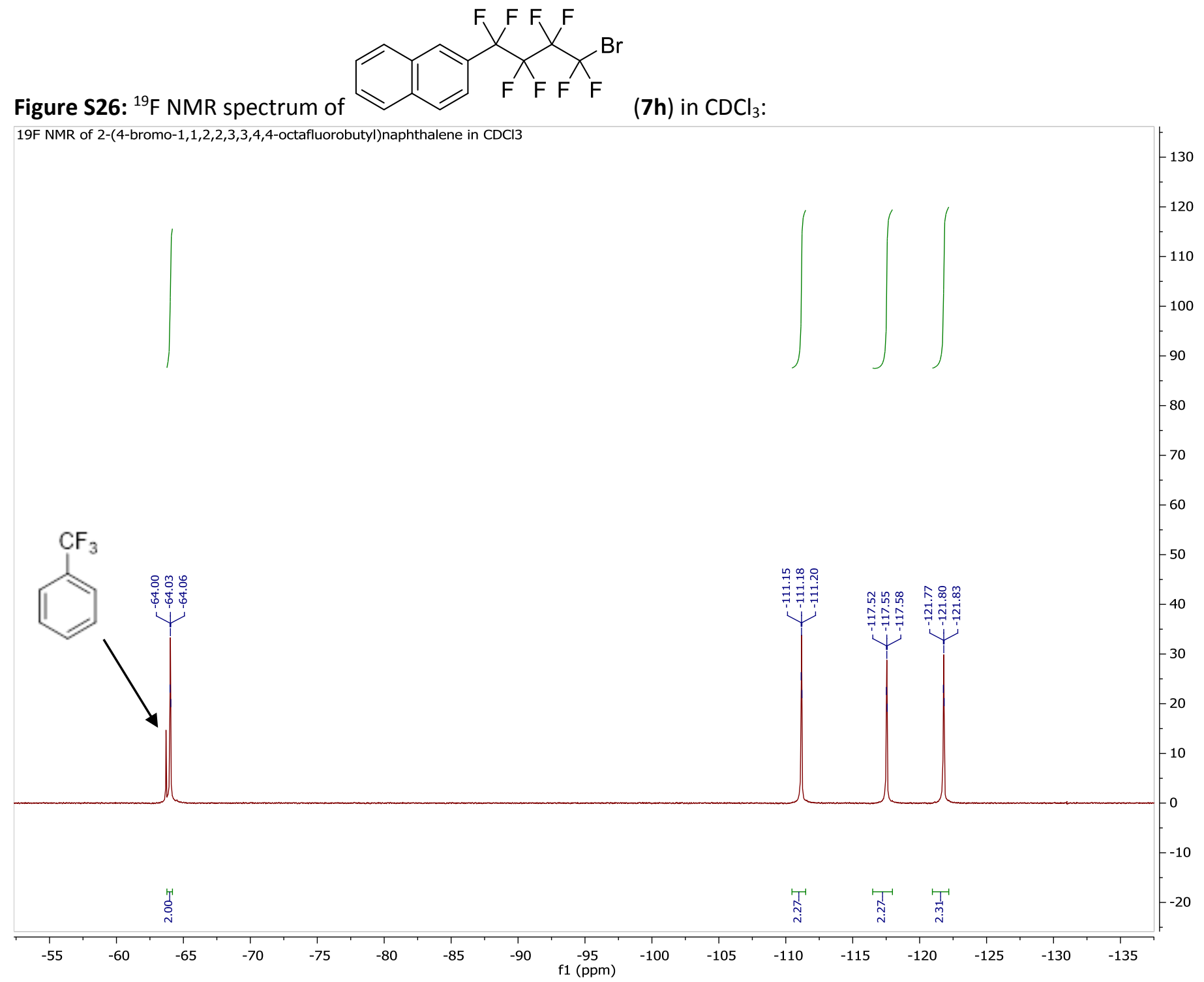


Figure S27: ${ }^{1} \mathrm{H}$ NMR spectrum of<smiles>FC(F)(Br)C(F)(F)C(F)(F)C(F)(F)c1ccc2ccccc2c1</smiles>

$1 \mathrm{H}$ NMR of 2-(4-bromo-1,1,2,2,3,3,4,4-octafluorobutyl)naphthalene in $\mathrm{CDCl} 3$

(7h) in $\mathrm{CDCl}_{3}$ :

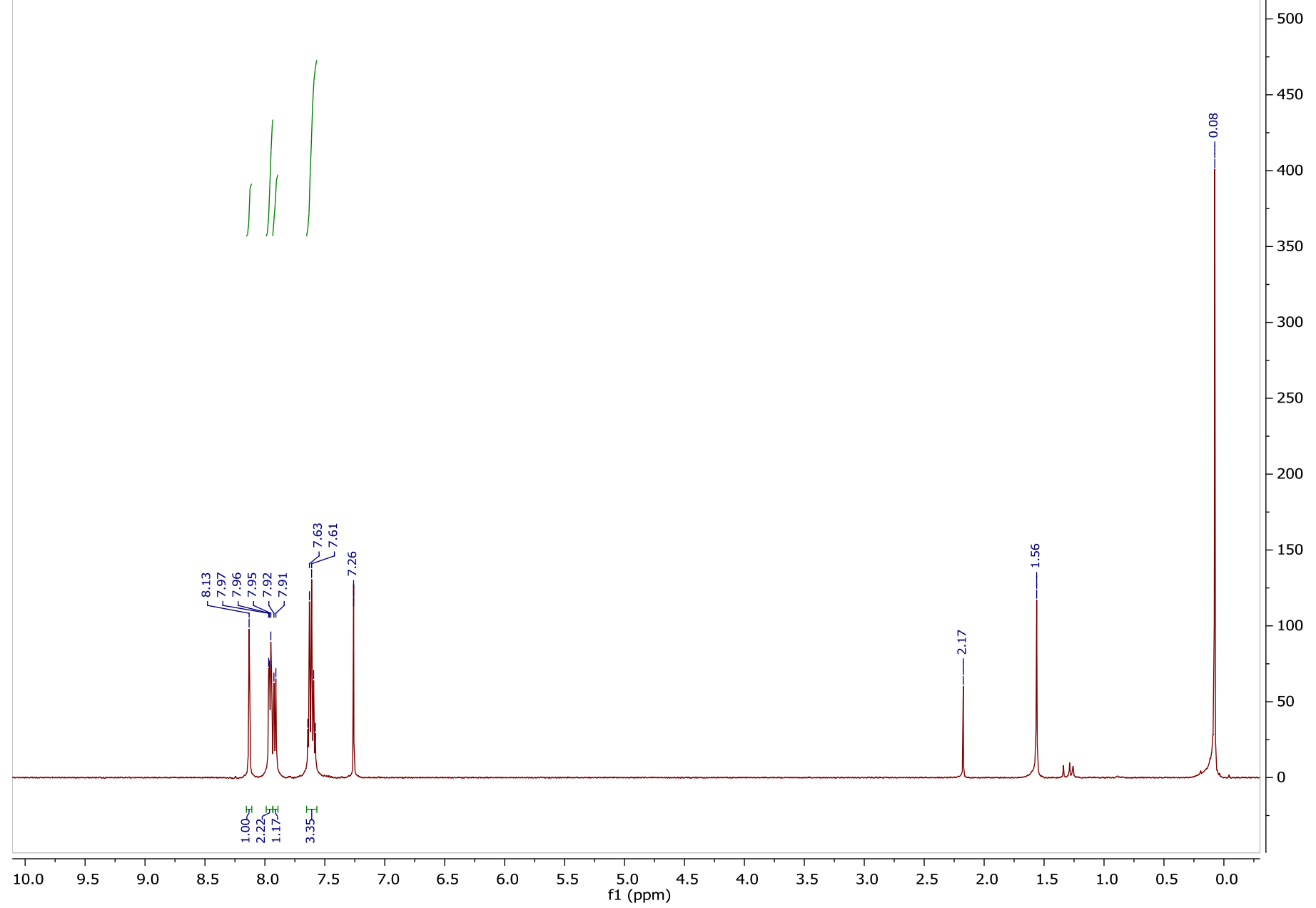


Figure S28: ${ }^{19} \mathrm{~F}$ NMR spectrum of<smiles>FC(F)(Br)C(F)(F)C(F)(F)C(F)(F)c1cccc2ccccc12</smiles>

$19 F$ NMR of 1-(4-bromo-1,1,2,2,3,3,4,4-octafluorobuty)naphthalene in CDCl3

(7i) in $\mathrm{CDCl}_{3}$ :

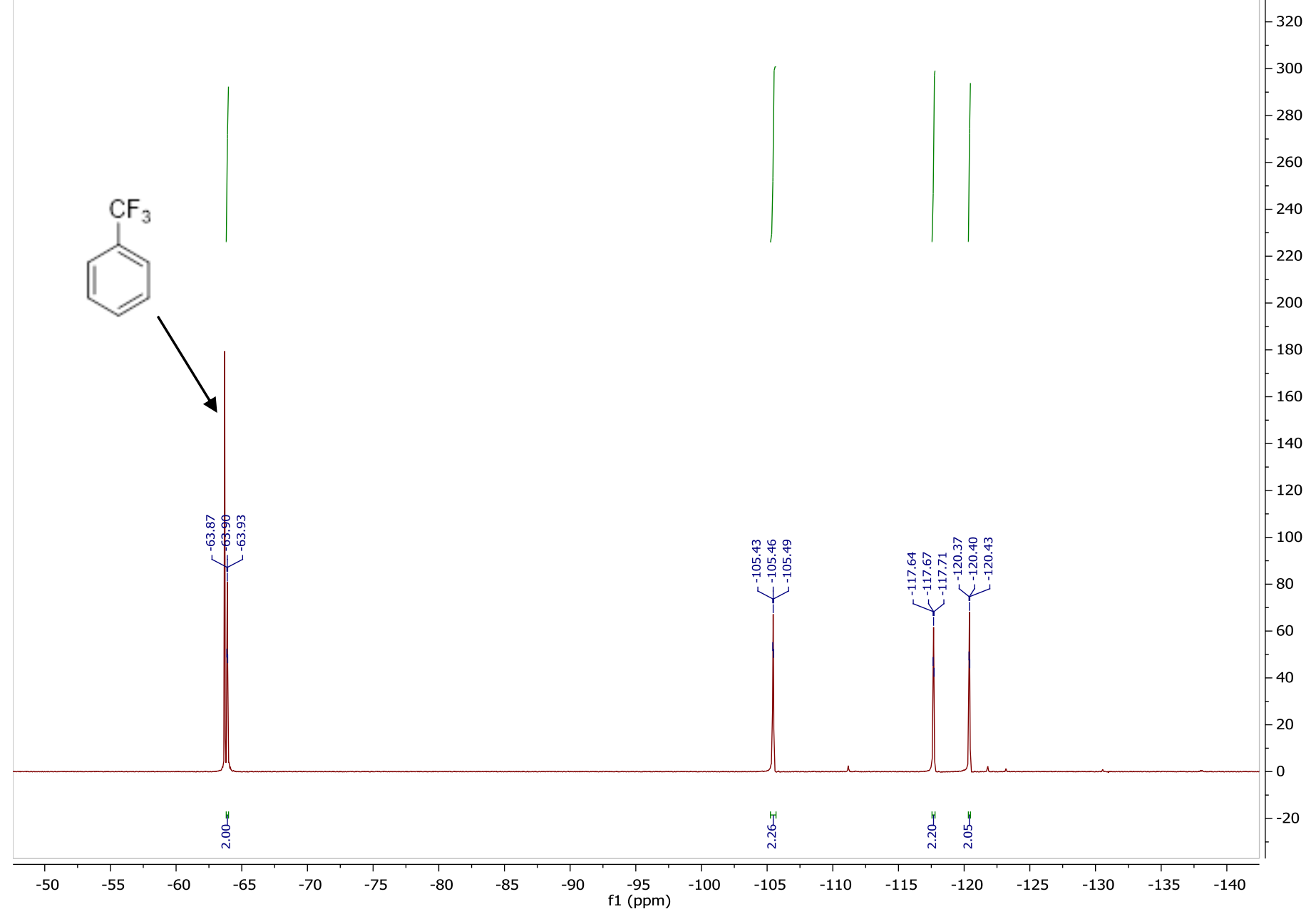


Figure S29: ${ }^{1} \mathrm{H}$ NMR spectrum of<smiles>FC(F)(Br)C(F)(F)C(F)(F)C(F)(F)c1cccc2ccccc12</smiles>

1H NMR of 1-(4-bromo-1,1,2,2,3,3,4,4-octafluorobutyl)naphthalene in $\mathrm{CDCl} 3$

(7i) in $\mathrm{CDCl}_{3}$ :

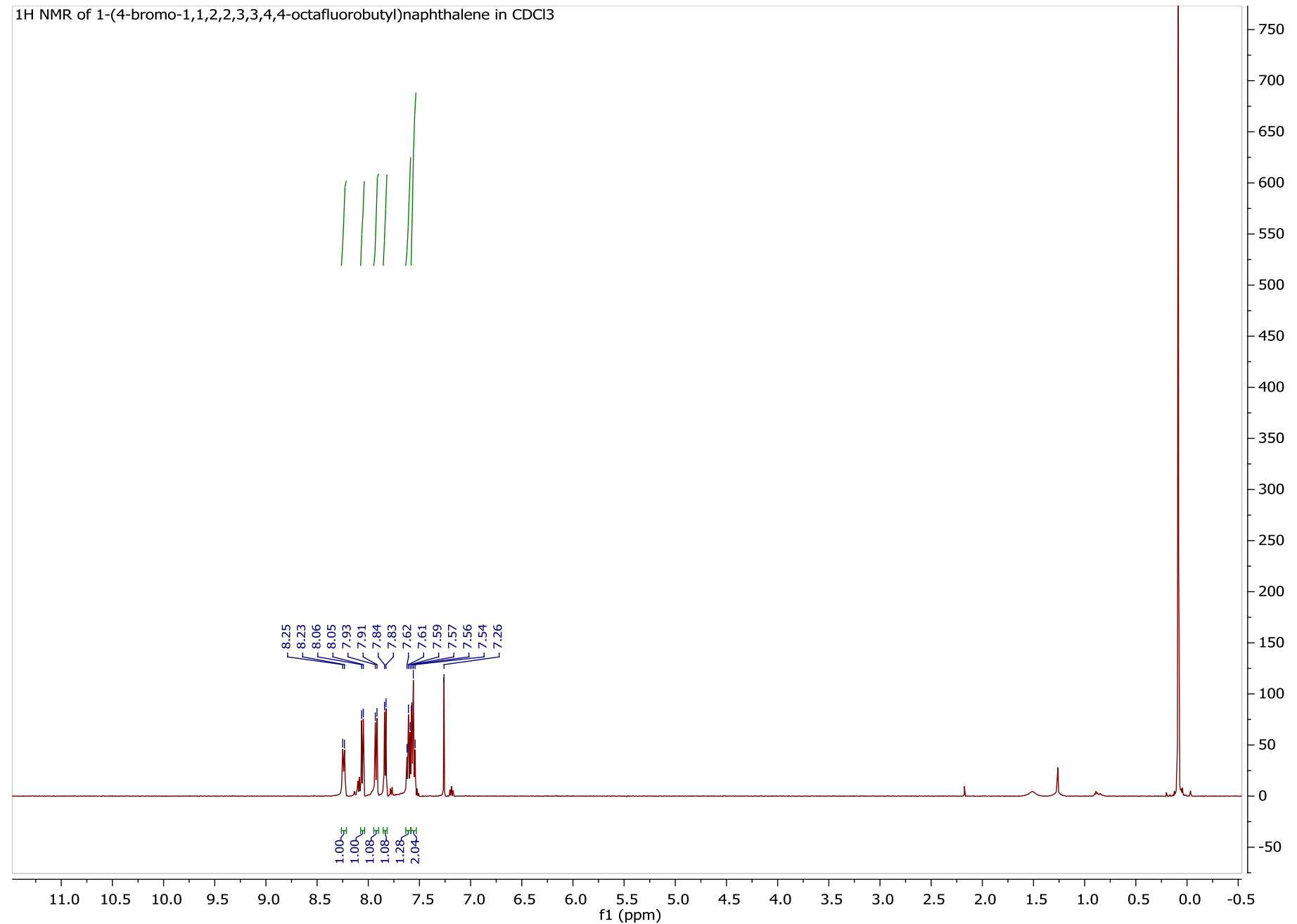




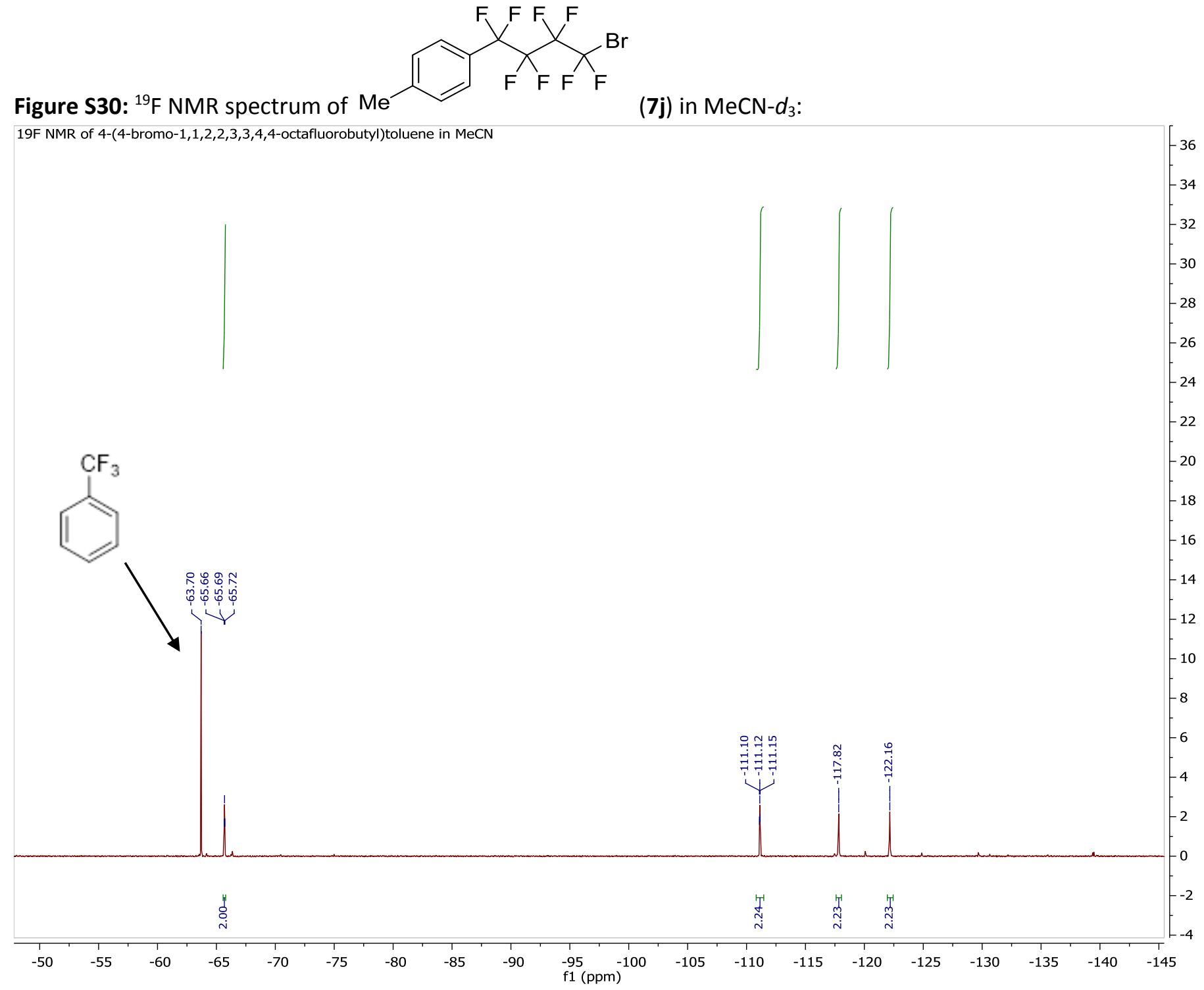




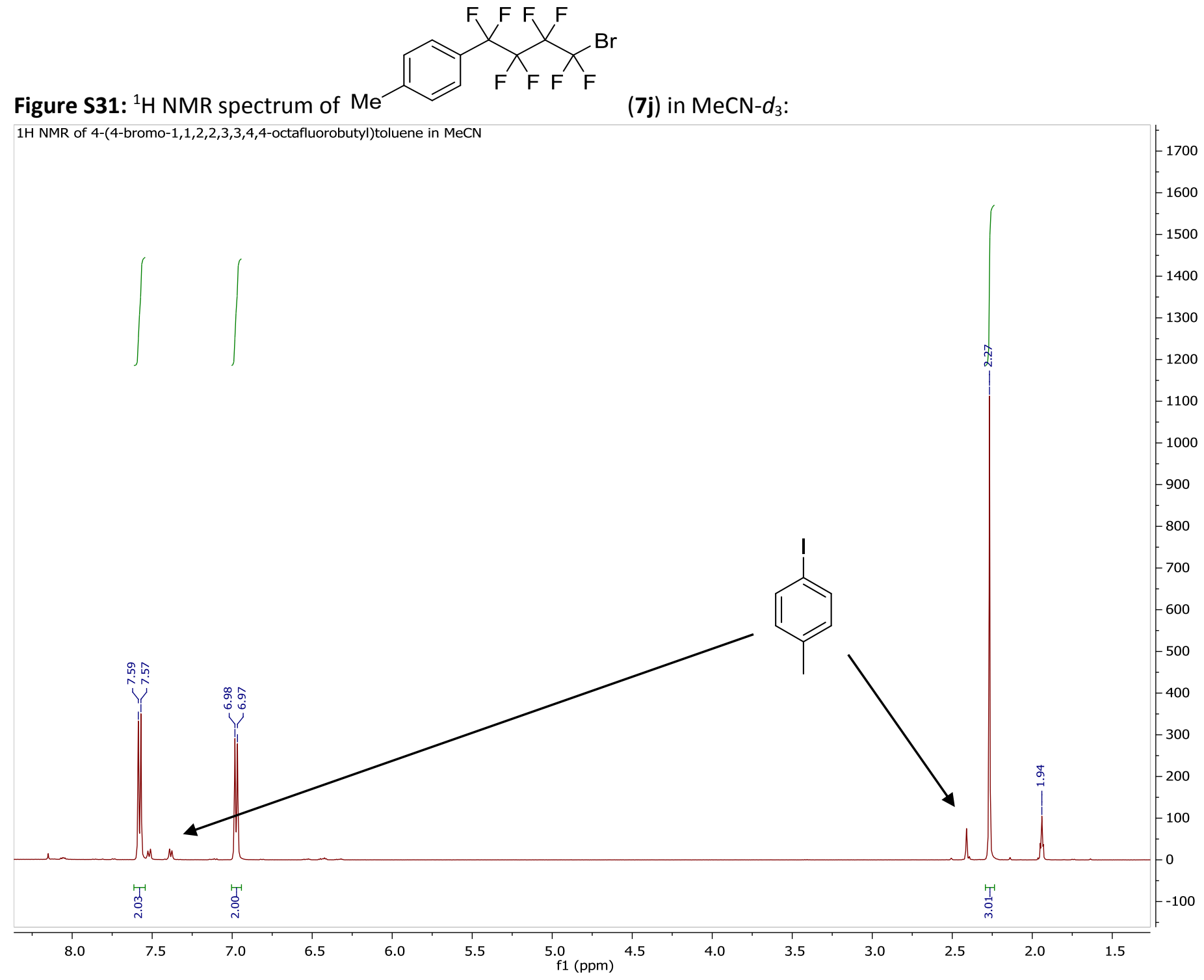




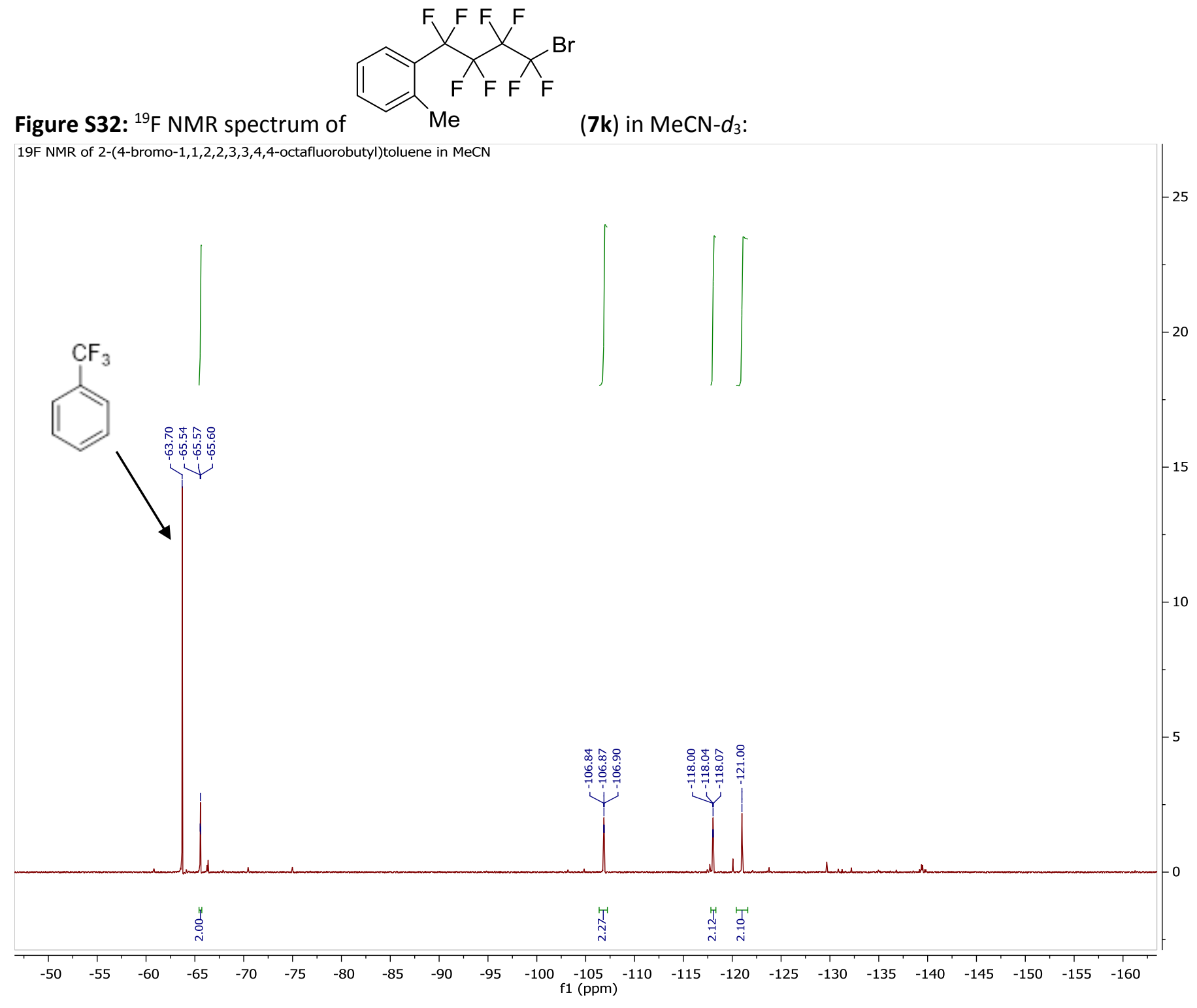


Figure S33: ${ }^{1} \mathrm{H}$ NMR spectrum of<smiles>Cc1ccccc1C(F)(F)C(F)(F)C(F)(F)C(F)(F)Br</smiles>

(7k) in $\mathrm{MeCN}-d_{3}$ :

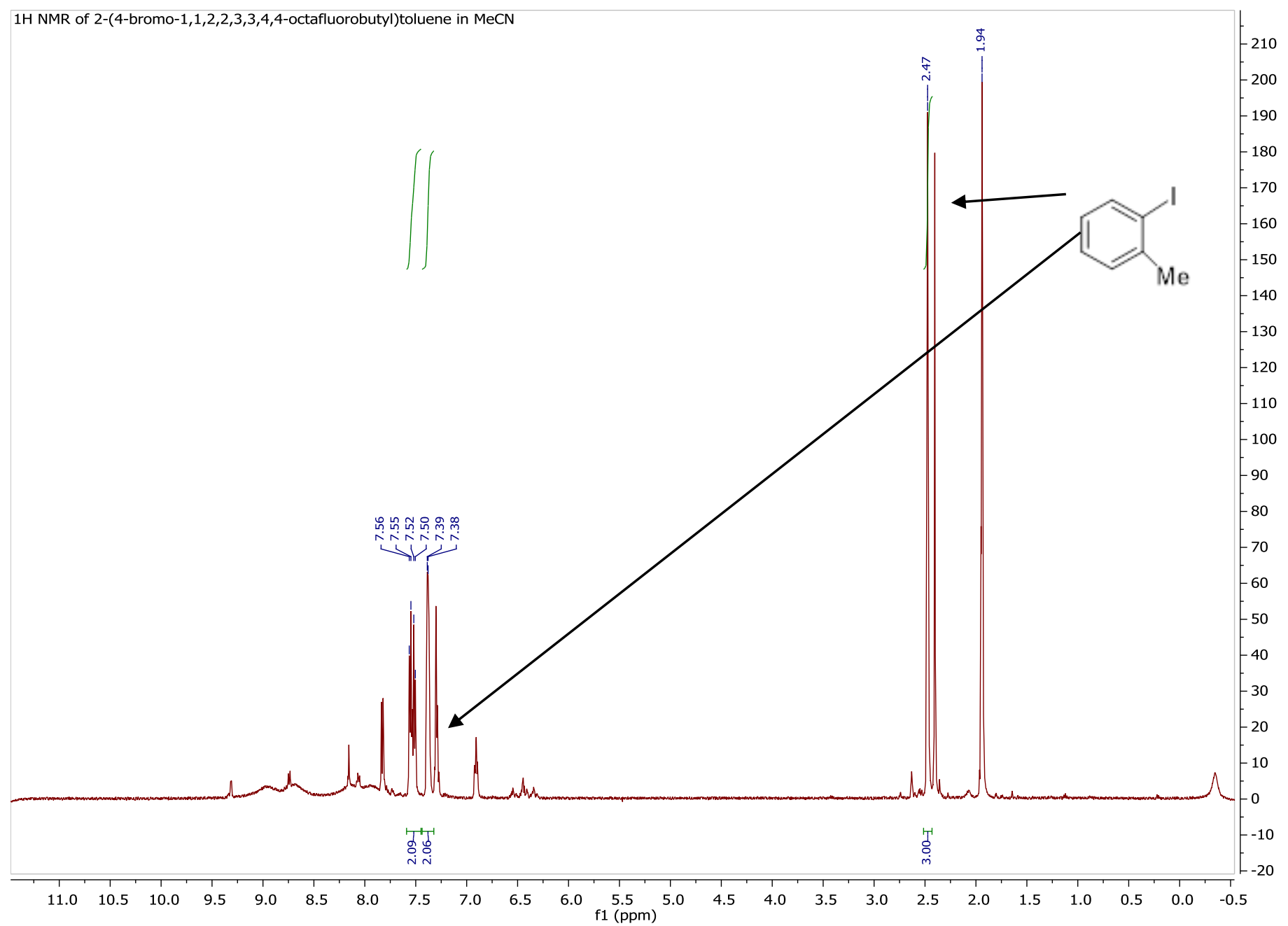




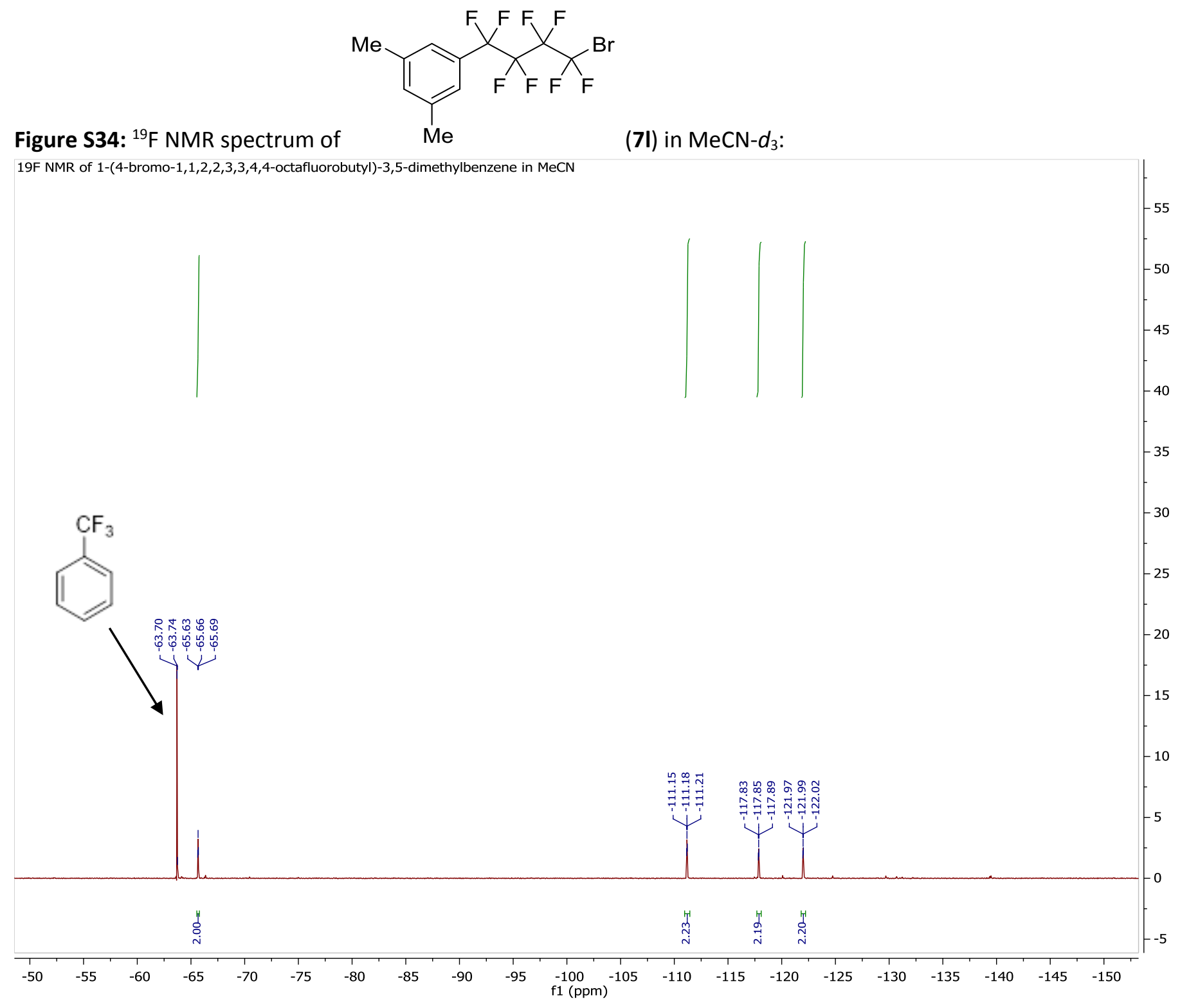




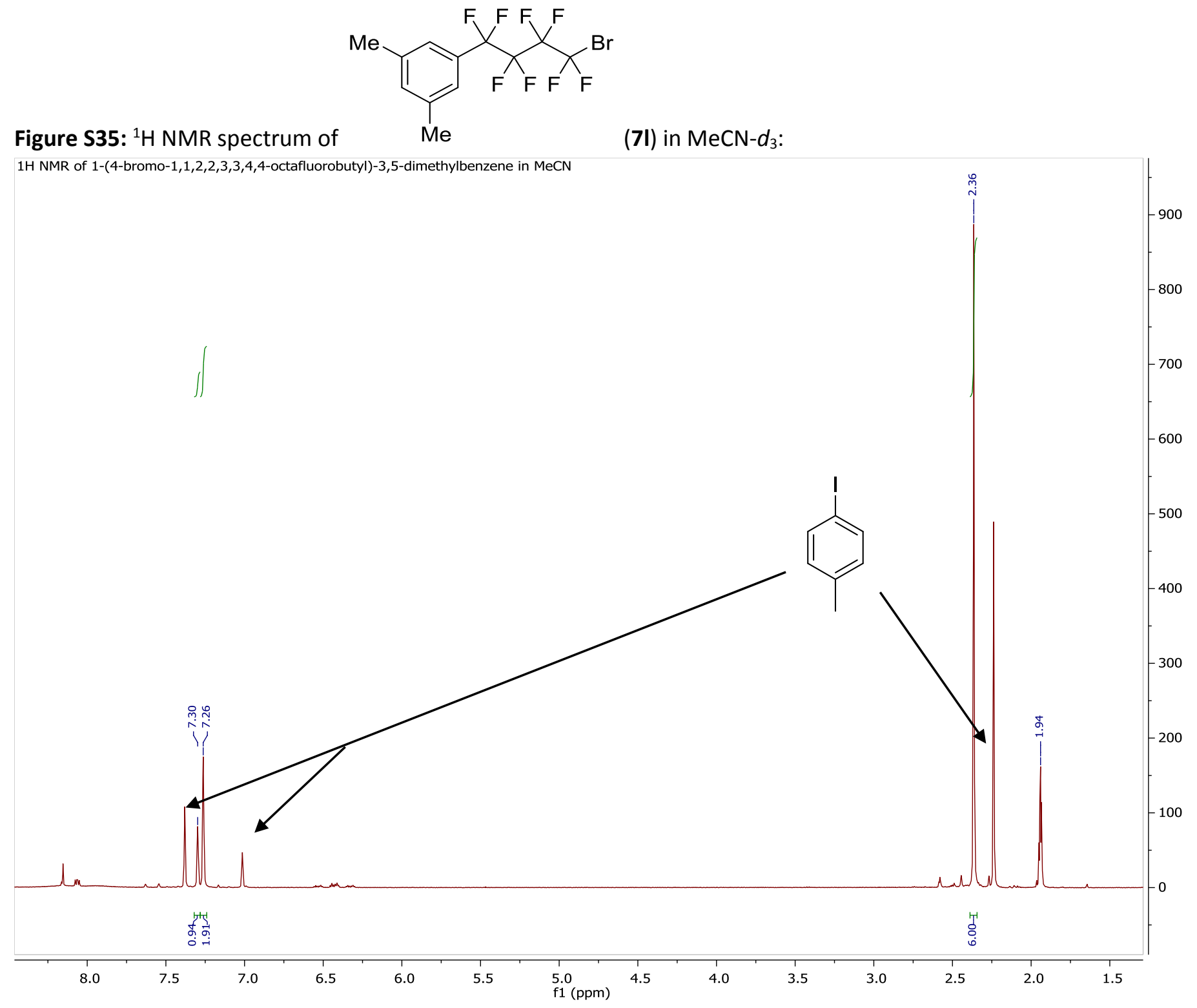


Figure S36: ${ }^{19} \mathrm{~F}$ NMR spectrum of

19F NMR of 1-(4-bromo-1,1,2,2,3,3,4,4-octafluorobutyl)-4-tertbutylbenzene in MeCN
$(7 \mathrm{~m})$ in $\mathrm{MeCN}-d_{3}:$

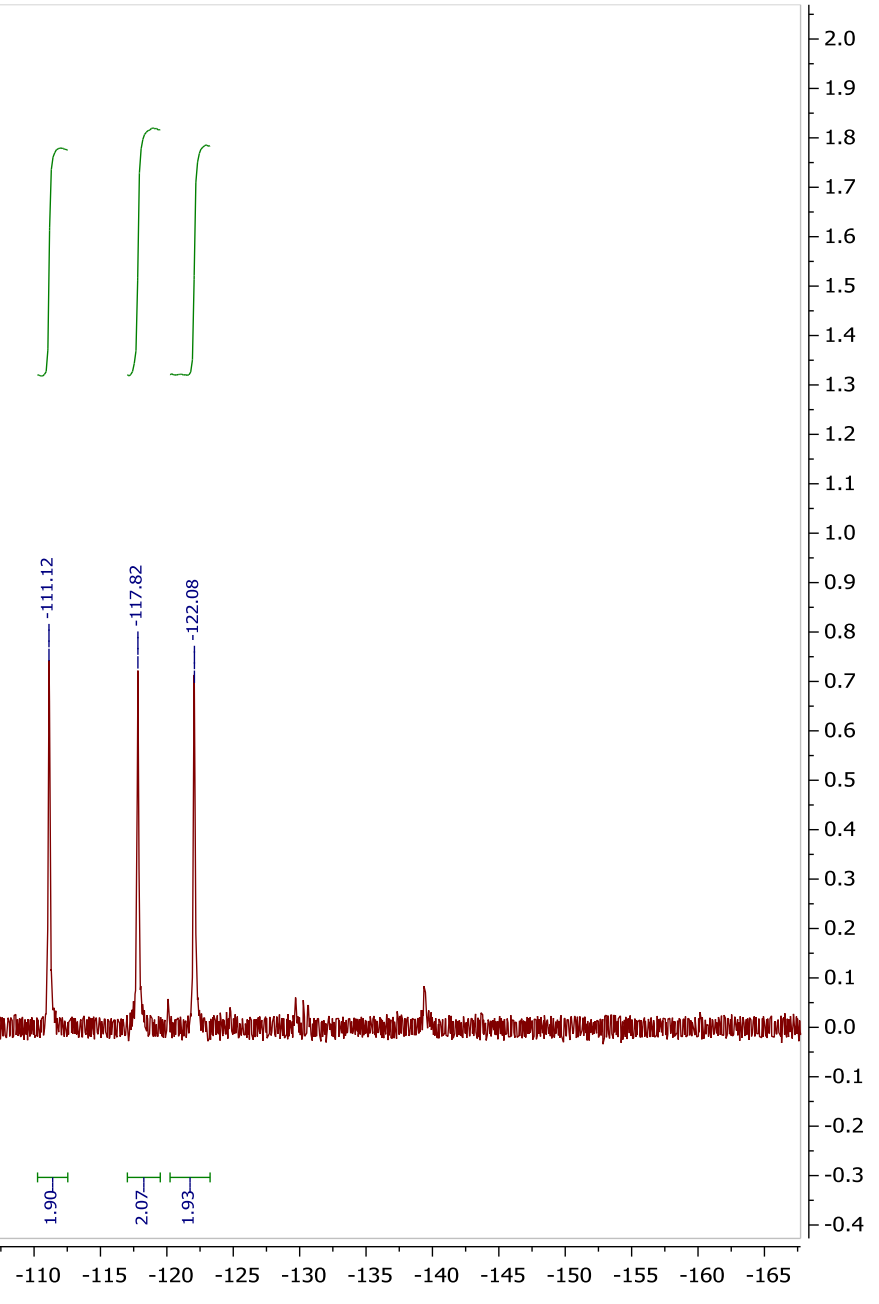


Figure 537: ${ }^{1} \mathrm{H}$ NMR spectrum of

$1 \mathrm{H}$ NMR of 1-(4-bromo-1,1,2,2,3,3,4,4-octafluorobutyl)-4-tertbutylbenzene in MeCN

$(7 \mathrm{~m})$ in $\mathrm{MeCN}-d_{3}$ :

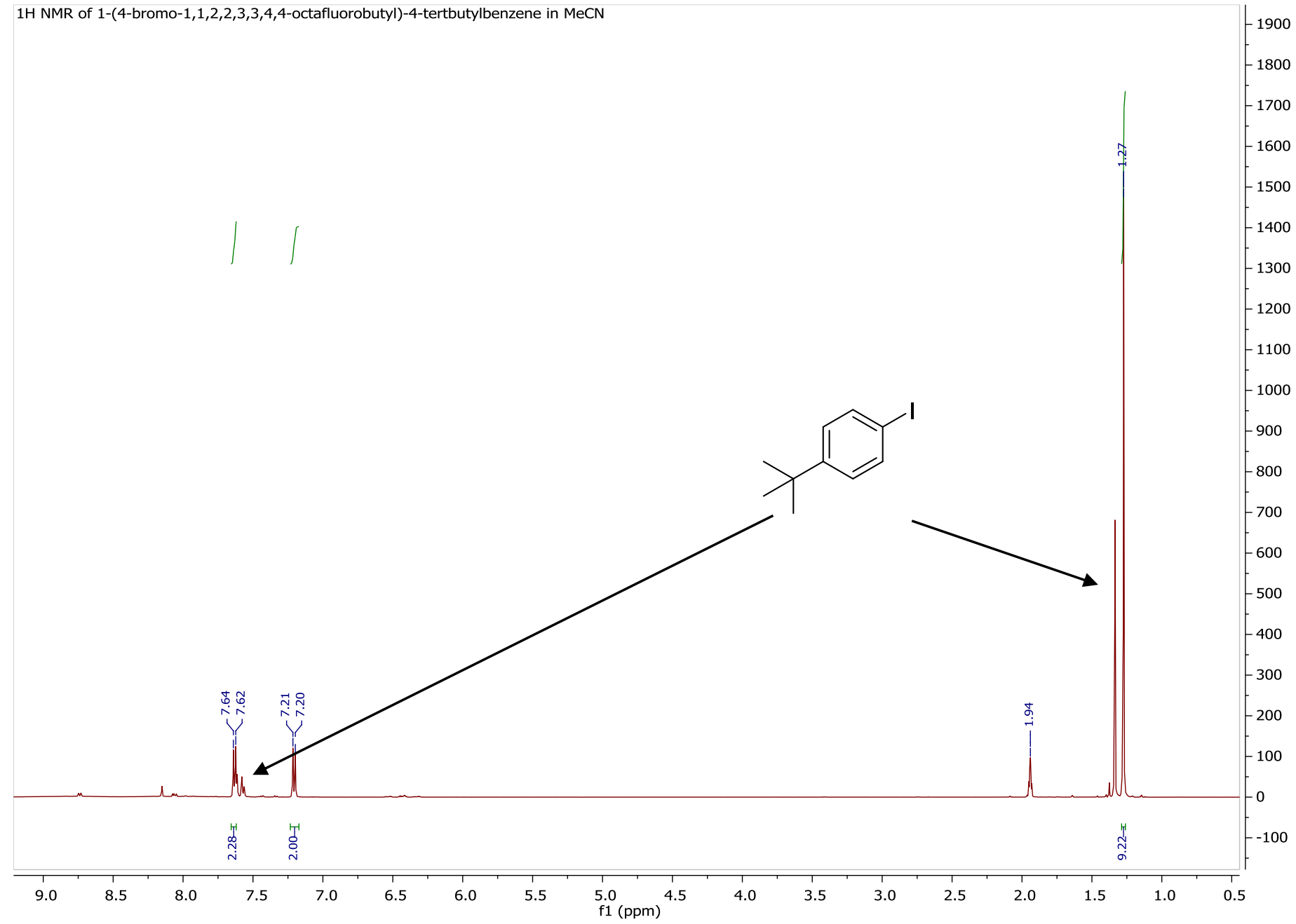




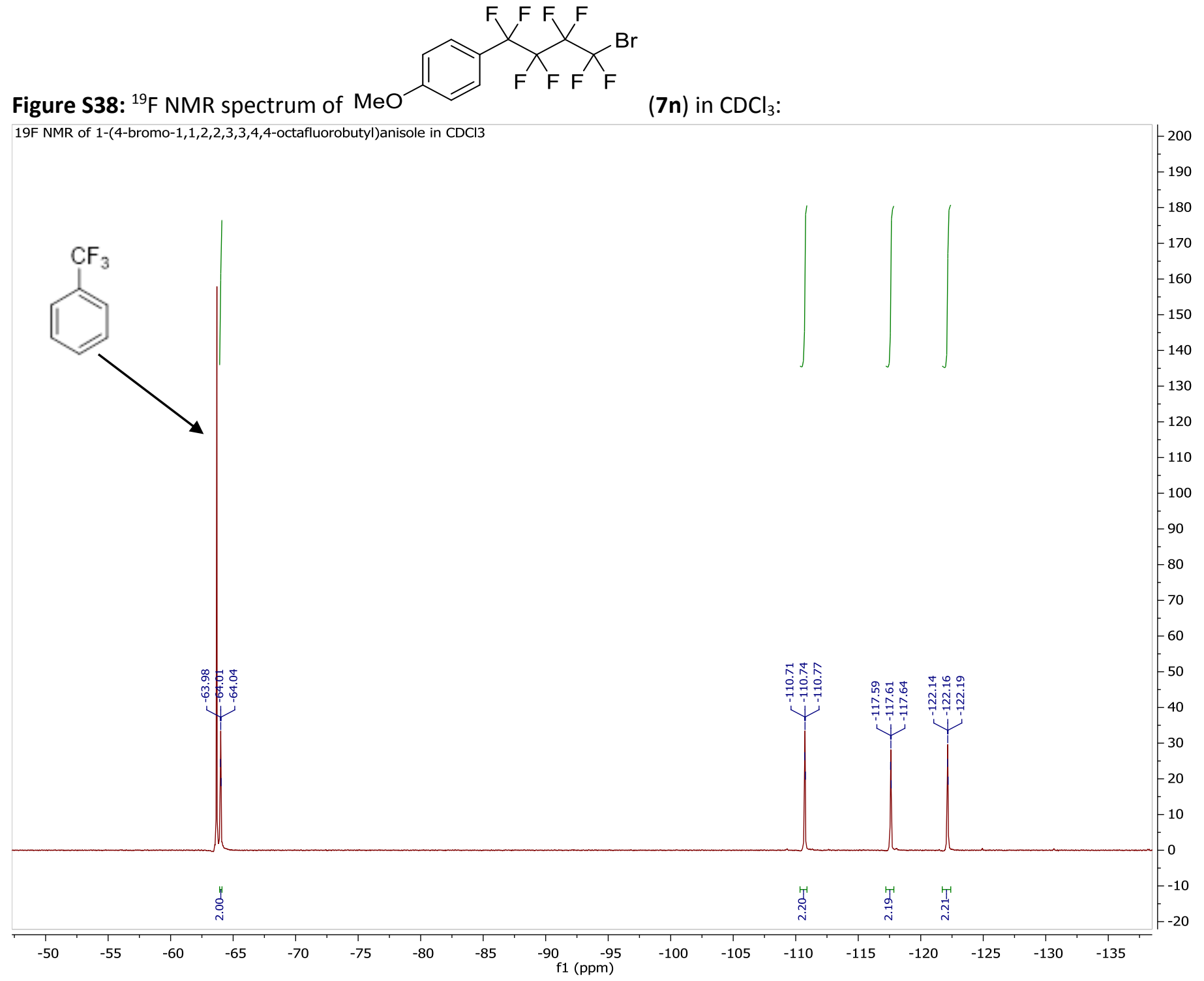




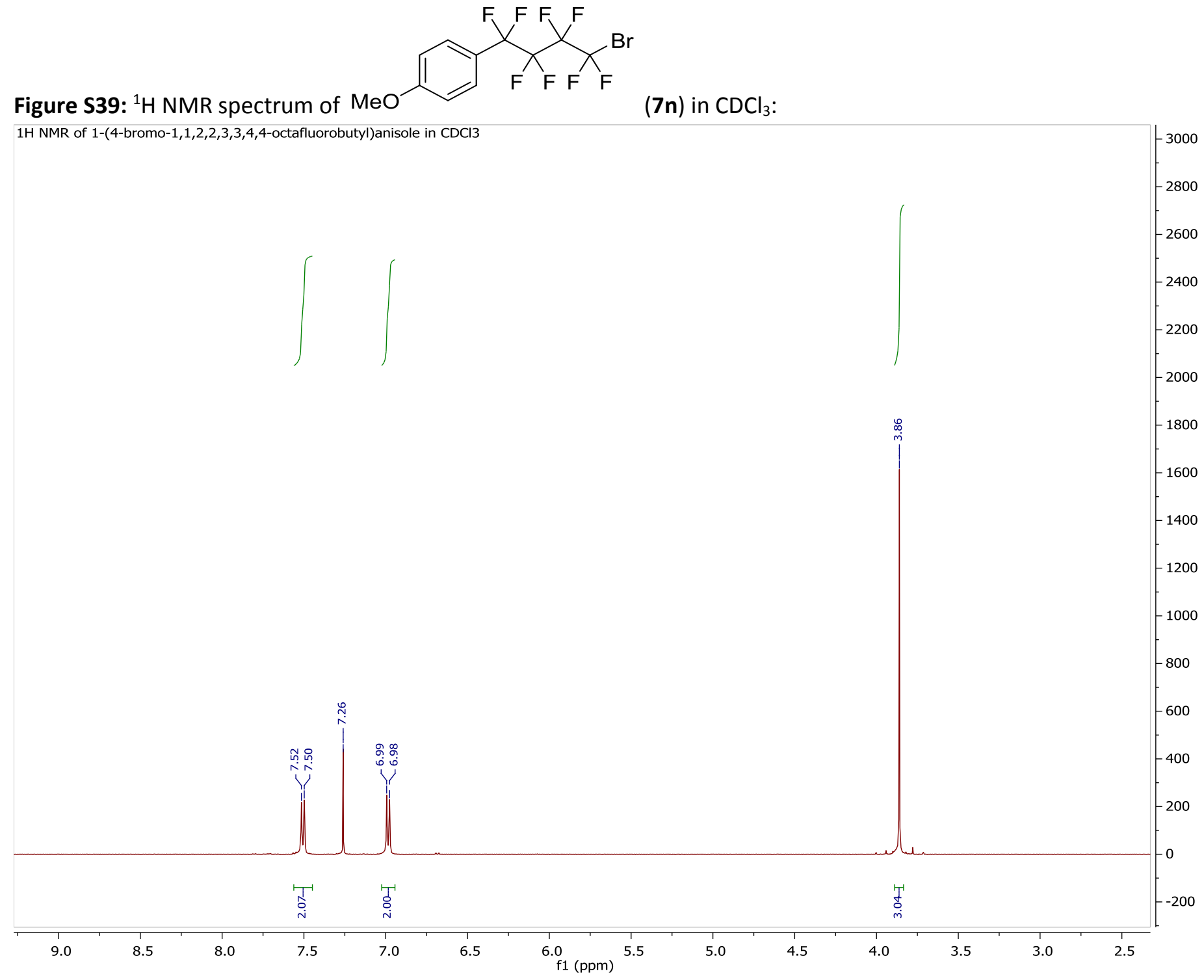


Figure S40: ${ }^{19} \mathrm{~F}$ NMR spectrum of $\mathrm{O}_{2} \mathrm{~N}^{2}$

$19 F$ NMR of 4-(4-bromo-1,1,2,2,3,3,4,4-octafluorobutyl)nitrobenzene in CDCl3

(7o) in $\mathrm{CDCl}_{3}$ :

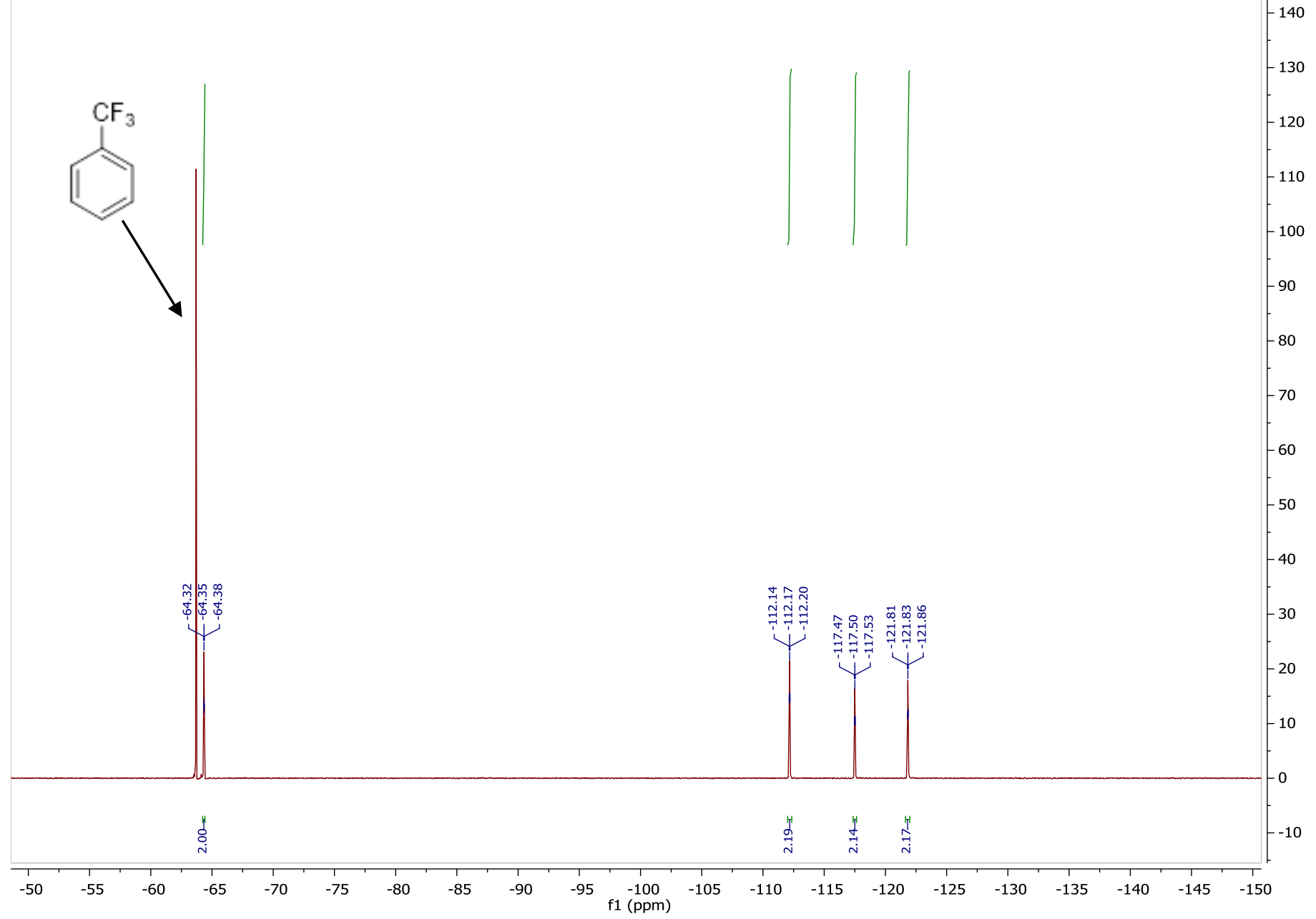


Figure S41: ${ }^{1} \mathrm{H}$ NMR spectrum of $\mathrm{O}_{2} \mathrm{~N}$

Figure S41: ${ }^{1} \mathrm{H}$ NMR spectrum of $\mathrm{O}_{2} \mathrm{~N}$ (7o) in $\mathrm{CDCl}_{3}$ :

$1 \mathrm{H}$ NMR of 4-(4-bromo-1,1,2,2,3,3,4,4-octafluorobutyl)nitrobenzene in CDCl3

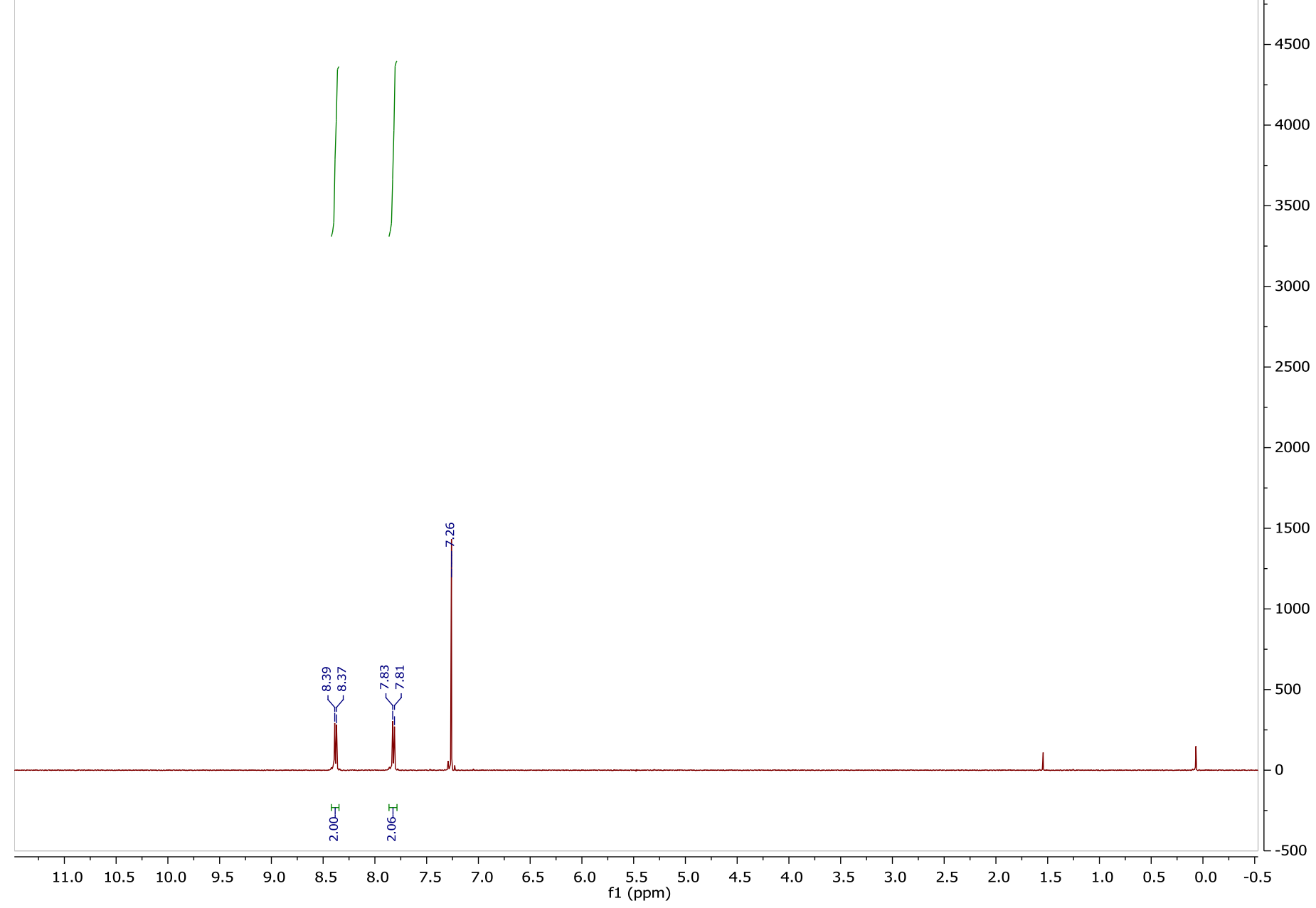




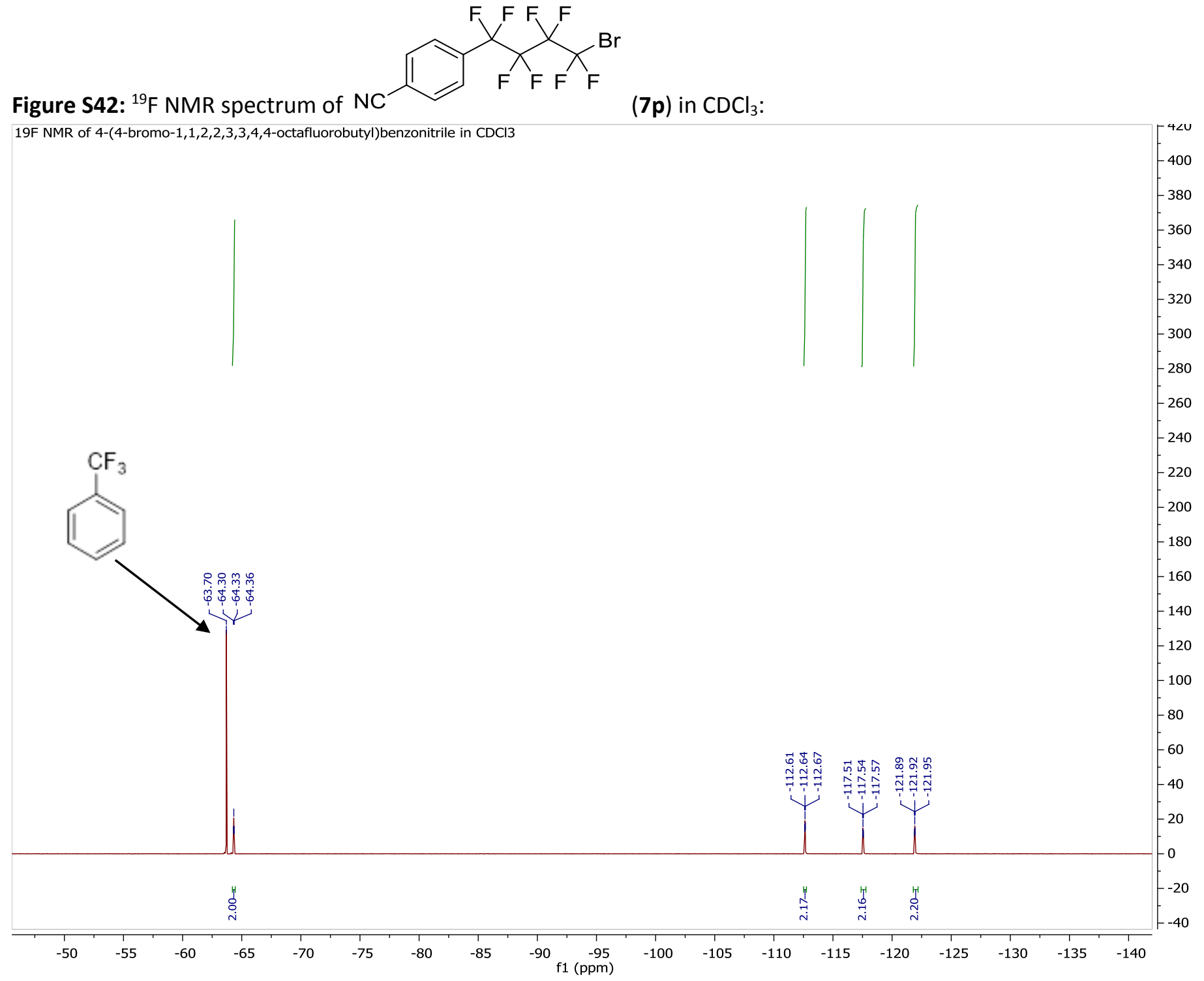




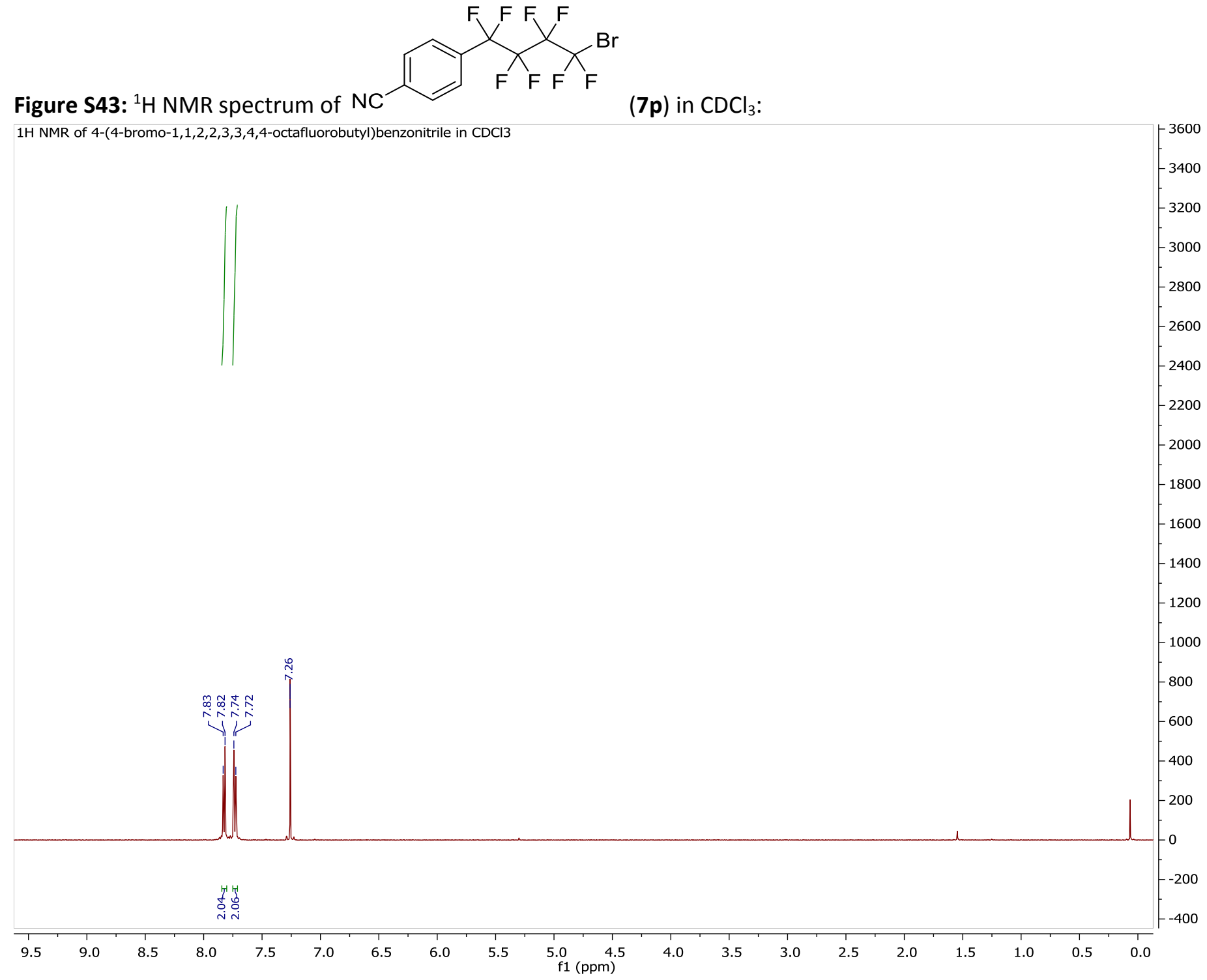




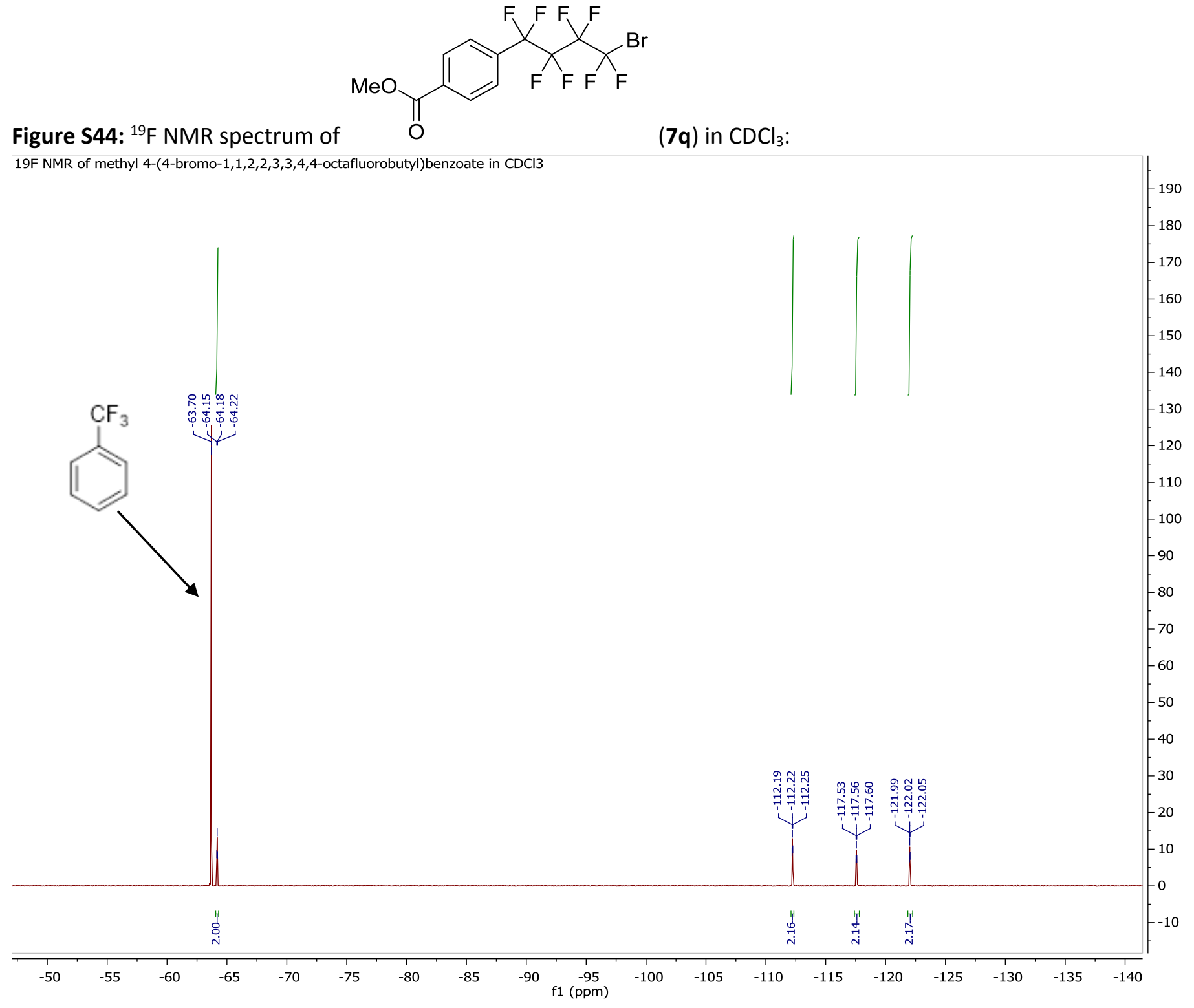




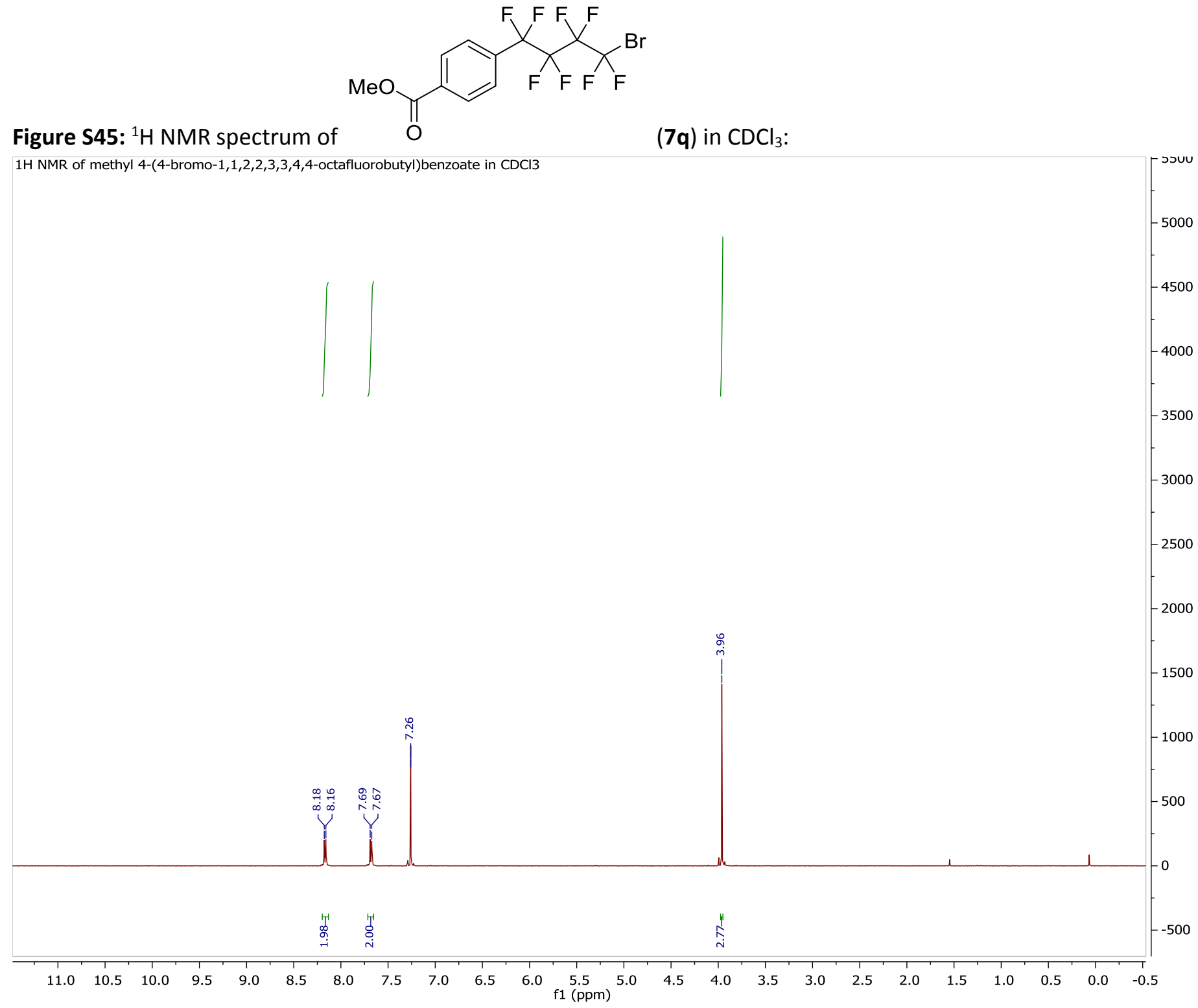




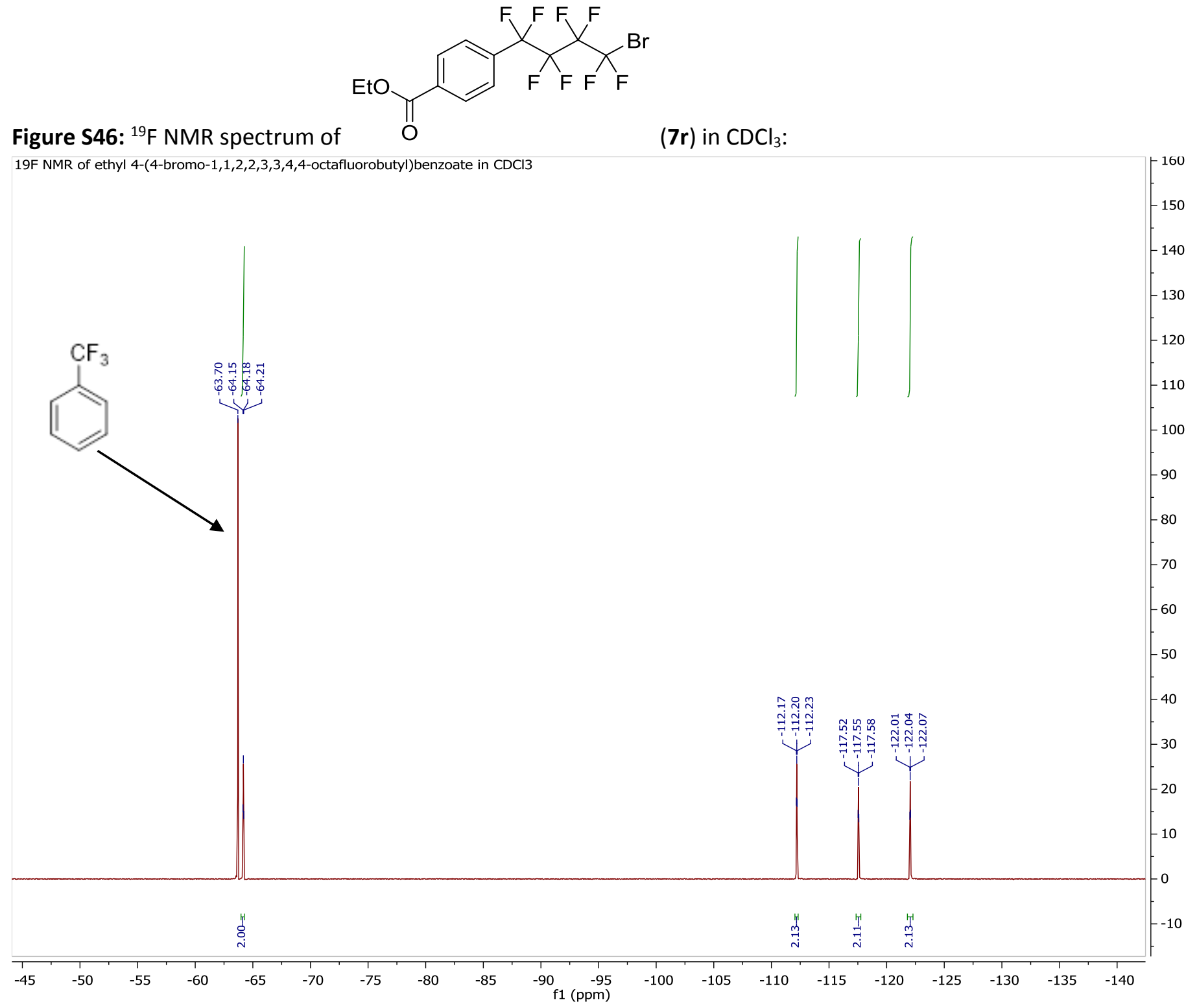


Figure S47: ${ }^{1} \mathrm{H}$ NMR spectrum of

$1 \mathrm{H}$ NMR of ethyl 4-(4-bromo-1,1,2,2,3,3,4,4-octafluorobutyl)benzoate in CDCl3

(7r) in $\mathrm{CDCl}_{3}$ :

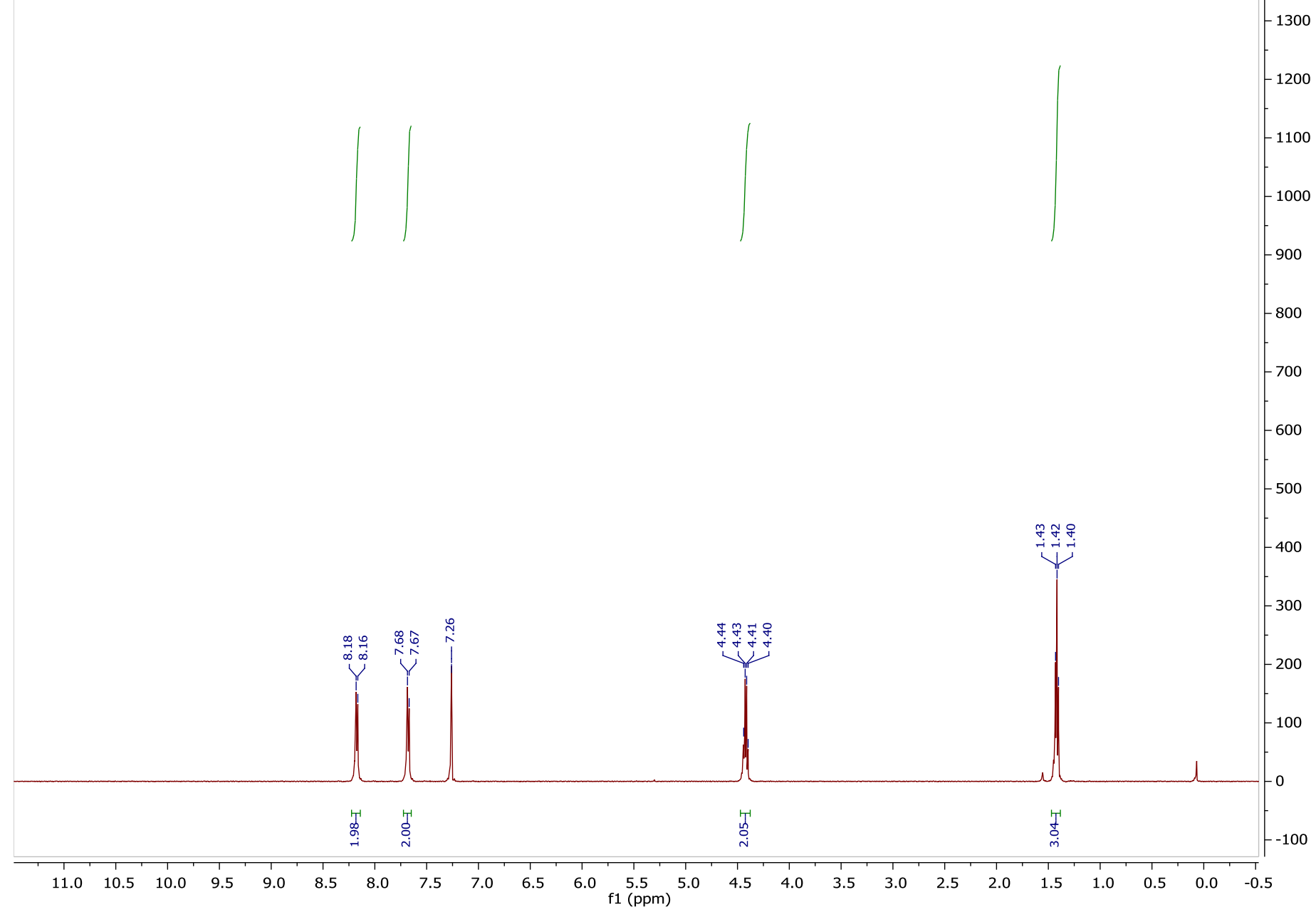




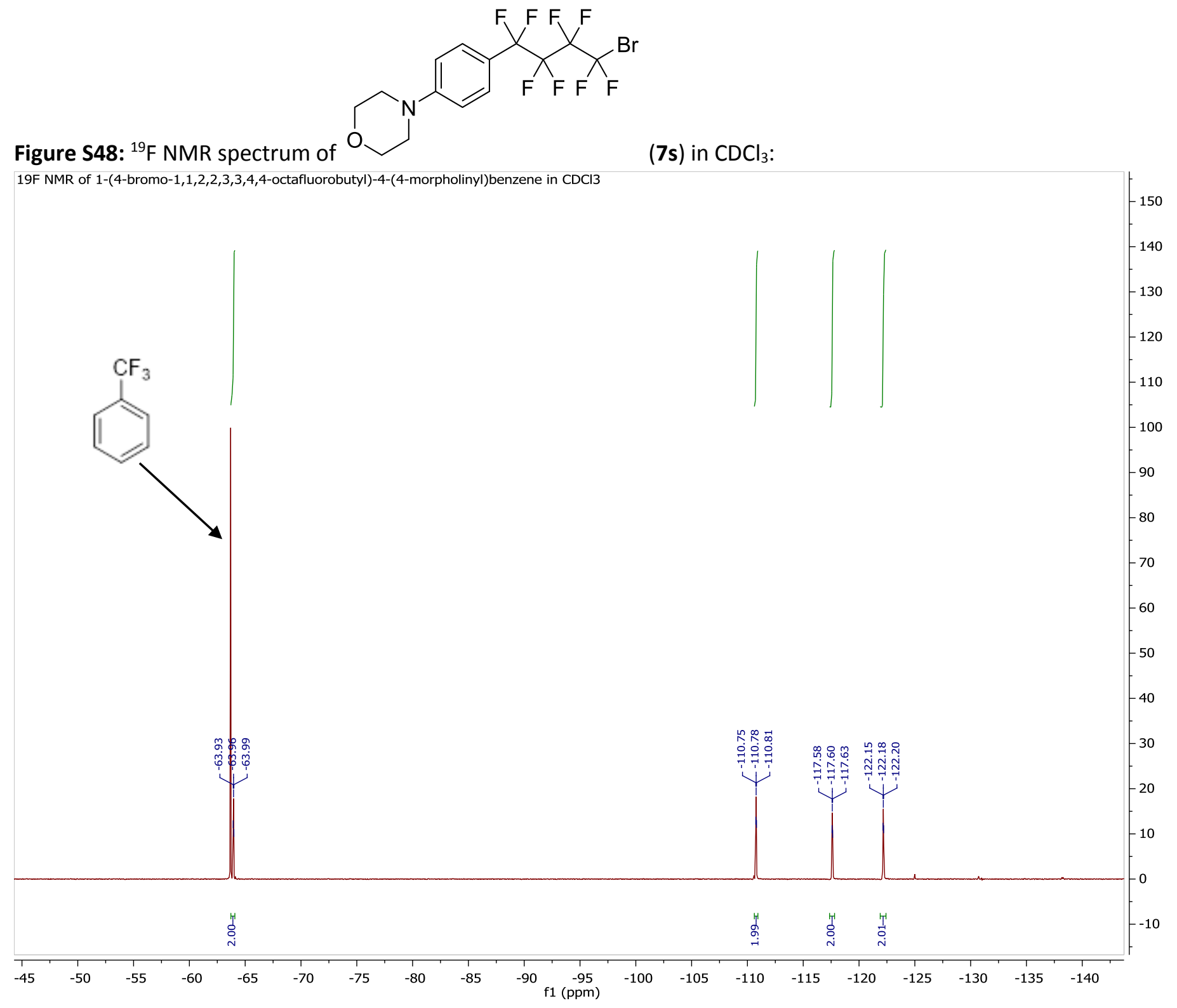


Figure S49: ${ }^{1} \mathrm{H}$ NMR spectrum of

$1 \mathrm{H}$ NMR of 1-(4-bromo-1,1,2,2,3,3,4,4-octafluorobutyl)-4-(4-morpholinyl)benzene in CDCl3

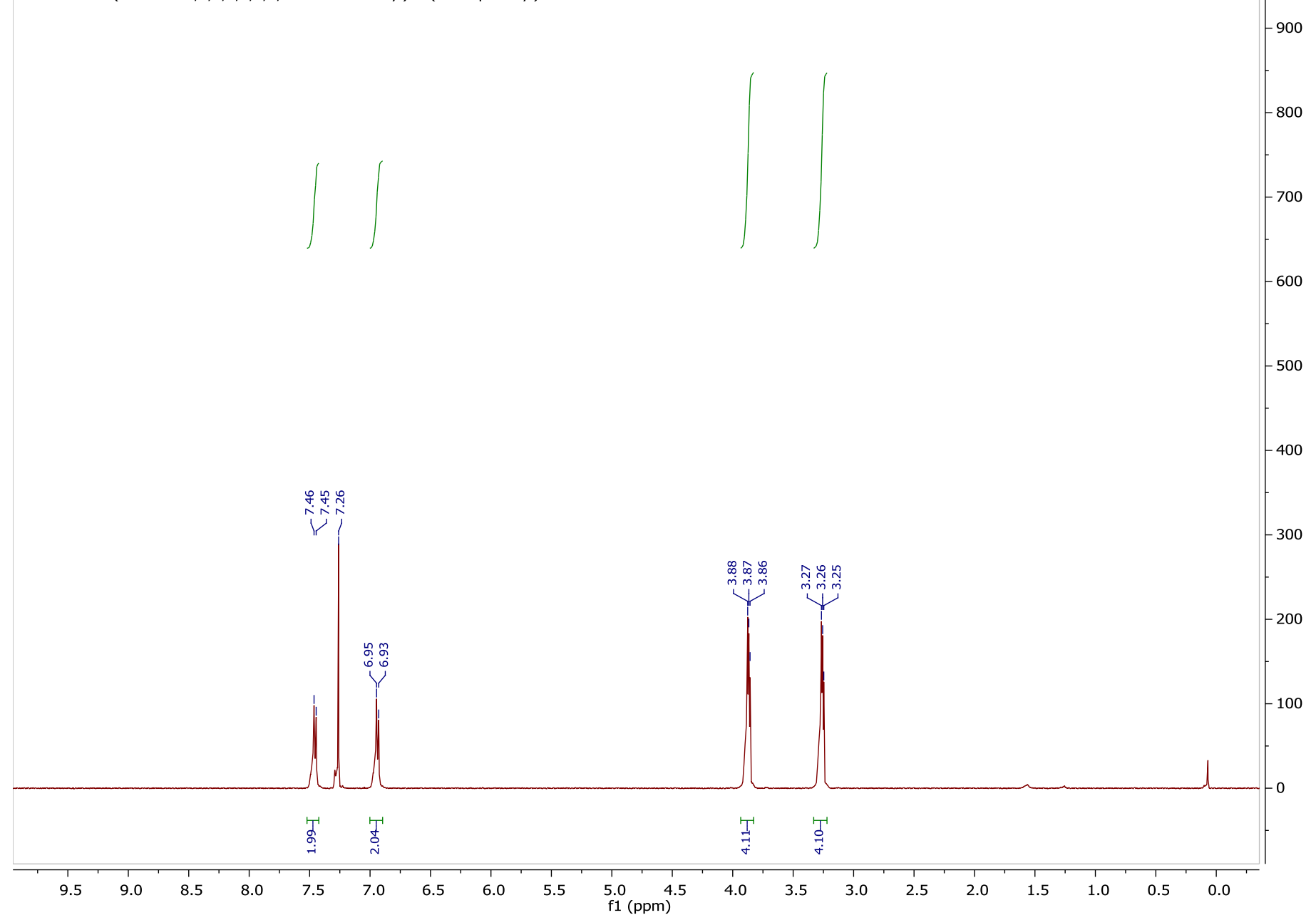

(7s) in $\mathrm{CDCl}_{3}$ : $1(\mathrm{ppm})$ 


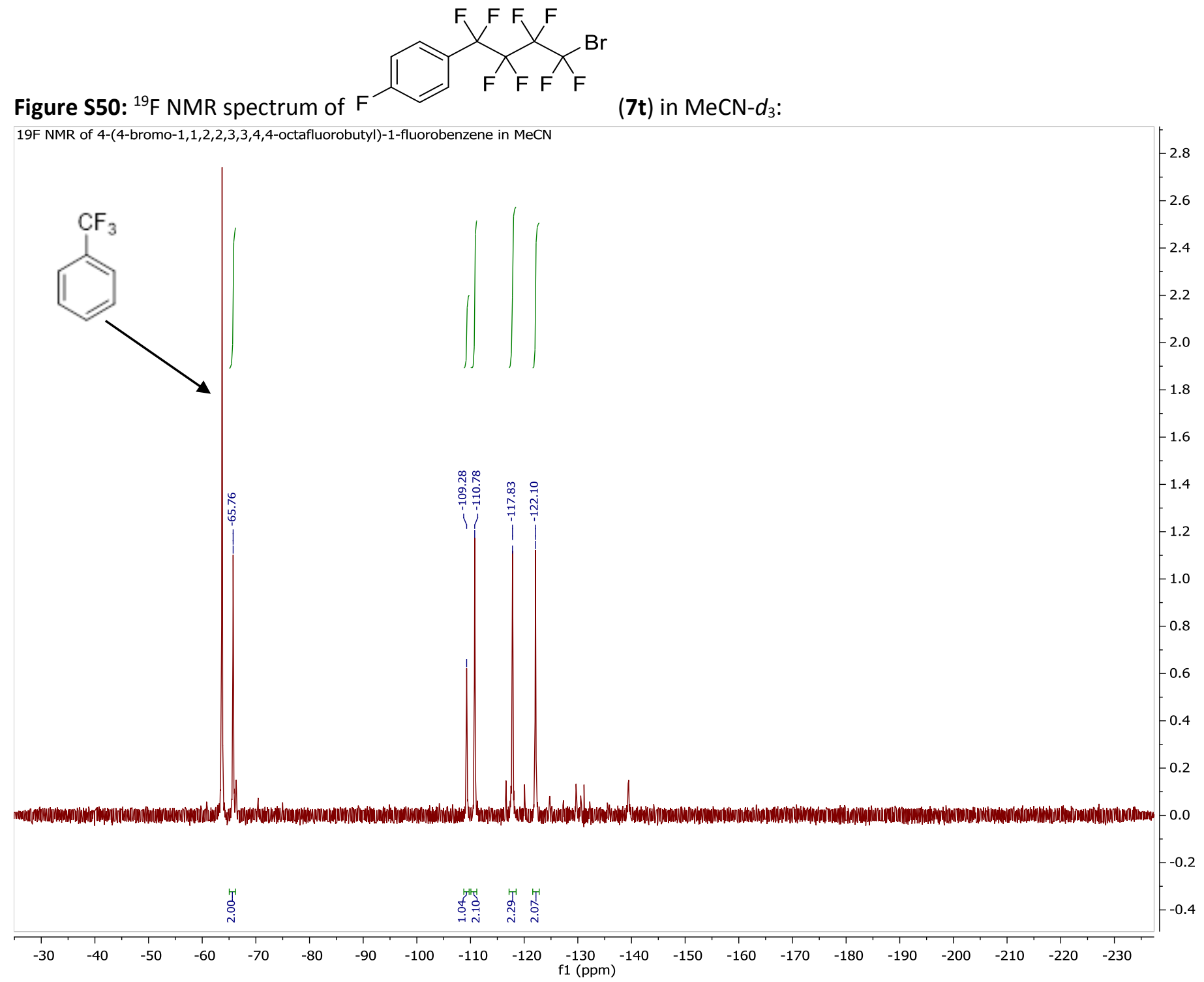




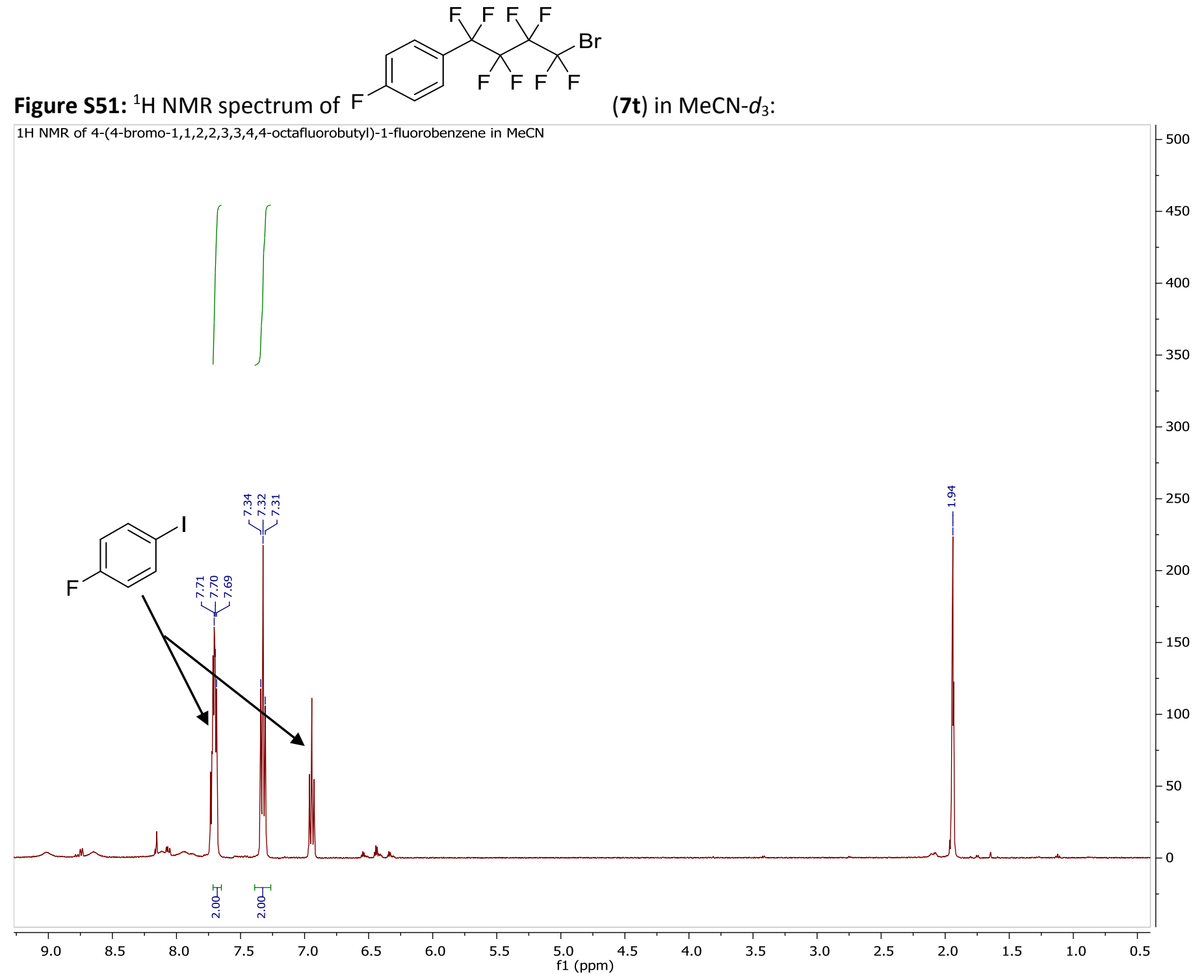




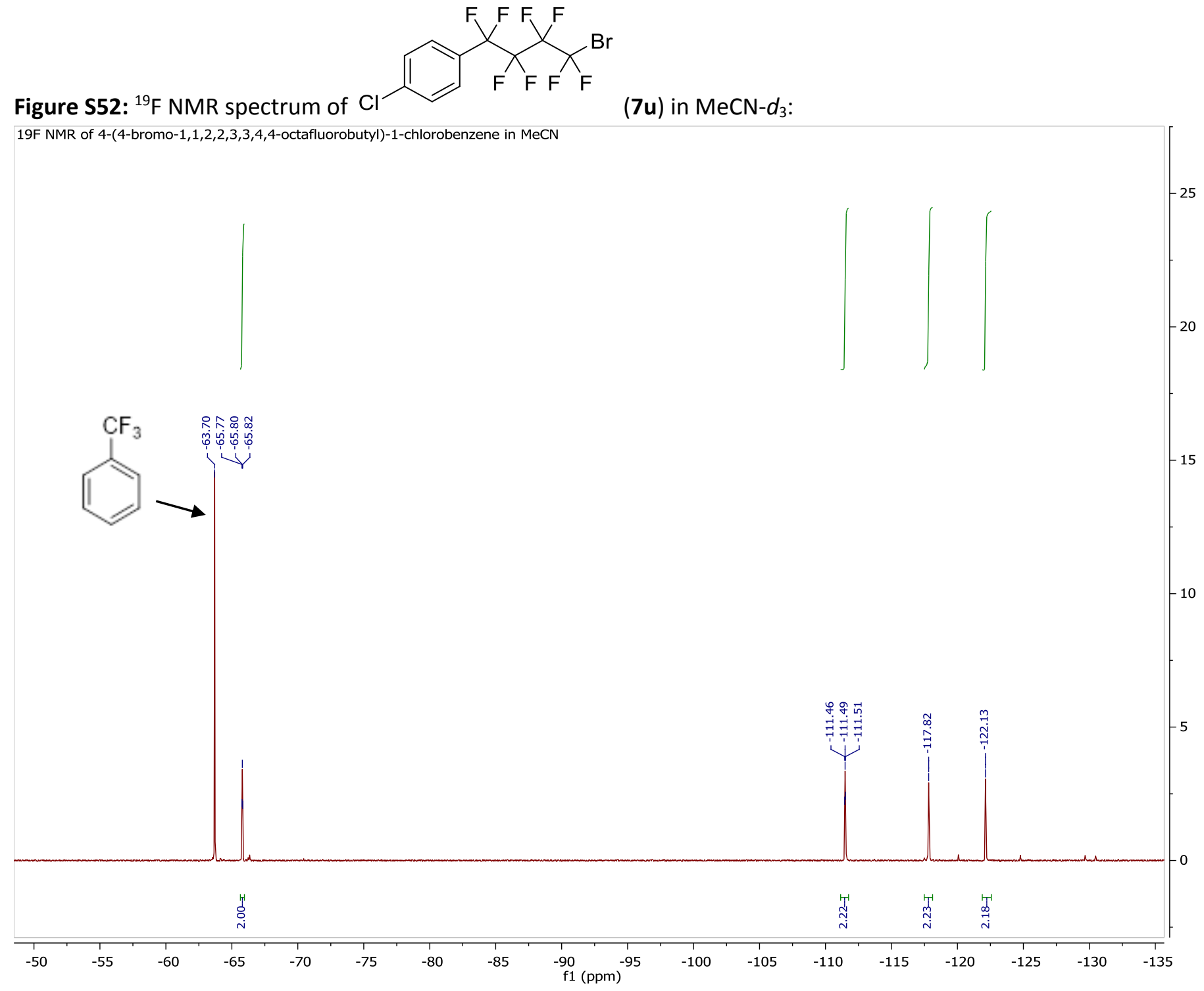




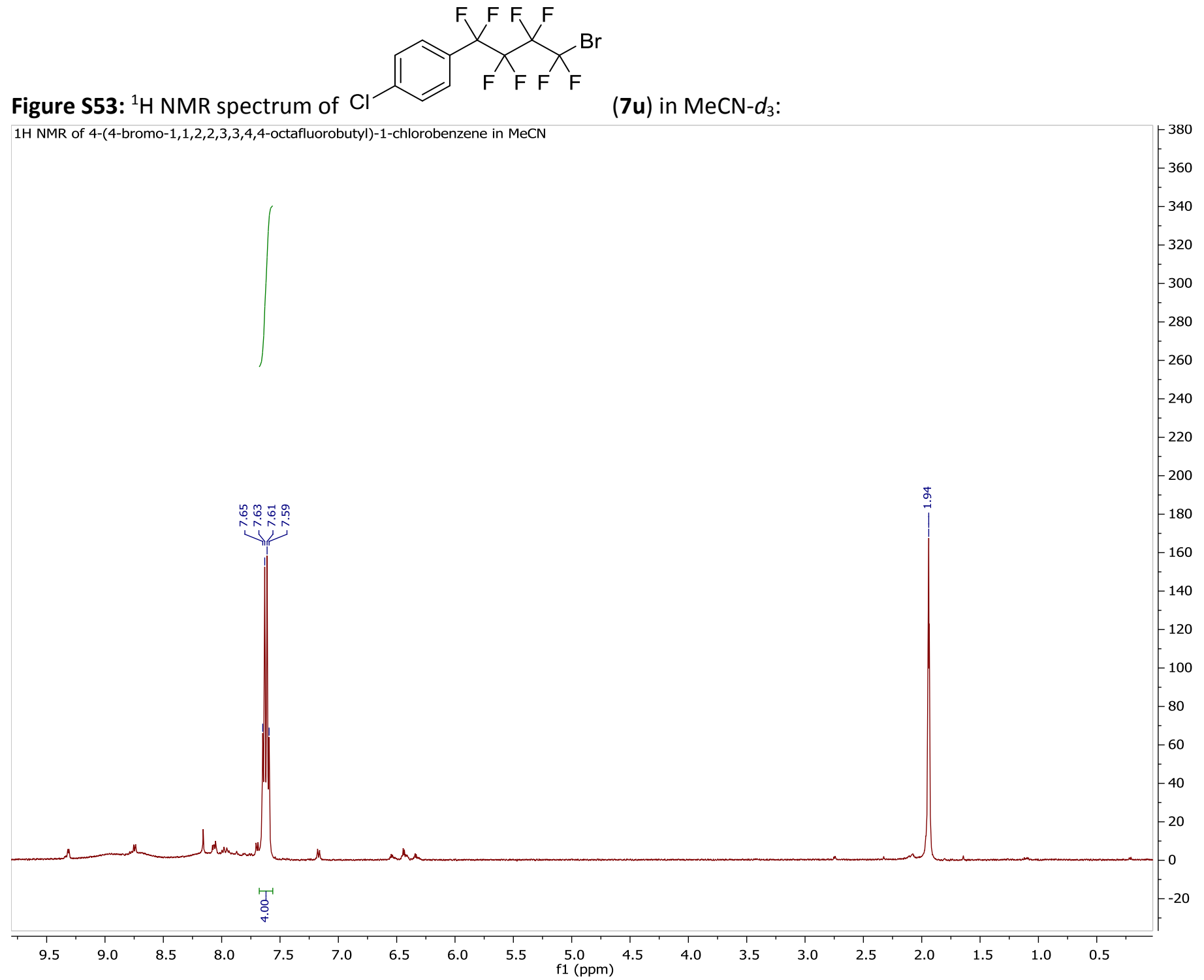




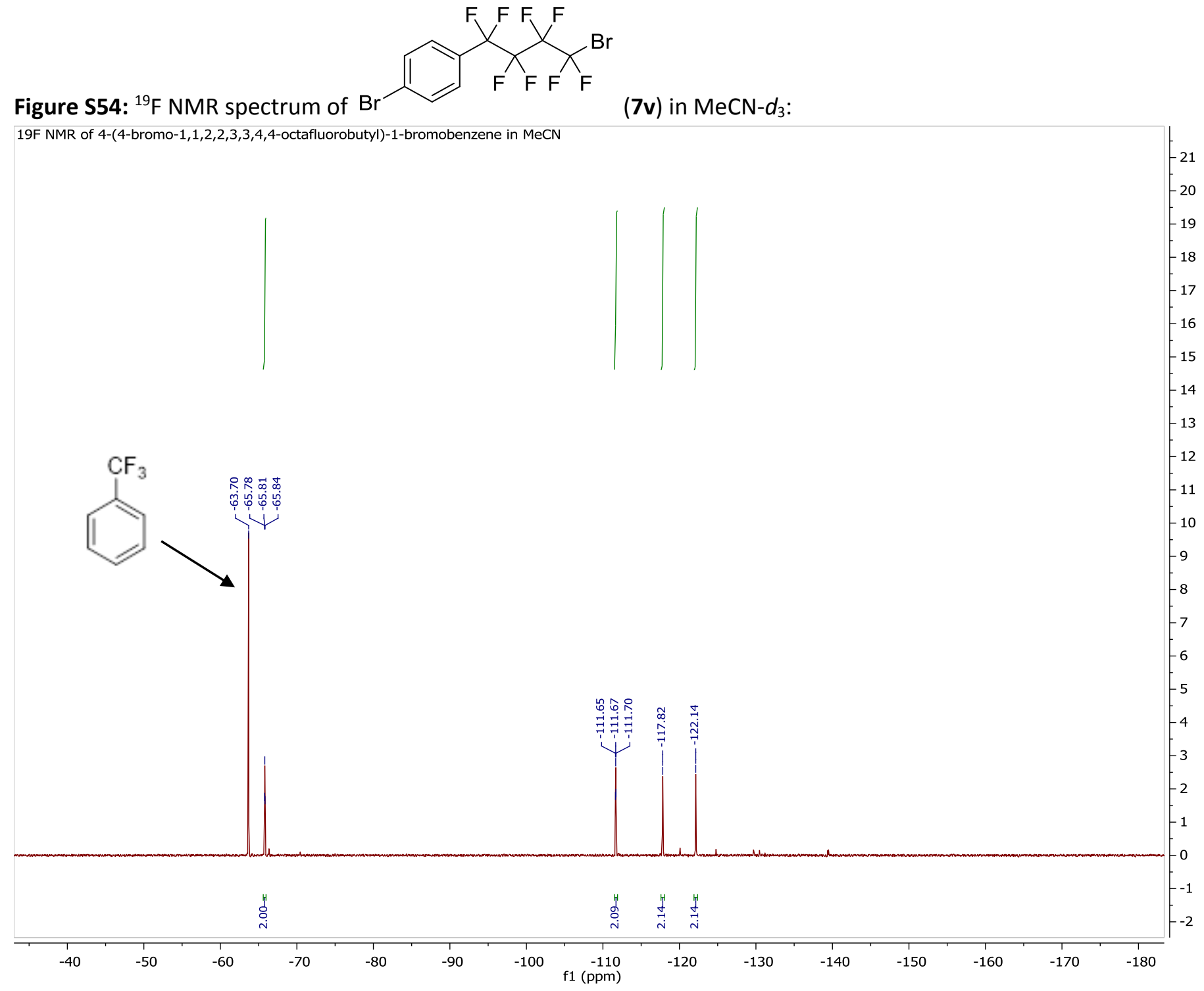




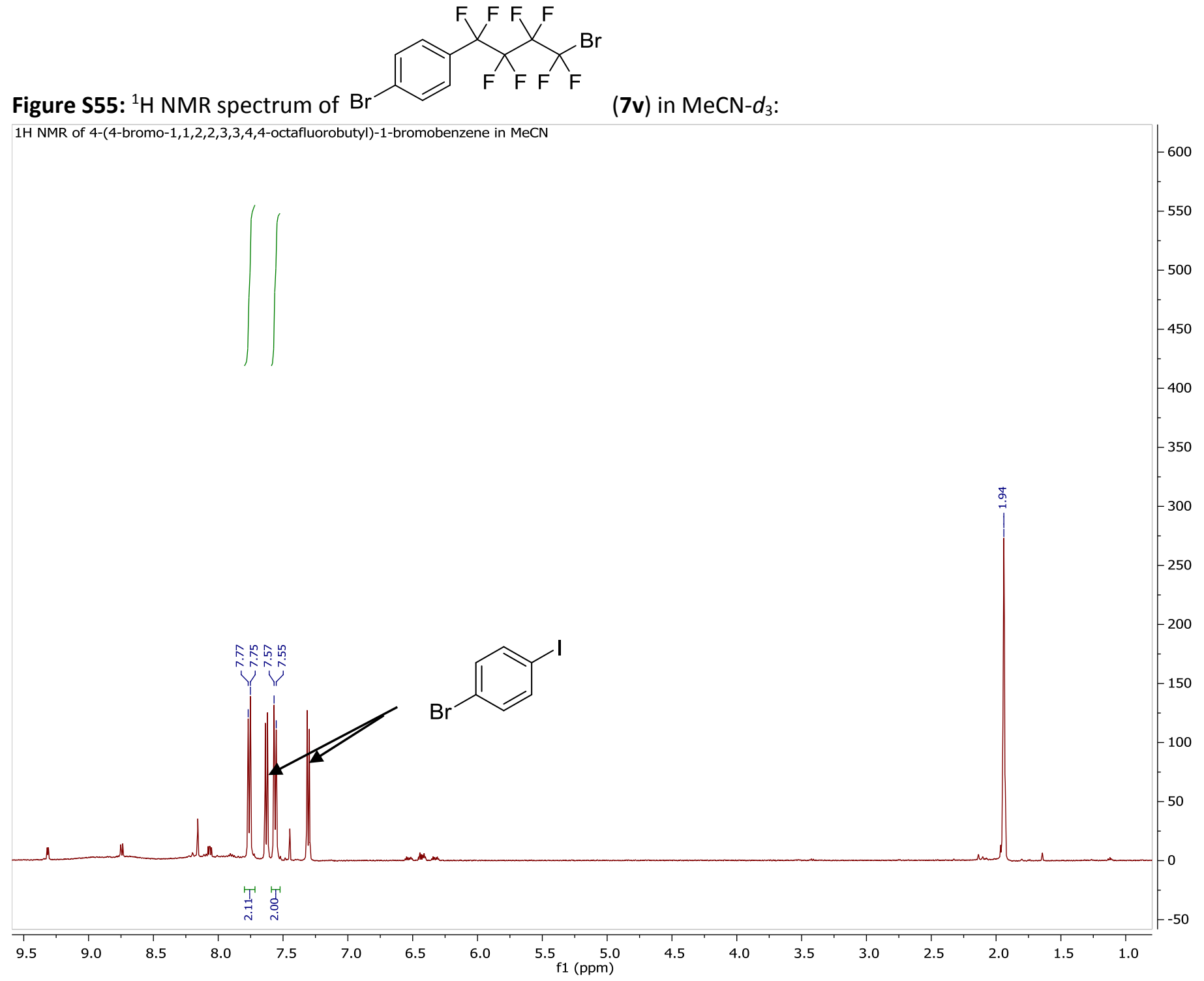




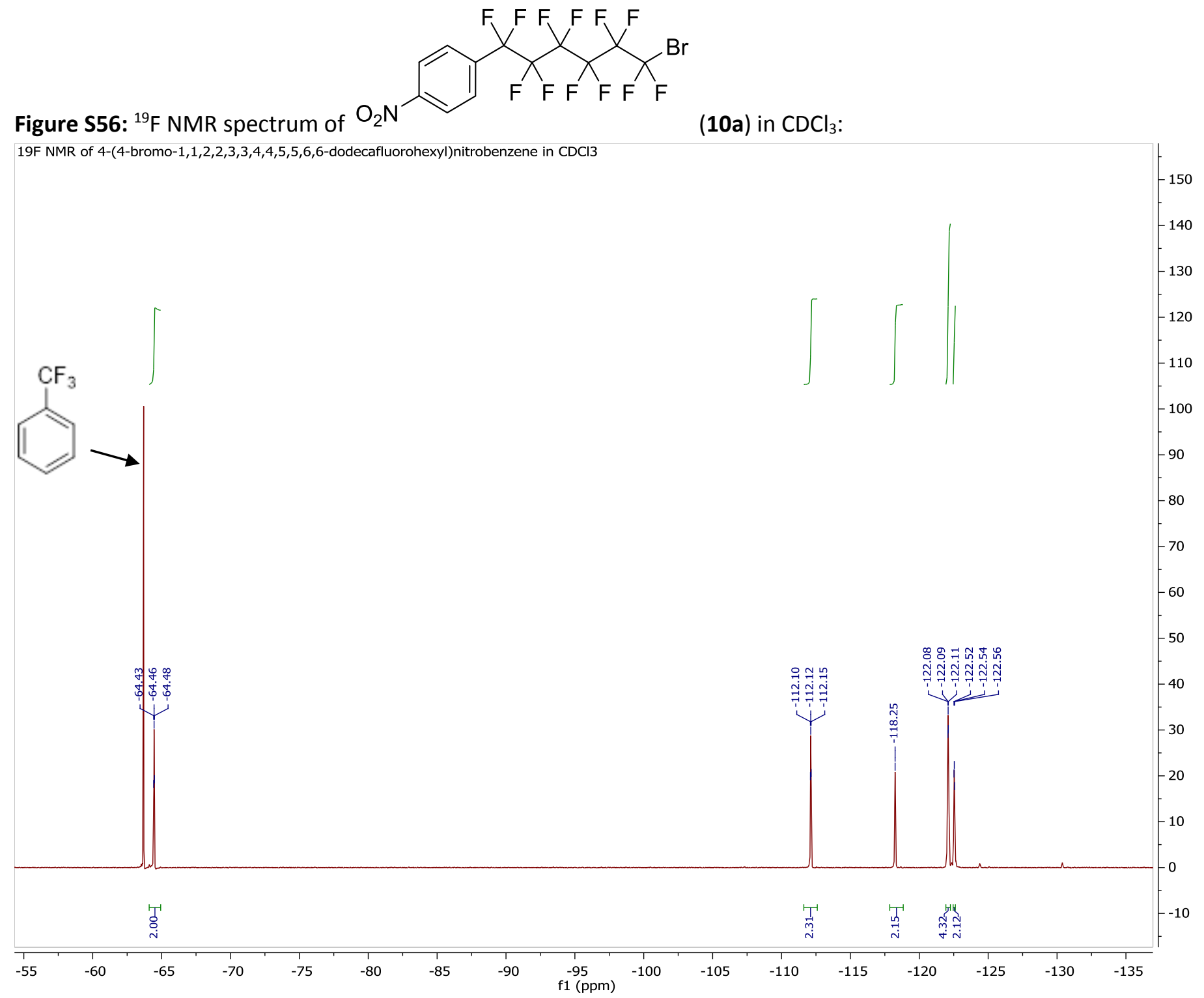




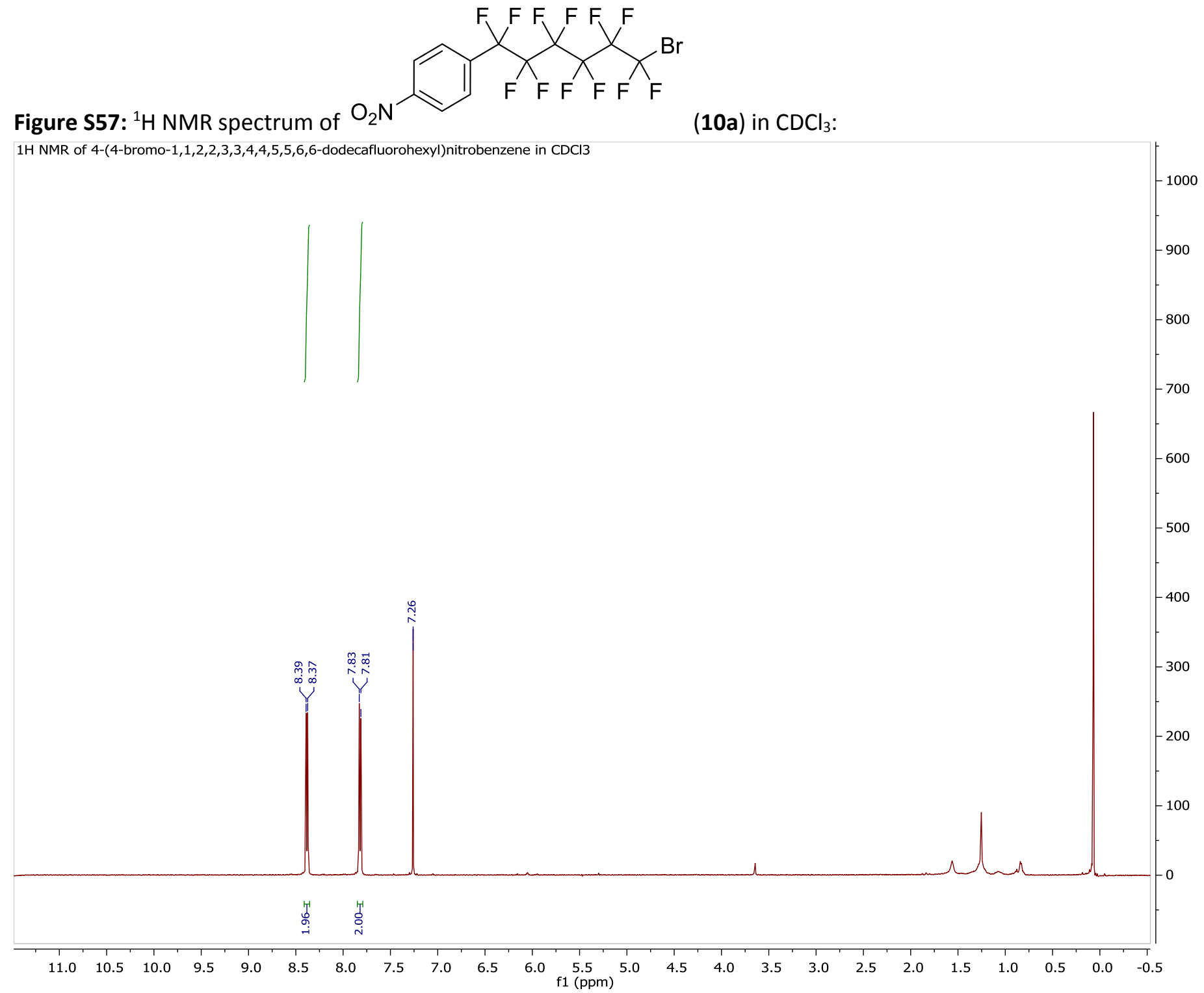




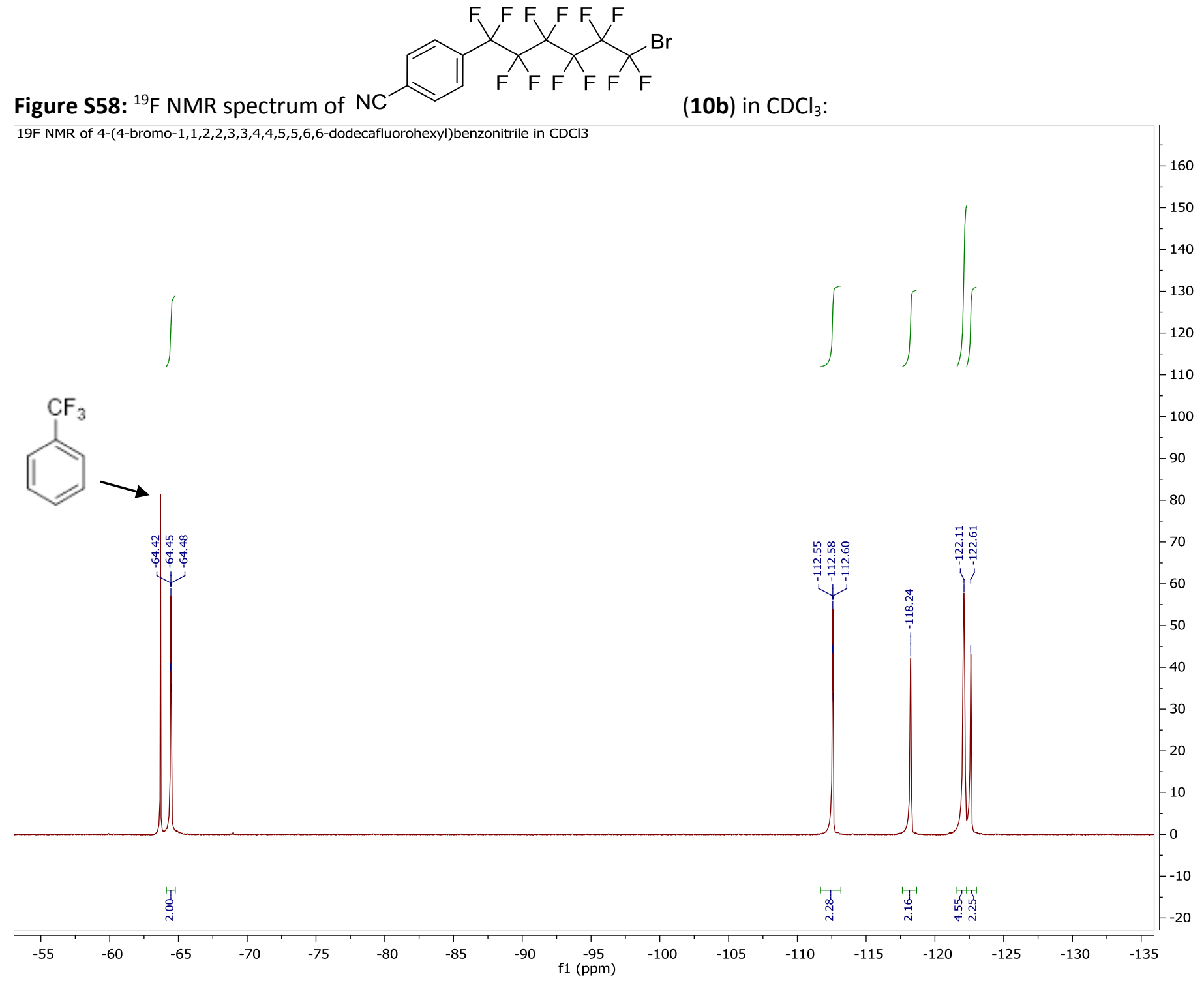




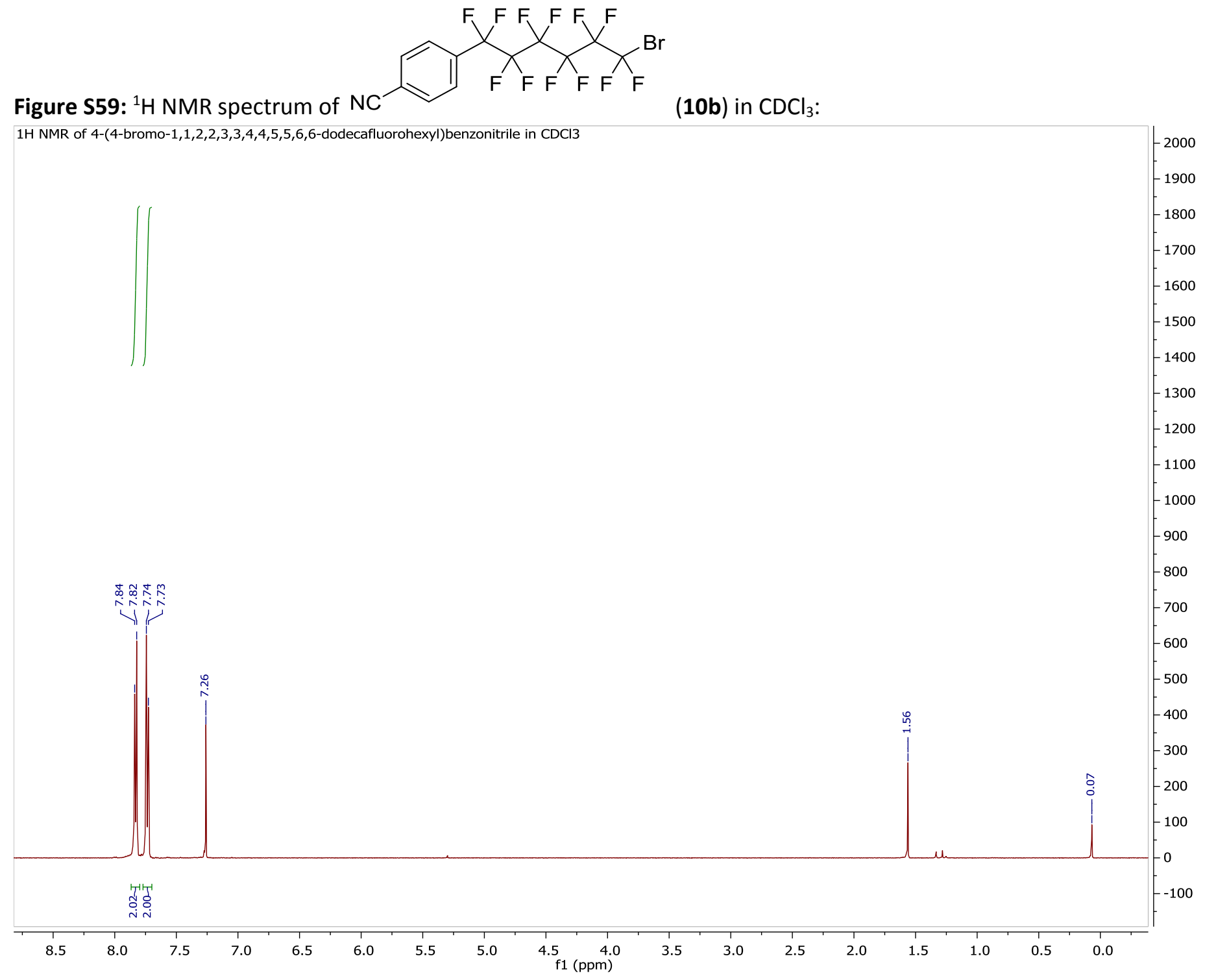


Figure S60: ${ }^{19} \mathrm{~F} N \mathrm{NMR}$ spectrum of

$19 F$ NMR of methyl 4-(4-bromo-1,1,2,2,3,3,4,4,5,5,6,6-dodecafluorohexyl)benzoate in CDCI3 (10c) in $\mathrm{CDCl}_{3}$ :

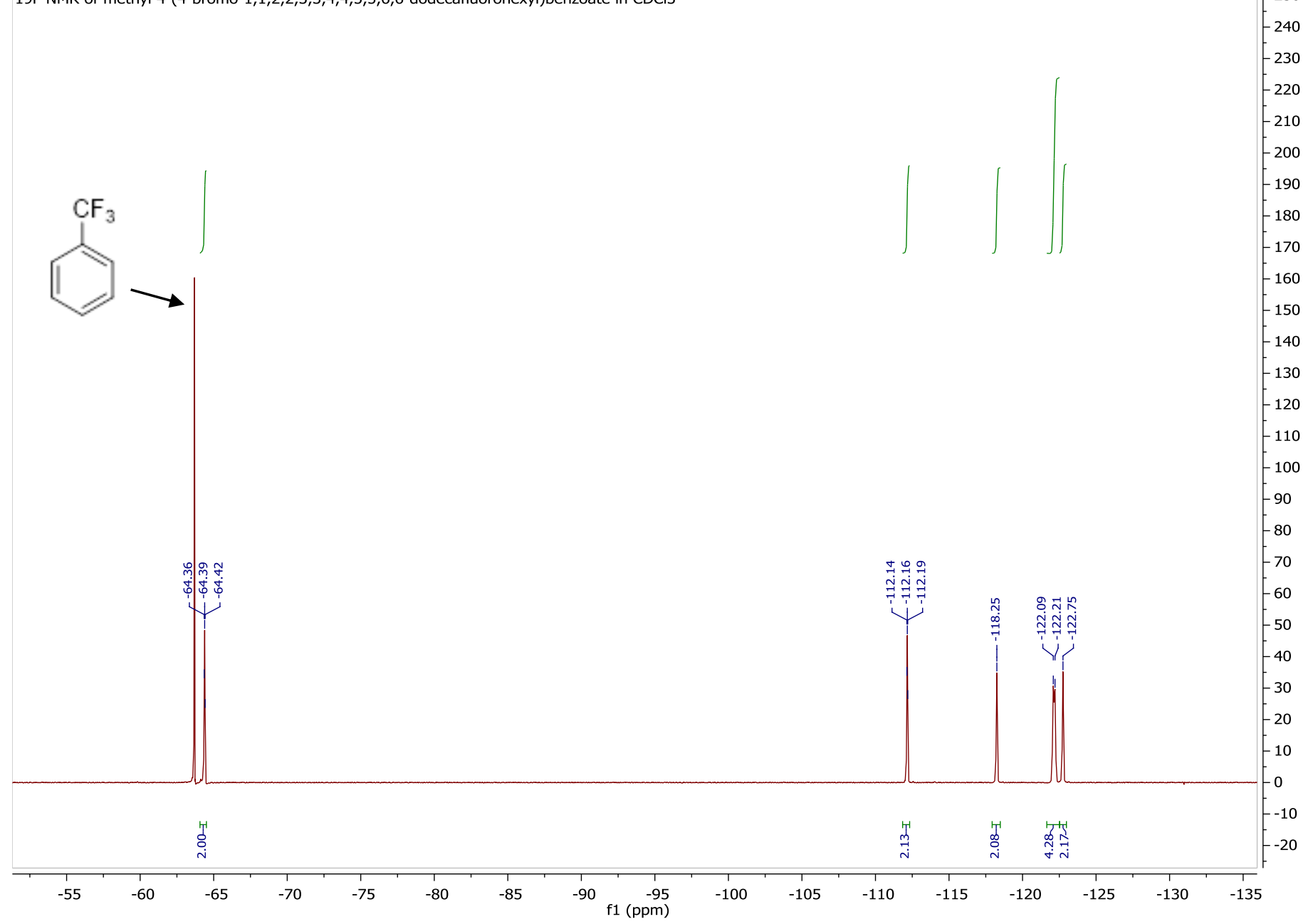




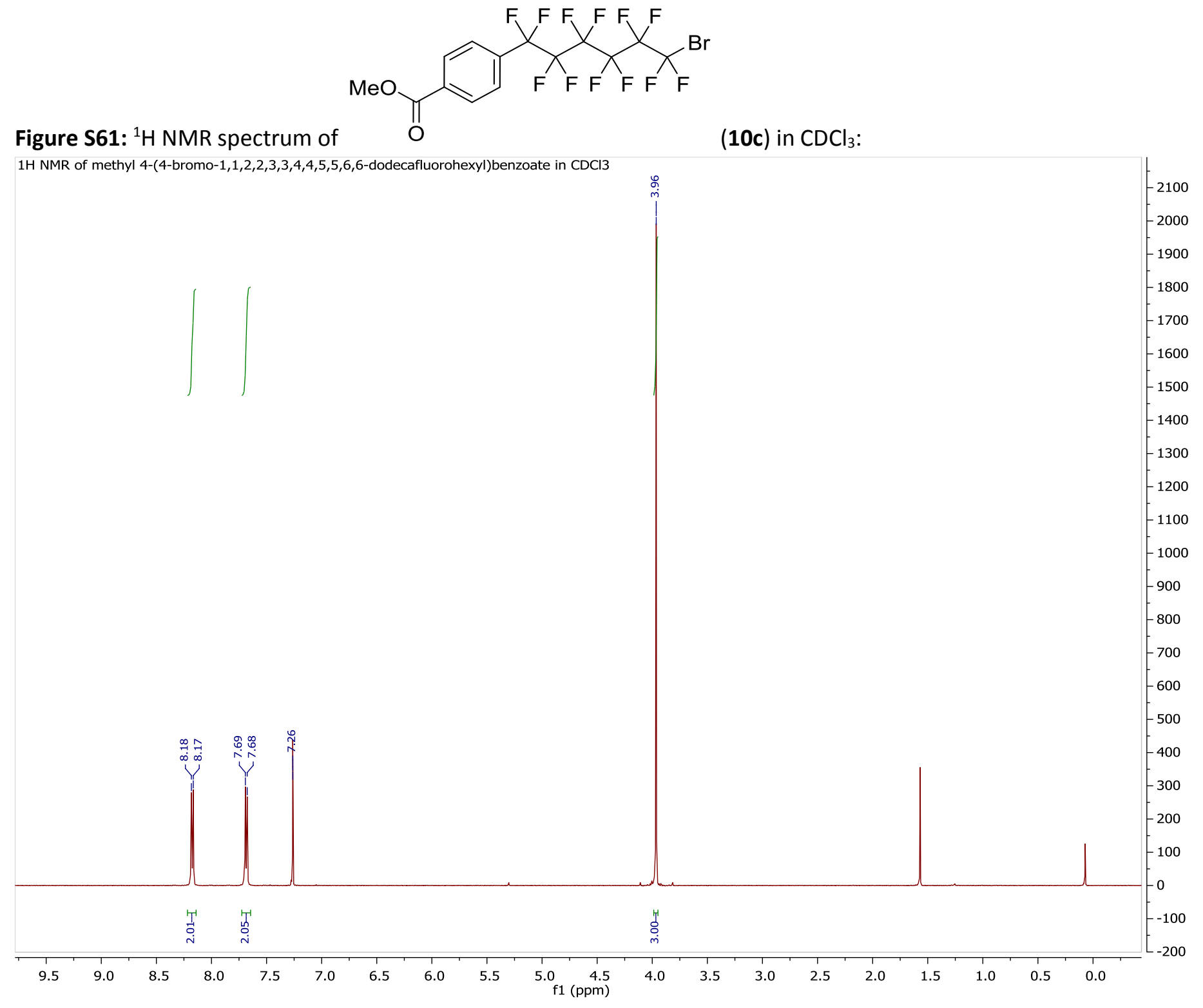


Figure S62: ${ }^{19} \mathrm{~F} N M R$ spectrum of

(10d) in $\mathrm{CDCl}_{3}$ :

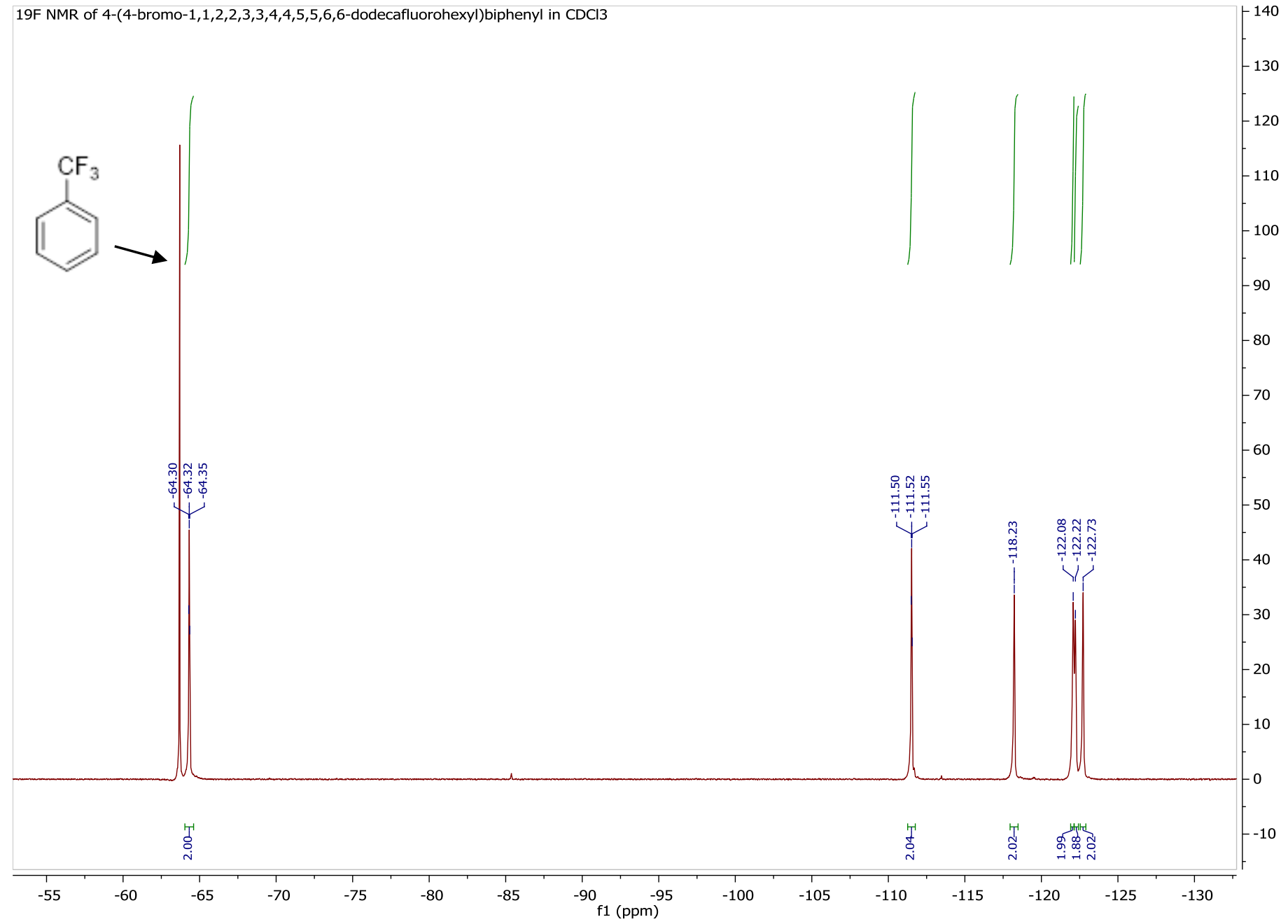


Figure S63: ${ }^{1} \mathrm{H}$ NMR spectrum of

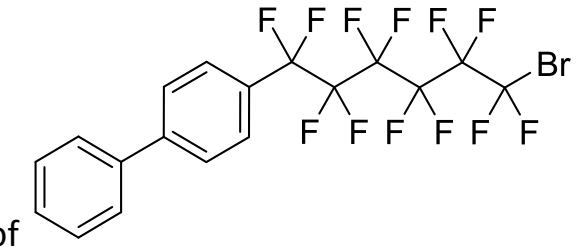

$1 \mathrm{H}$ NMR of 4-(4-bromo-1,1,2,2,3,3,4,4,5,5,6,6-dodecafluorohexyl)biphenyl in $\mathrm{CDCl} 3$

(10d) in $\mathrm{CDCl}_{3}$ :

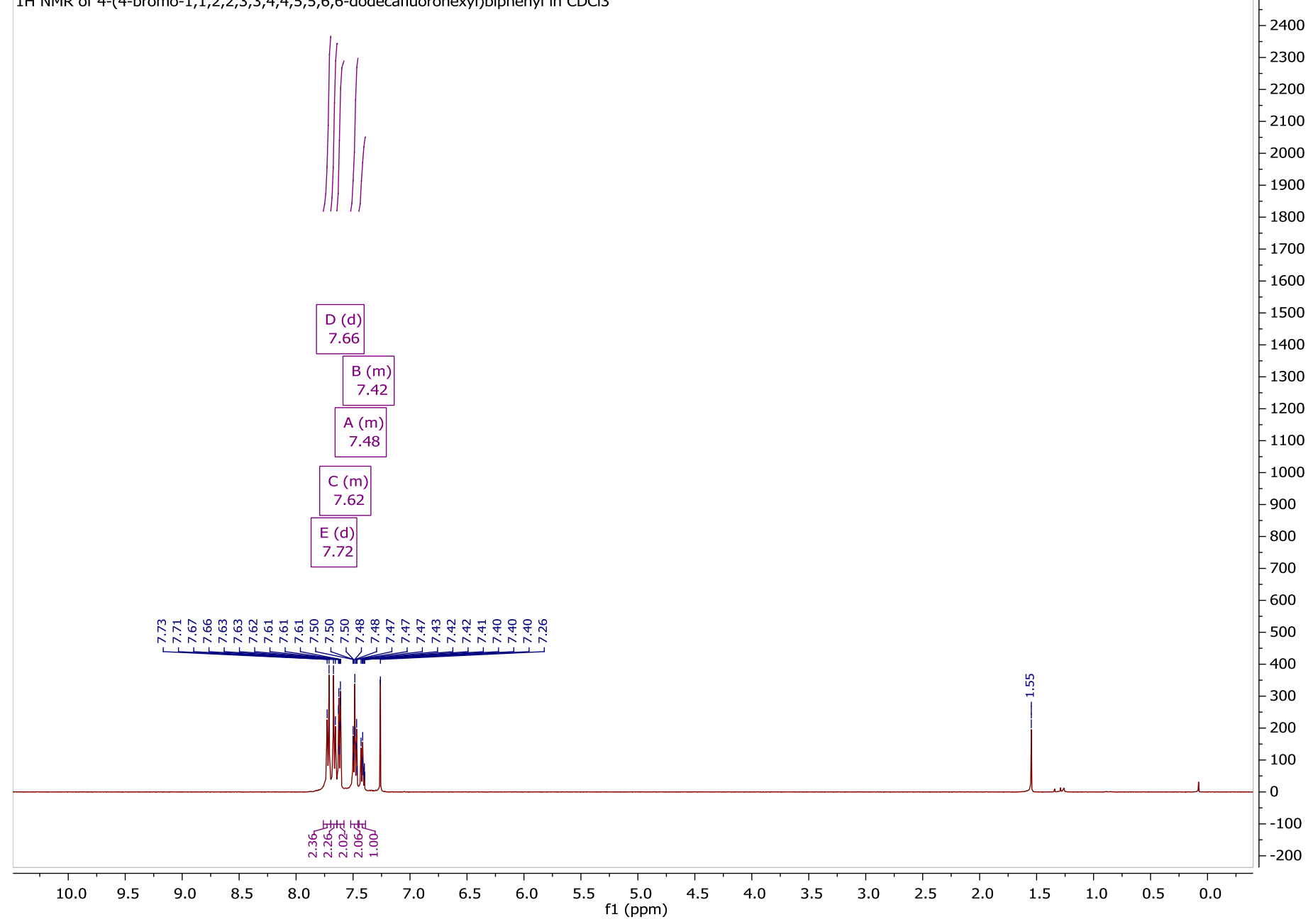

